\section{Pacific Northwest}

National Laboratory

Operated by Battelle for the

U.S. Department of Energy

\title{
1996-2004 Trends in the Single-Family Housing Market: Spatial Analysis of the Residential Sector
}

\author{
D. M. Anderson \\ D. B. Elliott
}

September 2006

Prepared for the U.S. Department of Energy under Contract DE-AC06-76RL01830 


\title{
DISCLAIMER
}

This report was prepared as an account of work sponsored by an agency of the United States Government. Neither the United States Government nor any agency thereof, nor Battelle Memorial Institute, nor any of their employees, makes any warranty, express or implied, or assumes any legal liability or responsibility for the accuracy, completeness, or usefulness of any information, apparatus, product, or process disclosed, or represents that its use would not infringe privately owned rights. Reference herein to any specific commercial product, process, or service by trade name, trademark, manufacturer, or otherwise does not necessarily constitute or imply its endorsement, recommendation, or favoring by the United States Government or any agency thereof, or Battelle Memorial Institute. The views and opinions of authors expressed herein do not necessarily state or reflect those of the United States Government or any agency thereof.

\author{
PACIFIC NORTHWEST NATIONAL LABORATORY \\ operated by \\ BATTELLE \\ for the \\ UNITED STATES DEPARTMENT OF ENERGY \\ under Contract DE-AC06-76RL01830
}

Printed in the United States of America
Available to DOE and DOE contractors from the Office of Scientific and Technical Information, P.O. Box 62, Oak Ridge, TN 37831-0062; ph: (865) 576-8401 fax: (865) 576-5728
email: reports@adonis.osti.gov

\author{
Available to the public from the National Technical Information Service, \\ U.S. Department of Commerce, 5285 Port Royal Rd., Springfield, VA 22161 \\ ph: (800) 553-6847 \\ fax: (703) 605-6900 \\ email: orders@ntis.fedworld.gov \\ online ordering: http://www.ntis.gov/ordering.htm
}




\title{
1996-2004 Trends in the Single-Family Housing Market: Spatial Analysis of the Residential Sector
}

\author{
D. M. Anderson \\ D. B. Elliott
}

September 2006

Prepared for

the U.S. Department of Energy

under Contract DE-AC06-76RL01830

Pacific Northwest National Laboratory

Richland, Washington 99352 


\section{SUMMARY}

This report presents a broad brush analysis of home mortgage and building permit data. It is intended to expose residential sector technology managers to trends in the sector that either cannot be informed by decennial census data, are better informed by considering annual data, or that illustrate spatial sensitivity of a number of important measures. In some cases, results have been presented by climate zone or by Census Region, and potentially could inform existing residential and commercial sector energy models. The trends presented should help inform decisions about how best to pursue energy efficiency with residential home buyers. At a minimum, gaining an understanding of the information here should provide rich background and context for planning technology research in the future.

This report provides a detailed geographic analysis of two specific topics affecting the residential sector. First, we performed an analysis of new construction market trends using annual building permit data. We report summarized tables and national maps to help illustrate market conditions. Second, we performed a detailed geographic analysis of the housing finance market. We analyzed mortgage application data to provide citable statistics and detailed geographic summarization of the residential housing picture in the U.S. for each year in the 1996-2004 period. The databases were linked to geographic information system (GIS) tools to provide various map series detailing the results geographically. Looking at these results geographically may suggest potential new markets for technology development (TD) programs addressing the residential sector that have not been considered previously. For example, we show which lenders affect which regions and which income or mortgage product classes.

These results also highlight the issue of housing affordability. Energy efficiency R\&D programs focused on developing new technology for the residential sector must be conscious of the costs of products resulting from research that will eventually impact the home owner or new home buyer. Results indicate that home values as a proportion of median family income in Building America communities are closely aligned with the national average of home value as a proportion of median income.

We suggest that in a low interest rate environment there is little impediment to continued strength in the single-family housing market. The data on home buyer income suggests that home buyer income growth is outpacing median family income growth, and that the rate of outpacing is increasing during the study period. This would suggest that the national income distribution continues to widen at a steady pace. It also helps explain the continued trend toward an ever-increasing national average home size.

Migration and demographic change are continuing to affect the housing market. Migration to, and population growth within, the Hot-Dry and Hot-Humid climate zones away from the Cold and Mixed-Humid zones - will continue to increase emphasis on efficient cooling technologies and water-saving technologies. Rapid and continued growth in these climate zones is being accompanied by rapid demographic change. Nonwhite minority races and ethnicities are gaining larger shares of the housing market in 
these growing climate zones. This is likely to have cultural implications which technology developers might address with new products or practices.

Mortgage lenders provide one potentially important avenue for moving adoption of energy efficient technology in to the residential sector. If DOE has interests in partnering with lenders to provide special loan products that encourage efficiency adoption, or to lead the financing of Building America developments, it is becoming increasingly important to understand that lender's performance in the home finance market. Lenders are becoming increasingly subject to outside appraisal of their lending practices by Federal examiners, community housing groups, housing activists, interveners, and responsible investor group analysts. To avoid potential embarrassment over practices that some may publicly call questionable, DOE also must be informed of the lending performance of partners in the home lending business.

The interface between urban and rural communities is important for technology research managers to understand in broad context. Sprawling development or otherwise seemingly unplanned development, including the expansion of suburbia or the emergence of exurban development have land use policy implications. The effects on land use can drive local jurisdictions to implement new regulations on growth and development. Trends toward suburban regulation of development have and are likely to continue to affect residential and commercial architecture, community planning (home spacing, green spaces, home/lot size), and consumer behavior (commuting, shopping, recreation, etc.).

Finally, spatial analysis has shown important regional aspects of the residential home buying market. Principally, we suggest and the report bears out that aggregated analysis of the residential sector at the national level involving variables such as income, home value, demography, or climate would tend to hide important regional differences. These differences can manifest themselves at the region, state, or sub-state level or lower in many cases. These differences provide a basis for richer market segmentation analyses, which inform new product development activities. 


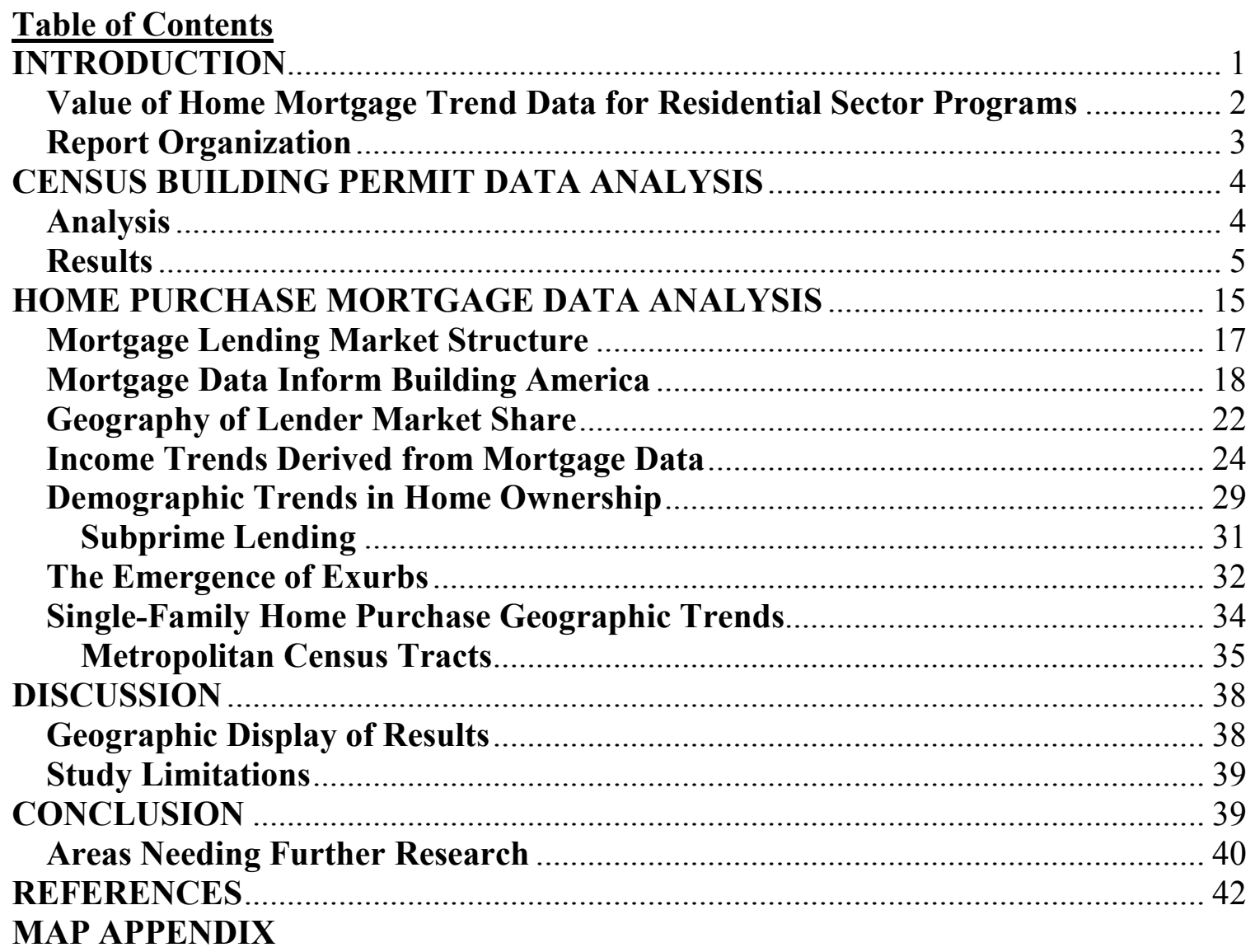

\section{List of Tables}

Table 1. Census Division Unit Summary Statistics .................................................... 5

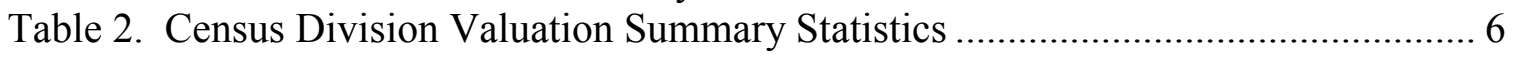

Table 3. Number of Permitted Single Family Units, by Census Division ........................ 8

Table 4. Share of Permitted Single Family Units, by Census Division ........................... 8

Table 5. Valuation of Permitted Single Family Units, by Census Division (Millions of Survey Year Dollars) ............................................................................................ 9

Table 6. Share of Permitted Single Family Unit Valuation, by Census Division............. 9

Table 7. Climate Region Unit Summary Statistics ................................................. 10

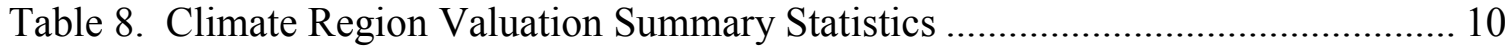

Table 9. Number of Permitted Single Family Units, by Climate Region ....................... 13

Table 10. Share of Permitted Single Family Units, by Climate Region ......................... 13

Table 11. Valuation of Permitted Single Family Units, by Climate Region (Millions of

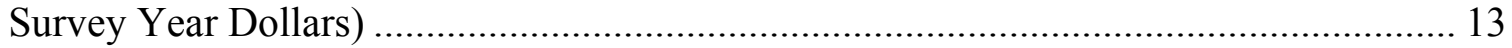

Table 12. Share of Permitted Single Family Unit Valuation, by Climate Region........... 14

Table 13. Dimensions of HMDA-Reported Mortgage Application Data....................... 15

Table 14. Dimensions of HMDA-Reported Originated Loans by Intended Purpose ...... 15

Table 15. Home Purchase Mortgage Activity in Building America Census Tracts (Loans) 
Table 16. Top Home Purchase Mortgage Originators in Building America Census Tracts

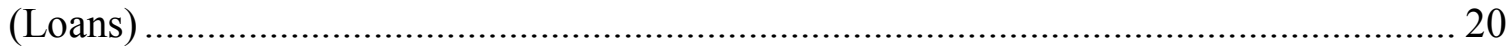

Table 17. 1996-2004 SF Home Purchase Mortgage Volume by Building America

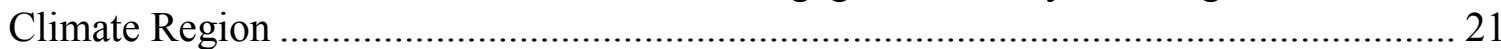

Table 18. 1996-2004 SF Home Purchase Shares by Building America Climate Region 21

Table 19. 2004 Market Share Quartile Constituents 22

Table 20. Building America Census Tracts Single-Family Home Purchase Lending

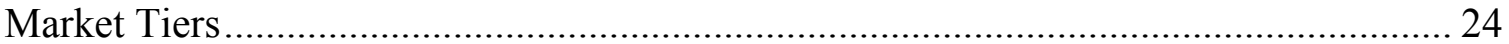

Table 21. 1996-2004 Home Purchase Loans by Borrower Income Class ....................... 25

Table 22. Income Characteristics of Home Purchase Mortgagees ................................. 25

Table 23. Annual Average Borrower Income as a Proportion of HUD Median Family

Income in Building America Census Tracts by Lender.................................................. 28

Table 24. 2004 Home Purchase Proportions by Race and Building America Climate

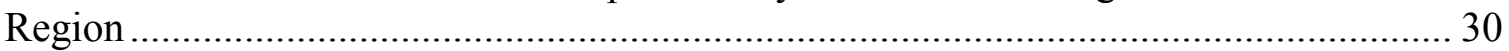

Table 25. 2004 Subprime Home Purchase Loans by Race of Borrower. ......................... 31

Table 26. Exurban Characterization of Significantly Growing Urban Areas ................... 33

\section{List of Figures}

Figure 1. National Average 30-year Conventional Mortgage Rates, 1995-2006 .............. 1

Figure 2. Census Regions and Divisions ................................................................ 7

Figure 3. Building America Adaptation of IECC Climate Zones.................................... 12

Figure 4. 1996-2004 Trends in Mortgage Originations ................................................. 16

Figure 5. 1996-2004 Trends in Mortgage Products ...................................................... 17

Figure 6. 1996-2004 Market Share Trends in the Conventional Home Purchase Mortgage Market...

Figure 7. 1996-2004 Proportion of Home Buying by Building America Climate Region

Figure 8. Trends in Family Income Relative to Home Values ........................................ 26

Figure 9. Income Distribution of Home Buyers in 1996, 2002, and 2004 .................... 27

Figure 10. 1996-2004 Home Values as a Proportion of Median Family Income............ 28

Figure 11. Proportion of Home Purchase Mortgages Originated by Race of Borrower.. 29 Figure 12. Proportion of Home Purchase Mortgages Originated by Race of Borrower in

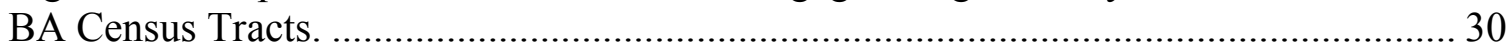




\section{INTRODUCTION}

This report presents a geographic characterization of demographic and home ownership trends present in the residential sector. The motivation for this research stems from the unprecedented expansion of home ownership that has accompanied the significant decline in mortgage interest rates since the mid 1990's. The effect of declining interest rates has lead to sharp decreases in the cost of obtaining and managing a home mortgage that home buyers face. This reduction in cost has made it possible for a large segment of society to qualify for a home mortgage that historically has not had the economic means. It has also increased the level of competition in the mortgage finance industry as the flood of new potential home owners has caused the industry to proliferate into many various financing services and myriad options for home financing that did not exist 10 years ago. As a consequence of these factors, consumers are able to buy more home for the money, refinance a current residence to halve the mortgage term for reduced monthly payments, obtain loans against their home's equity, or take on other forms of consumer debt to finance other aspects of their lives - in place of servicing debt that in decades past was devoted solely to the primary home mortgage.

These rate-induced shifts in home buying power have fueled tremendous expansion of the residential built environment in the period marked by the lower interest rates. This report considers the period 1996-2004 for sake of analysis. Figure 1 illustrates the path of national mortgage interest rates during that period. The intent of this report is to document residential expansion and segmentation geographically. Using the ESRI ArcView geographic information system (GIS), we illustrate with maps many aspects of this expansion that should be of interest to technology researchers in the buildings sector.



Source: Freddie Mac (2006); Y-scale magnified for display purposes

Figure 1. National Average 30-year Conventional Mortgage Rates, 1995-2006 
Specifically, geographic analysis shows the emergence of "exurbs" or emergent islands of civilization featuring large new housing subdivisions that have sprung up beyond traditional suburbia around the outer fringes of large cities. This report also shows the phenomenon of neighborhood gentrification or the infill of established residential neighborhoods with newer, larger, upscale homes sometimes referred to as "mansionization." This report also links demographic trends in society to their effects on the housing market. Home ownership rates among all racial minority groups have grown significantly during the study period, and this report presents broad analysis on this topic. Finally, this report illustrates how using sub-county or census tract data presents a more spatially accurate picture of the residential sector than using county-level, state-level, or national data.

\section{Value of Home Mortgage Trend Data for Residential Sector Programs}

Each year the Federal Financial Institutions Examination Council releases a census of all mortgage finance transactions that occurred at federally regulated institutions in the nation during the calendar year (FFIEC 1997-2005). All institutions (banks, finance companies, mortgage lenders, credit unions, etc.) regulated under the Home Mortgage Disclosure Act (HMDA) must submit a complete census of their qualifying transactions. These microdata include the qualifying income of the home buyer, geographic location of the home to the census tract, the amount financed, the type of financing, and other information about the property, the originating institution, and the borrower. This census of transactions also includes all denied applications for mortgages and provides the summary of denial reasons.

Having microdata on each financed mortgage transaction provides the richest source of annual data available covering the residential sector. However, the richness of the data comes with limitations that must be understood. Because we are talking about mortgage data, the financial values in the data represent amounts financed, not necessarily - in fact rarely - the exact sale prices. Because mortgagees often make down payments as part of obtaining a mortgage (sometimes substantial down payments), mortgage values will be generally a minimum of $10 \%$ less than the sale price of the home. By definition, mortgage data do not include cash transactions, seller-financed transactions, or any other arrangements that did not involve a federally regulated mortgager such as banks, credit unions, mortgage companies, finance companies, certain home building companies, etc. "For sale by owner" transactions are included if the buyer used a federally regulated mortgager to obtain financing. Therefore, although we have an annual census of mortgage transactions covering the vast majority of housing purchases, not all home selling activity is included. Further, although the financial values are mortgage amounts, we made the assumption that generally, trends in amounts financed would mirror trends in home values.

The HMDA data provide the race, income, and sex of each mortgagee. The data also include the bank or other institution making the loan, the census tract of the home, the 
type of financing, whether conventional or government (FHA, VA, etc), and whether the loan application was denied for any reason.

There are several other data elements that would be desirable to include in the HMDA data, but that are not required by HMDA and are not included. Of particular use would be the sales price, square footage, vintage, and the latitude and longitude coordinates of each home. These data are typically captured on mortgage applications, but are not specifically required by HMDA reporters and likely would have privacy issues associated with them.

Regardless of the limitations presented, the HMDA data provide a rich and robust source of very detailed geographic data about the residential sector. With census-tract specificity, the data can be used to characterize large Building America developments around the country and compare those to their surrounding communities. The data can inform a trend analysis of home values based on the trend in mortgage values for any area down to single census tracts. Localized trends in minority home ownership and characterization of underserved housing communities can be identified.

The data also provide the capability to do market share analysis. This is helpful for identifying those lenders that best service specific segments of the residential market. For example, if considering a particular new incentive for home energy efficiency, it might be helpful to understand which lenders best serve the government loan market or the home improvement loan market. Before engaging specific lenders, it would be beneficial to know which lenders have a strong record of performance serving the underserved segments of the housing market (minorities, low-income, economically disadvantaged areas).

The mortgage data also provide the basis for rich spatial analysis of income and home value trends differentiated geographically. In this report we explore the usefulness of these data for identifying exurban development and for neighborhood gentrification. Exurban development, a variant of urban sprawl, continues to be an issue of importance to local governments and their stakeholders in the development industry. An issue associated with neighborhood gentrification that has been gaining increasing media attention recently is the proliferation of "McMansions." This term has been used to refer to both the development of large suburban neighborhoods featuring homogeneous architecture - or to the (re)building of large, lot-filling homes that may be out of character for the older established urban neighborhoods in many cities. We will cover both of these issues in greater detail later in the report.

\section{Report Organization}

This report is organized to provide descriptive material about the methods and data used to develop the geographic and trend analyses in the text. We begin by documenting the analysis of trends in the residential building permits data on home construction and valuation. This is followed by an analysis of the 2000 Census portions dealing with 
home ownership. Next, we present a detailed analysis of home mortgage trends. The report closes with a discussion and conclusions section.

\section{CENSUS BUILDING PERMIT DATA ANALYSIS}

Nationwide, county-level annual building permit data were acquired from the Census Bureau $^{1}$ for the years 1990 through 2004. These survey data included the number of buildings permitted, the number of units permitted, and the associated current-dollar permit valuations. Each type of data was further categorized by the number of units in a building (1, 2, 3-4, and 5+). As this study focuses on single-family housing, only buildings with four or fewer units were considered for analysis.

Unfortunately, not every county surveyed responds in a timely manner each year. In order to provide the most complete and accurate estimates, the Census calculates imputed values for otherwise missing data points. The dataset included both "reported only" values and "estimates with imputation." This analysis employed the latter data. Any data for territories outside the 50 states and the District of Columbia (e.g. Puerto Rico) were excluded.

\section{Analysis}

Given the above-mentioned interest in single-family housing, annual calculations of total units, valuation, and valuation per unit for permitted buildings with four or fewer units formed the basis for the analysis.

The primary analysis period included the years 1996 through 2004. Additionally, the period 2000 through 2004 was examined for comparative purposes. This latter period was chosen for a number of its unique characteristics at least indirectly related to or impacting housing. First, the year 2000 marked the peak and initial subsequent decline of the stock market. Second, January of 2001 saw the Federal Reserve commence down a path of cutting the federal funds rate to its lowest level in over 40 years. Mortgage rates, adjustable-rate mortgage rates in particular, mirrored the federal funds rate decline (see Figure 1). These two factors arguably played a role in the heightened interest in residential real estate during this latter period.

Four key statistics were calculated for each county: total permitted units during the time period of interest; annualized growth rate of permitted units; annualized growth rate of permitted valuation; and annualized growth rate of permitted valuation per unit ${ }^{2}$. With

\footnotetext{
${ }^{1}$ For additional information on Census new residential construction data, see: http://www.census.gov/const/www/newresconstindex.html. For Census building permit data, see: http://www.census.gov/const/www/permitsindex.html.

${ }^{2}$ As noted above, the Census permit data are in current-dollar format. All dollar valuations reported in this section remain in current, rather than constant, dollars. We focused on relative comparisons among counties and other various regions. A real analysis (backing out a national inflation rate) would change absolute numbers and growth rates, but not the relative positions/rankings of the counties, etc. for a given time period. In some limited sense, the permitted-unit growth captured the real portion of growth, since dollars were not an included factor there.
} 
regard to the growth rates of permitted units and valuation - these are growth rates of flows, rather than stocks. In other words, these do not represent the growth of the building stock, but rather the growth in the amount the stock changes each year.

The county-level statistics were linked to spatial data within the ArcGIS 9 geographic information system (GIS). Viewing these data via maps provides the opportunity to see trends and relationships that may be quite difficult to discern from detailed tables.

\section{Results}

Results aggregated to the Census Division and national levels are presented in Tables 1 and 2. Figure 2 provides a map of Census Divisions.

Table 1. Census Division Unit Summary Statistics

\begin{tabular}{|c|c|c|c|c|}
\hline \multirow[b]{2}{*}{$\begin{array}{l}\text { Census } \\
\text { Division }\end{array}$} & \multicolumn{2}{|c|}{ Period Totals } & \multicolumn{2}{|c|}{ Annualized Growth Rates } \\
\hline & $\begin{array}{r}\text { Single Family } \\
\text { Permitted Units } \\
1996-2004 \\
\end{array}$ & $\begin{array}{r}\text { Single Family } \\
\text { Permitted Units } \\
2000-2004 \\
\end{array}$ & $\begin{array}{r}\text { Single Family } \\
\text { Permitted Units } \\
1996-2004 \\
\end{array}$ & $\begin{array}{r}\text { Single Family } \\
\text { Permitted Units } \\
2000-2004 \\
\end{array}$ \\
\hline New England & 371,355 & 212,064 & $3.13 \%$ & $3.62 \%$ \\
\hline Middle Atlantic & 841,007 & 492,440 & $3.52 \%$ & $2.87 \%$ \\
\hline $\begin{array}{l}\text { East North } \\
\text { Central }\end{array}$ & $1,727,018$ & 988,325 & $1.81 \%$ & $3.13 \%$ \\
\hline $\begin{array}{l}\text { West North } \\
\text { Central }\end{array}$ & 776,423 & 457,627 & $3.91 \%$ & $7.64 \%$ \\
\hline South Atlantic & $3,306,500$ & $2,014,115$ & $6.24 \%$ & $9.13 \%$ \\
\hline $\begin{array}{l}\text { East South } \\
\text { Central }\end{array}$ & 656,979 & 376,874 & $3.88 \%$ & $10.03 \%$ \\
\hline $\begin{array}{l}\text { West South } \\
\text { Central }\end{array}$ & $1,359,350$ & 845,047 & $6.90 \%$ & $9.09 \%$ \\
\hline Mountain & $1,486,718$ & 888,529 & $5.99 \%$ & $9.66 \%$ \\
\hline Pacific & $1,537,685$ & 949,584 & $7.19 \%$ & $9.39 \%$ \\
\hline US Total & $12,063,035$ & $7,224,605$ & $5.20 \%$ & $7.75 \%$ \\
\hline
\end{tabular}


Table 2. Census Division Valuation Summary Statistics

\begin{tabular}{|l|r|r|r|r|}
\hline & \multicolumn{4}{|c|}{ Annualized Growth Rates } \\
\cline { 2 - 5 } $\begin{array}{l}\text { Census } \\
\text { Division }\end{array}$ & $\begin{array}{r}\text { Single Family } \\
\text { Permitted } \\
\text { Valuation } \\
\mathbf{1 9 9 6 - \mathbf { 2 0 0 4 }}\end{array}$ & $\begin{array}{r}\text { Single Family } \\
\text { Permitted } \\
\text { Valuation } \\
\mathbf{2 0 0 0 - 2 0 0 4}\end{array}$ & $\begin{array}{r}\text { Single Family } \\
\text { Permitted Value } \\
\text { per Unit } \\
\mathbf{1 9 9 6 - 2 0 0 4}\end{array}$ & $\begin{array}{r}\text { Single Family } \\
\text { Permitted Value } \\
\text { per Unit } \\
\mathbf{2 0 0 0}-\mathbf{2 0 0 4}\end{array}$ \\
\hline New England & $8.79 \%$ & $8.53 \%$ & $5.49 \%$ & $4.75 \%$ \\
\hline Middle Atlantic & $8.62 \%$ & $8.03 \%$ & $4.93 \%$ & $5.01 \%$ \\
\hline $\begin{array}{l}\text { East North } \\
\text { Central }\end{array}$ & $6.61 \%$ & $7.92 \%$ & $4.72 \%$ & $4.65 \%$ \\
\hline $\begin{array}{l}\text { West North } \\
\text { Central }\end{array}$ & $9.13 \%$ & $12.78 \%$ & $5.03 \%$ & $4.78 \%$ \\
\hline South Atlantic & $11.84 \%$ & $14.94 \%$ & $5.27 \%$ & $5.32 \%$ \\
\hline $\begin{array}{l}\text { East South } \\
\text { Central }\end{array}$ & $9.06 \%$ & $15.04 \%$ & $4.99 \%$ & $4.56 \%$ \\
\hline $\begin{array}{l}\text { West South } \\
\text { Central }\end{array}$ & $11.11 \%$ & $11.45 \%$ & $3.94 \%$ & $2.17 \%$ \\
\hline Mountain & $10.84 \%$ & $14.44 \%$ & $4.57 \%$ & $4.36 \%$ \\
\hline Pacific & $12.07 \%$ & $12.48 \%$ & $4.55 \%$ & $2.83 \%$ \\
\hline US Total & $\mathbf{1 0 . 2 7 \%}$ & $\mathbf{1 2 . 3 0 \%}$ & $\mathbf{4 . 8 2 \%}$ & $\mathbf{4 . 2 3 \%}$ \\
\hline
\end{tabular}

For the 1996 through 2004 period, the Pacific Division led all others in the growth rates in both permitted units and valuation. However, Pacific's growth rate of value per unit actually fell below the national mean value. New England held the top spot as measured by that statistic. The South Atlantic Division contained the most permitted units, with over 25 percent of the national total.

On a national level, the 2000 through 2004 period brought significantly higher unit and valuation growth rates. This more rapid growth was widespread, and in some cases quite dramatic. With the exception of the Middle Atlantic Division, all Census Divisions experienced greater unit growth rates. Valuation growth rates increased in all Divisions other than the Middle Atlantic and New England. As for total permitted units, the South Atlantic Division continued to lead the way with slightly more than one quarter of the national figure.

Perhaps the most striking revelation is that the growth rate in value per unit actually slowed, from $4.82 \%$ to $4.23 \%$, for the nation during the 2000 through 2004 period. Only two Divisions experienced even minor increases in these growth rates. However, it is important to note that this result does not imply that home prices grew at a slower rate during this latter period. These valuation numbers only reflect values of structures, and do not include the value of the underlying land. Other research has shown that land is the primary component of home-price appreciation. Davis and Heathcote noted that "the price of residential land grows more quickly but is more volatile than the price of existing homes" (Davis and Heathcote, 2004). 
The annual values for permitted units and valuations by Census Division are presented in Tables 3 and 5, respectively. Corresponding annual percentage shares of national totals are provided in Tables 4 and 6.

While Table 1 shows that the South Atlantic, Pacific, West South Central, and Mountain regions grew much more rapidly in terms of permitted units than the East North Central region, viewing the annual data, and particularly the annual share data, perhaps illustrates this trend more clearly and emphatically. Table 4 shows that East North Central's share of annual national permitted units fell from $16.1 \%$ to $12.4 \%$ in 2004 . During this time period the Pacific, Mountain, and West South Central regions' shares all grew to nearly match or even exceed the East North Central's share of new permits. Table 3 reveals that the Mountain region experienced an especially rapid growth in permitted units between 2002 and 2004, as the annual number of permitted units exploded from about 165 thousand to over 220 thousand.

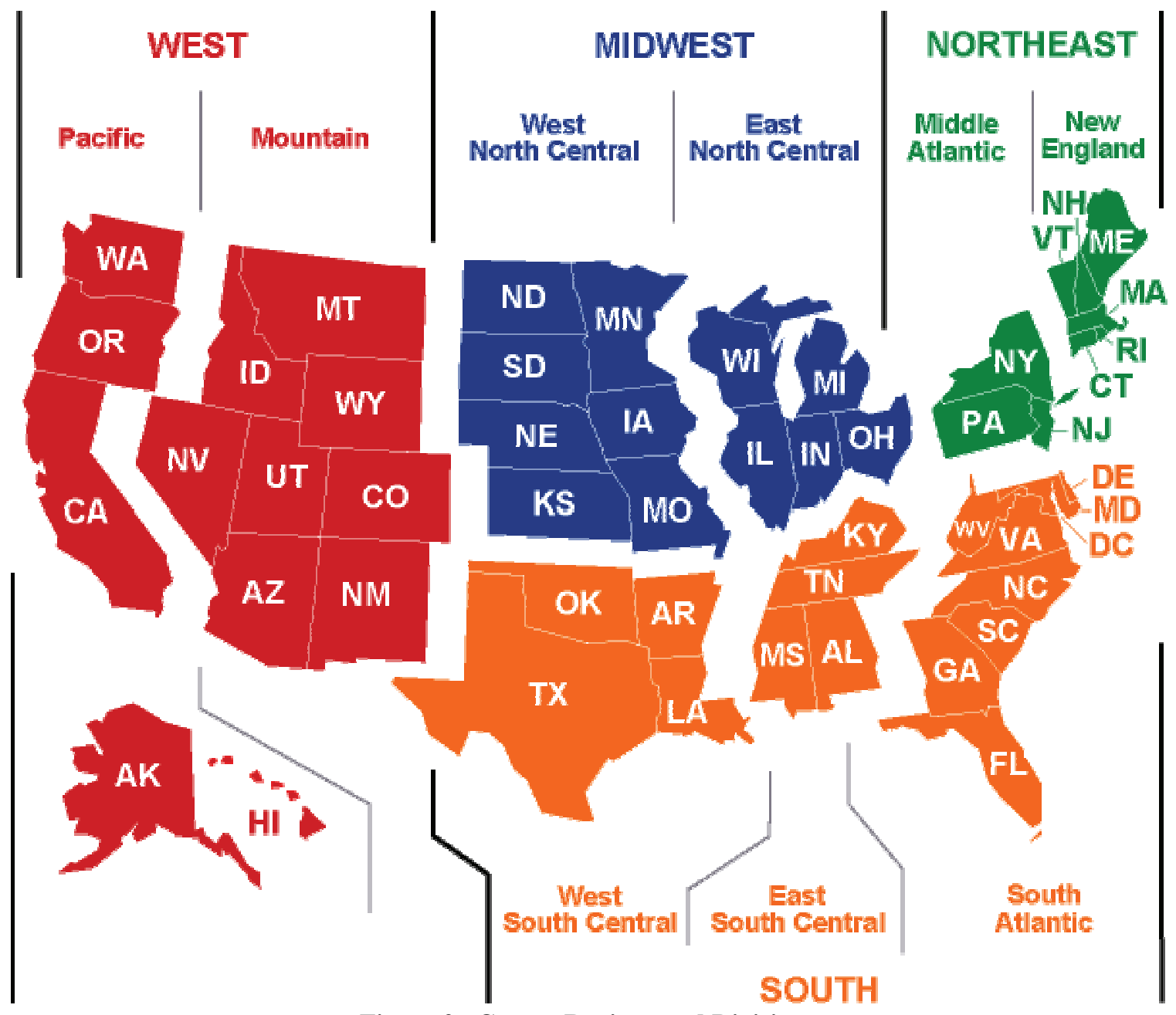

Figure 2. Census Regions and Divisions (http://www.eia.doe.gov/emeu/reps/maps/us_census.html) 
Table 3. Number of Permitted Single Family Units, by Census Division

\begin{tabular}{|l|r|r|r|r|r|r|r|r|r|}
\hline $\begin{array}{l}\text { Census } \\
\text { Division }\end{array}$ & \multicolumn{1}{|c|}{1996} & \multicolumn{1}{c|}{1997} & \multicolumn{1}{c|}{$\mathbf{1 9 9 8}$} & \multicolumn{1}{c|}{$\mathbf{1 9 9 9}$} & $\mathbf{2 0 0 0}$ & $\mathbf{2 0 0 1}$ & $\mathbf{2 0 0 2}$ & $\mathbf{2 0 0 3}$ & $\mathbf{2 0 0 4}$ \\
\hline $\begin{array}{l}\text { New } \\
\text { England }\end{array}$ & 36,554 & 37,429 & 42,608 & 42,700 & 40,575 & 39,258 & 42,730 & 42,732 & 46,769 \\
\hline $\begin{array}{l}\text { Middle } \\
\text { Atlantic }\end{array}$ & 80,217 & 81,958 & 90,900 & 95,492 & 94,426 & 92,163 & 99,984 & 100,109 & 105,758 \\
\hline $\begin{array}{l}\text { East North } \\
\text { Central }\end{array}$ & 182,499 & 171,257 & 187,950 & 196,987 & 186,266 & 189,025 & 194,383 & 207,975 & 210,676 \\
\hline $\begin{array}{l}\text { West North } \\
\text { Central }\end{array}$ & 77,546 & 73,055 & 82,244 & 85,951 & 78,501 & 82,453 & 90,428 & 100,879 & 105,366 \\
\hline $\begin{array}{l}\text { South } \\
\text { Atlantic }\end{array}$ & 299,182 & 302,430 & 335,582 & 355,191 & 342,281 & 363,434 & 392,798 & 430,102 & 485,500 \\
\hline $\begin{array}{l}\text { East South } \\
\text { Central }\end{array}$ & 68,798 & 66,114 & 71,023 & 74,170 & 63,646 & 66,628 & 73,344 & 79,984 & 93,272 \\
\hline $\begin{array}{l}\text { West South } \\
\text { Central }\end{array}$ & 119,376 & 116,177 & 137,649 & 141,101 & 143,731 & 148,425 & 166,247 & 183,117 & 203,527 \\
\hline Mountain & 140,378 & 140,688 & 155,849 & 161,274 & 154,613 & 157,428 & 164,727 & 188,158 & 223,603 \\
\hline Pacific & 131,574 & 142,440 & 153,527 & 160,560 & 160,199 & 164,120 & 185,578 & 210,329 & 229,358 \\
\hline US Total & $\mathbf{1 , 1 3 6 , 1 2 4}$ & $\mathbf{1 , 1 3 1 , 5 4 8}$ & $\mathbf{1 , 2 5 7 , 3 3 2}$ & $\mathbf{1 , 3 1 3 , 4 2 6}$ & $\mathbf{1 , 2 6 4 , 2 3 8}$ & $\mathbf{1 , 3 0 2 , 9 3 4}$ & $\mathbf{1 , 4 1 0 , 2 1 9}$ & $\mathbf{1 , 5 4 3 , 3 8 5}$ & $\mathbf{1 , 7 0 3 , 8 2 9}$ \\
\hline
\end{tabular}

Table 4. Share of Permitted Single Family Units, by Census Division

\begin{tabular}{|l|r|r|r|r|r|r|r|r|r|}
\hline Census Division & $\mathbf{1 9 9 6}$ & $\mathbf{1 9 9 7}$ & $\mathbf{1 9 9 8}$ & $\mathbf{1 9 9 9}$ & $\mathbf{2 0 0 0}$ & $\mathbf{2 0 0 1}$ & $\mathbf{2 0 0 2}$ & $\mathbf{2 0 0 3}$ & $\mathbf{2 0 0 4}$ \\
\hline New England & $3.2 \%$ & $3.3 \%$ & $3.4 \%$ & $3.3 \%$ & $3.2 \%$ & $3.0 \%$ & $3.0 \%$ & $2.8 \%$ & $2.7 \%$ \\
\hline Middle Atlantic & $7.1 \%$ & $7.2 \%$ & $7.2 \%$ & $7.3 \%$ & $7.5 \%$ & $7.1 \%$ & $7.1 \%$ & $6.5 \%$ & $6.2 \%$ \\
\hline East North Central & $16.1 \%$ & $15.1 \%$ & $14.9 \%$ & $15.0 \%$ & $14.7 \%$ & $14.5 \%$ & $13.8 \%$ & $13.5 \%$ & $12.4 \%$ \\
\hline West North Central & $6.8 \%$ & $6.5 \%$ & $6.5 \%$ & $6.5 \%$ & $6.2 \%$ & $6.3 \%$ & $6.4 \%$ & $6.5 \%$ & $6.2 \%$ \\
\hline South Atlantic & $26.3 \%$ & $26.7 \%$ & $26.7 \%$ & $27.0 \%$ & $27.1 \%$ & $27.9 \%$ & $27.9 \%$ & $27.9 \%$ & $28.5 \%$ \\
\hline East South Central & $6.1 \%$ & $5.8 \%$ & $5.6 \%$ & $5.6 \%$ & $5.0 \%$ & $5.1 \%$ & $5.2 \%$ & $5.2 \%$ & $5.5 \%$ \\
\hline West South Central & $10.5 \%$ & $10.3 \%$ & $10.9 \%$ & $10.7 \%$ & $11.4 \%$ & $11.4 \%$ & $11.8 \%$ & $11.9 \%$ & $11.9 \%$ \\
\hline Mountain & $12.4 \%$ & $12.4 \%$ & $12.4 \%$ & $12.3 \%$ & $12.2 \%$ & $12.1 \%$ & $11.7 \%$ & $12.2 \%$ & $13.1 \%$ \\
\hline Pacific & $11.6 \%$ & $12.6 \%$ & $12.2 \%$ & $12.2 \%$ & $12.7 \%$ & $12.6 \%$ & $13.2 \%$ & $13.6 \%$ & $13.5 \%$ \\
\hline US Total & $\mathbf{1 0 0 . 0 \%}$ & $\mathbf{1 0 0 . 0 \%}$ & $\mathbf{1 0 0 . 0 \%}$ & $\mathbf{1 0 0 . 0 \%}$ & $\mathbf{1 0 0 . 0 \%}$ & $\mathbf{1 0 0 . 0 \%}$ & $\mathbf{1 0 0 . 0 \%}$ & $\mathbf{1 0 0 . 0 \%}$ & $\mathbf{1 0 0 . 0 \%}$ \\
\hline
\end{tabular}


Table 5. Valuation of Permitted Single Family Units, by Census Division (Millions of Survey Year Dollars)

\begin{tabular}{|l|c|c|c|c|c|c|c|c|c|}
\hline $\begin{array}{l}\text { Census } \\
\text { Division }\end{array}$ & 1996 & 1997 & 1998 & 1999 & $\mathbf{2 0 0 0}$ & $\mathbf{2 0 0 1}$ & $\mathbf{2 0 0 2}$ & $\mathbf{2 0 0 3}$ & $\mathbf{2 0 0 4}$ \\
\hline New England & $4,244.0$ & $4,532.2$ & $5,385.9$ & $5,763.5$ & $6,002.7$ & $6,133.9$ & $6,855.7$ & $7,073.9$ & $8,329.5$ \\
\hline $\begin{array}{l}\text { Middle } \\
\text { Atlantic }\end{array}$ & $8,050.0$ & $8,511.2$ & $9,927.7$ & $11,029.0$ & $11,452.8$ & $11,590.2$ & $13,183.9$ & $13,936.1$ & $15,599.2$ \\
\hline $\begin{array}{l}\text { East North } \\
\text { Central }\end{array}$ & $20,458.1$ & $19,975.0$ & $22,927.4$ & $25,415.1$ & $25,172.9$ & $26,639.7$ & $28,527.1$ & $31,852.3$ & $34,143.5$ \\
\hline $\begin{array}{l}\text { West North } \\
\text { Central }\end{array}$ & $7,914.8$ & $7,789.2$ & $9,177.5$ & $10,292.8$ & $9,845.3$ & $10,751.1$ & $12,159.0$ & $14,454.3$ & $15,926.7$ \\
\hline South Atlantic & $29,211.8$ & $30,642.1$ & $36,163.1$ & $39,958.5$ & $40,966.9$ & $45,015.4$ & $50,983.1$ & $58,892.2$ & $71,503.6$ \\
\hline $\begin{array}{l}\text { East South } \\
\text { Central }\end{array}$ & $6,138.2$ & $6,220.1$ & $7,091.2$ & $7,866.1$ & $7,012.8$ & $7,558.7$ & $8,601.8$ & $9,820.0$ & $12,282.7$ \\
\hline $\begin{array}{l}\text { West South } \\
\text { Central }\end{array}$ & $11,575.5$ & $11,952.6$ & $14,775.9$ & $16,056.5$ & $17,428.9$ & $17,928.1$ & $20,301.5$ & $23,157.3$ & $26,890.3$ \\
\hline Mountain & $15,216.4$ & $15,516.9$ & $18,059.8$ & $19,787.9$ & $20,209.9$ & $21,070.4$ & $22,932.4$ & $27,487.2$ & $34,666.5$ \\
\hline Pacific & $17,727.9$ & $20,493.6$ & $23,326.1$ & $25,702.8$ & $27,564.1$ & $28,610.0$ & $33,350.3$ & $38,675.1$ & $44,120.6$ \\
\hline US Total & $\mathbf{1 2 0 , 5 3 6 . 7}$ & $\mathbf{1 2 5 , 6 3 3 . 0}$ & $\mathbf{1 4 6 , 8 3 4 . 5}$ & $\mathbf{1 6 1 , 8 7 2 . 1}$ & $\mathbf{1 6 5 , 6 5 6 . 4}$ & $\mathbf{1 7 5 , 2 9 7 . 5}$ & $\mathbf{1 9 6 , 8 9 4 . 8}$ & $\mathbf{2 2 5 , 3 4 8 . 4}$ & $\mathbf{2 6 3 , 4 6 2 . 5}$ \\
\hline
\end{tabular}

Table 6. Share of Permitted Single Family Unit Valuation, by Census Division

\begin{tabular}{|l|r|r|r|r|r|r|r|r|r|}
\hline Census Division & $\mathbf{1 9 9 6}$ & $\mathbf{1 9 9 7}$ & $\mathbf{1 9 9 8}$ & $\mathbf{1 9 9 9}$ & $\mathbf{2 0 0 0}$ & $\mathbf{2 0 0 1}$ & $\mathbf{2 0 0 2}$ & $\mathbf{2 0 0 3}$ & $\mathbf{2 0 0 4}$ \\
\hline New England & $3.5 \%$ & $3.6 \%$ & $3.7 \%$ & $3.6 \%$ & $3.6 \%$ & $3.5 \%$ & $3.5 \%$ & $3.1 \%$ & $3.2 \%$ \\
\hline Middle Atlantic & $6.7 \%$ & $6.8 \%$ & $6.8 \%$ & $6.8 \%$ & $6.9 \%$ & $6.6 \%$ & $6.7 \%$ & $6.2 \%$ & $5.9 \%$ \\
\hline East North Central & $17.0 \%$ & $15.9 \%$ & $15.6 \%$ & $15.7 \%$ & $15.2 \%$ & $15.2 \%$ & $14.5 \%$ & $14.1 \%$ & $13.0 \%$ \\
\hline West North Central & $6.6 \%$ & $6.2 \%$ & $6.3 \%$ & $6.4 \%$ & $5.9 \%$ & $6.1 \%$ & $6.2 \%$ & $6.4 \%$ & $6.0 \%$ \\
\hline South Atlantic & $24.2 \%$ & $24.4 \%$ & $24.6 \%$ & $24.7 \%$ & $24.7 \%$ & $25.7 \%$ & $25.9 \%$ & $26.1 \%$ & $27.1 \%$ \\
\hline East South Central & $5.1 \%$ & $5.0 \%$ & $4.8 \%$ & $4.9 \%$ & $4.2 \%$ & $4.3 \%$ & $4.4 \%$ & $4.4 \%$ & $4.7 \%$ \\
\hline West South Central & $9.6 \%$ & $9.5 \%$ & $10.1 \%$ & $9.9 \%$ & $10.5 \%$ & $10.2 \%$ & $10.3 \%$ & $10.3 \%$ & $10.2 \%$ \\
\hline Mountain & $12.6 \%$ & $12.4 \%$ & $12.3 \%$ & $12.2 \%$ & $12.2 \%$ & $12.0 \%$ & $11.6 \%$ & $12.2 \%$ & $13.2 \%$ \\
\hline Pacific & $14.7 \%$ & $16.3 \%$ & $15.9 \%$ & $15.9 \%$ & $16.6 \%$ & $16.3 \%$ & $16.9 \%$ & $17.2 \%$ & $16.7 \%$ \\
\hline US Total & $\mathbf{1 0 0 . 0 \%}$ & $\mathbf{1 0 0 . 0 \%}$ & $\mathbf{1 0 0 . 0 \%}$ & $\mathbf{1 0 0 . 0 \%}$ & $\mathbf{1 0 0 . 0 \%}$ & $\mathbf{1 0 0 . 0 \%}$ & $\mathbf{1 0 0 . 0} \%$ & $\mathbf{1 0 0 . 0 \%}$ & $\mathbf{1 0 0 . 0 \%}$ \\
\hline
\end{tabular}


It also may be useful to view trend information based on the climate regions adopted by the International Energy Conservation Code (IECC) in 2006. Briggs et al. (2002a, $2002 \mathrm{~b}$ ) provided the geographical classification of IECC climate regions. Results aggregated by climate region are presented in Tables 7 and 8 . Figure 3 provides a map of climate regions.

Table 7. Climate Region Unit Summary Statistics

\begin{tabular}{|l|r|r|r|r|}
\hline \multirow{2}{*}{ Climate Zone } & \multicolumn{2}{|c|}{ Period Totals } & \multicolumn{2}{c|}{ Annualized Growth Rates } \\
\cline { 2 - 5 } & $\begin{array}{r}\text { Single Family } \\
\text { Permitted Units } \\
\mathbf{1 9 9 6 - 2 0 0 4}\end{array}$ & $\begin{array}{r}\text { Single Family } \\
\text { Permitted Units } \\
\mathbf{2 0 0 0 - 2 0 0 4}\end{array}$ & $\begin{array}{r}\text { Single Family } \\
\text { Permitted Units } \\
\mathbf{1 9 9 6 - 2 0 0 4}\end{array}$ & $\begin{array}{r}\text { Single Family } \\
\text { Permitted Units } \\
\mathbf{2 0 0 0} \mathbf{2 0 0 4}\end{array}$ \\
\hline Hot-Humid & $2,010,615$ & $1,277,382$ & $8.45 \%$ & $12.61 \%$ \\
\hline Hot-Dry & $1,587,787$ & $1,016,500$ & $9.69 \%$ & $12.44 \%$ \\
\hline Marine & 542,679 & 294,682 & $1.44 \%$ & $3.97 \%$ \\
\hline Mixed-Humid & $4,038,017$ & $2,377,345$ & $4.39 \%$ & $6.70 \%$ \\
\hline Mixed-Dry & 102,277 & 64,490 & $6.99 \%$ & $11.10 \%$ \\
\hline Cold & $3,633,594$ & $2,108,291$ & $2.99 \%$ & $4.42 \%$ \\
\hline Very Cold & 122,778 & 72,873 & $3.96 \%$ & $5.83 \%$ \\
\hline Subarctic & 2,623 & 1,321 & $2.30 \%$ & $19.16 \%$ \\
\hline Not Assignable & 22,665 & 11,721 & $-4.45 \%$ & $-2.23 \%$ \\
\hline US Total & $\mathbf{1 2 , 0 6 3 , 0 3 5}$ & $\mathbf{7 , 2 2 4 , 6 0 5}$ & $\mathbf{5 . 2 0 \%}$ & $\mathbf{7 . 7 5 \%}$ \\
\hline
\end{tabular}

Table 8. Climate Region Valuation Summary Statistics

\begin{tabular}{|c|c|c|c|c|}
\hline \multirow[b]{2}{*}{ Climate Zone } & \multicolumn{4}{|c|}{ Annualized Growth Rates } \\
\hline & $\begin{array}{r}\text { Single Family } \\
\text { Permitted } \\
\text { Valuation } \\
1996-2004\end{array}$ & $\begin{array}{r}\text { Single Family } \\
\text { Permitted } \\
\text { Valuation } \\
2000-2004\end{array}$ & $\begin{array}{r}\text { Single Family } \\
\text { Permitted Value } \\
\text { per Unit } \\
1996-2004\end{array}$ & $\begin{array}{r}\text { Single Family } \\
\text { Permitted Value } \\
\text { per Unit } \\
2000-2004\end{array}$ \\
\hline Hot-Humid & $14.06 \%$ & $17.57 \%$ & $5.17 \%$ & $4.40 \%$ \\
\hline Hot-Dry & $14.30 \%$ & $15.50 \%$ & $4.20 \%$ & $2.72 \%$ \\
\hline Marine & $6.80 \%$ & $8.87 \%$ & $5.28 \%$ & $4.71 \%$ \\
\hline Mixed-Humid & $9.37 \%$ & $11.27 \%$ & $4.77 \%$ & $4.29 \%$ \\
\hline Mixed-Dry & $10.14 \%$ & $16.47 \%$ & $2.94 \%$ & $4.84 \%$ \\
\hline Cold & $8.19 \%$ & $9.62 \%$ & $5.05 \%$ & $4.97 \%$ \\
\hline Very Cold & $9.37 \%$ & $9.17 \%$ & $5.21 \%$ & $3.15 \%$ \\
\hline Subarctic & $6.85 \%$ & $26.12 \%$ & $4.45 \%$ & $5.84 \%$ \\
\hline Not Assignable 3 & $3.12 \%$ & $-1.60 \%$ & $7.92 \%$ & $0.65 \%$ \\
\hline US Total & $10.27 \%$ & $12.30 \%$ & $4.82 \%$ & $4.23 \%$ \\
\hline
\end{tabular}

For the 1996 through 2004 period, the hot-dry region, closely followed by hot-humid, led all others in the growth rates in permitted units and valuation. The mixed-dry and mixedhumid regions ranked third and fourth in each of these categories, respectively. Together, these four regions accounted for over 60 percent of permitted units during the period.

\footnotetext{
${ }^{3}$ Some Census permit data were not assignable to distinct climate zones. These data were not associated with a particular county, but rather with a "balance of state" designation used by the Census in some cases. For states with only one climate zone, balance of state data could be assigned to a unique climate zone. However, in each of these cases the states in question contained multiple climate zones. As a result, these data were placed in a "not assignable" climate zone category.
} 
Most of the rest fell into the cold region. The marine, hot-humid, and cold regions led in growth rate of value per unit, at slightly over 5 percent for each region.

For the 2000 through 2004 time period, the subarctic region led all others in the growth rates in permitted units, valuation, and valuation per unit. The total number of units permitted in this region was quite small, however, at 2,623. As for regions with more significant numbers of permitted units, hot-humid, hot-dry, and mixed-dry ranked one through three in growth of permitted units, respectively. The same regions held the top three spots for valuation growth, although hot-dry and mixed-dry traded spots. Every region experienced higher growth rates in permitted units, and all except the very cold region saw higher growth rates in valuation. The growth rates of value per unit decreased in all regions other than mixed-dry and subarctic during this period. The annual values for permitted units and valuations by climate region are presented in Tables 9 and 11, respectively. Corresponding annual percentage shares of national totals are provided in Tables 10 and 12 .

Table 7 shows that the Hot-Humid and Hot-Dry climate zones grew much more rapidly than the Cold zone during the analysis period. Table 10's share data provide a more detailed view of that shift. Between 1996 and 2004, the Hot-Humid zone's share of permitted units grew from $15.2 \%$ to $19.3 \%$, and the Hot-Dry share grew from $10.9 \%$ to $15.3 \%$. These areas gained primarily at the expense of the Cold zone, which saw its share fall from $32.4 \%$ to $27.3 \%$.

Maps of county-level results are provided in the map appendix as Maps A.1 through A.8. For each of the two time periods discussed in this section, these maps provide visual representations of units permitted, as well as annualized growth rates for units permitted, permit valuation, and permitted value per unit.

While a very large number of single family units continue to be built in the northern part of the country, it is clear that during the analysis period, building growth measurably shifted from colder climates in the north to hotter climates in the south. The maps assist in providing a more finely detailed view than easily provided by the tabular data discussed to this point. In particular, maps A.7 and A.8 reveal that Florida, parts of Texas, Nevada, Arizona, and Southern California grew much more rapidly than older population centers in the north. 


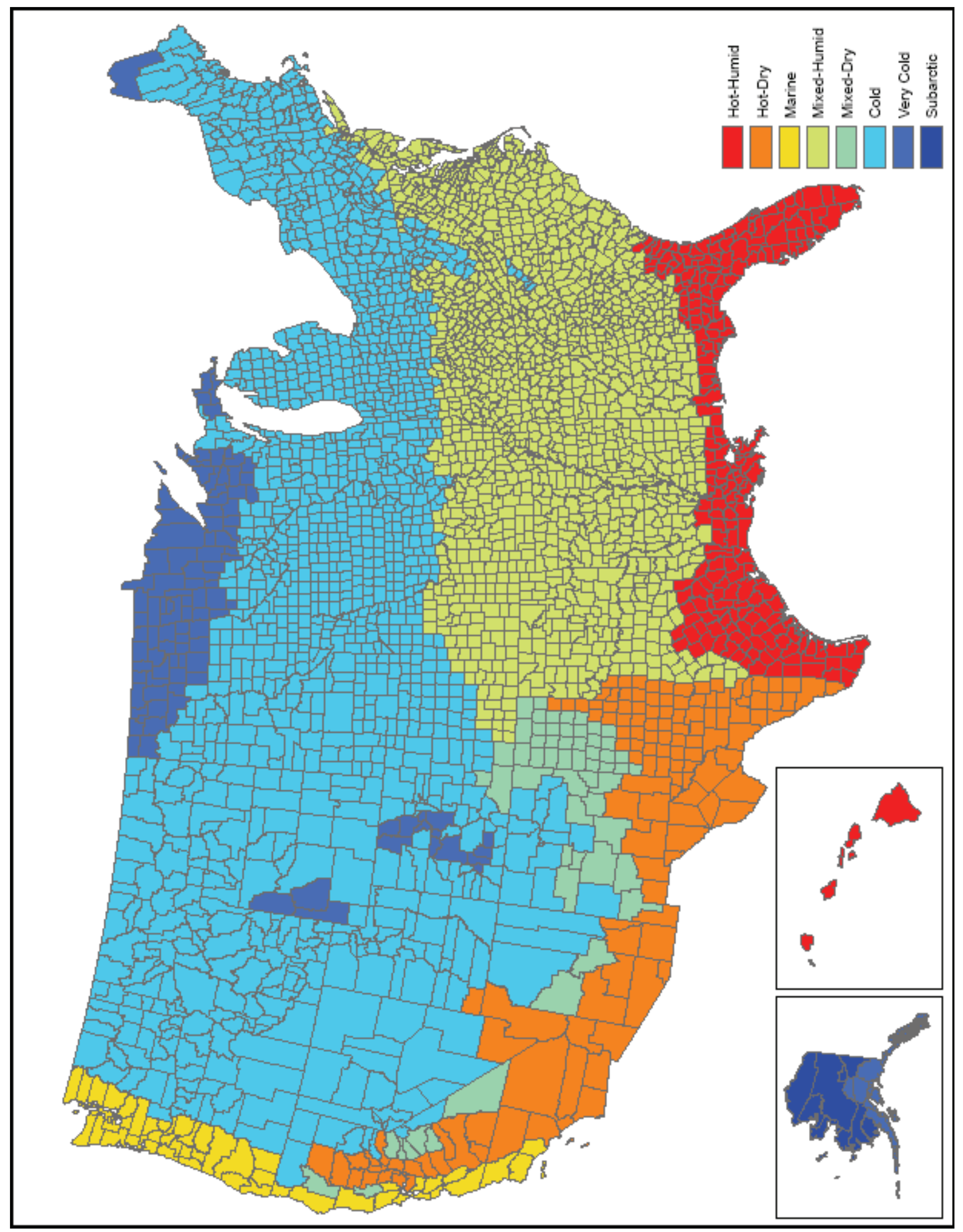

Figure 3. Building America Adaptation of IECC Climate Zones 
Table 9. Number of Permitted Single Family Units, by Climate Region

\begin{tabular}{|l|r|r|r|r|r|r|r|r|r|}
\hline $\begin{array}{l}\text { Climate } \\
\text { Zone }\end{array}$ & $\mathbf{1 9 9 6}$ & $\mathbf{1 9 9 7}$ & $\mathbf{1 9 9 8}$ & $\mathbf{1 9 9 9}$ & $\mathbf{2 0 0 0}$ & $\mathbf{2 0 0 1}$ & $\mathbf{2 0 0 2}$ & $\mathbf{2 0 0 3}$ & $\mathbf{2 0 0 4}$ \\
\hline Hot-Humid & 172,220 & 168,851 & 190,340 & 201,822 & 204,893 & 218,095 & 241,783 & 283,101 & 329,510 \\
\hline Hot-Dry & 124,238 & 135,265 & 150,602 & 161,182 & 162,889 & 172,853 & 192,181 & 228,173 & 260,404 \\
\hline Marine & 58,806 & 62,224 & 64,104 & 62,863 & 56,413 & 53,388 & 57,398 & 61,553 & 65,930 \\
\hline $\begin{array}{l}\text { Mixed- } \\
\text { Humid }\end{array}$ & 388,599 & 388,851 & 432,698 & 450,524 & 422,720 & 436,646 & 473,157 & 496,924 & 547,898 \\
\hline Mixed-Dry & 9,233 & 8,473 & 9,528 & 10,553 & 10,407 & 10,686 & 12,812 & 14,729 & 15,856 \\
\hline Cold & 367,943 & 353,534 & 392,704 & 411,122 & 391,597 & 395,143 & 415,708 & 440,239 & 465,604 \\
\hline Very Cold & 11,934 & 11,449 & 13,774 & 12,748 & 12,975 & 13,523 & 14,389 & 15,708 & 16,278 \\
\hline Subarctic & 316 & 366 & 305 & 315 & 188 & 217 & 244 & 293 & 379 \\
\hline $\begin{array}{l}\text { Not } \\
\text { Assignable }\end{array}$ & 2,835 & 2,535 & 3,277 & 2,297 & 2,156 & 2,383 & 2,547 & 2,665 & 1,970 \\
\hline US Total & $1,136,124$ & $1,131,548$ & $1,257,332$ & $1,313,426$ & $1,264,238$ & $1,302,934$ & $1,410,219$ & $1,543,385$ & $1,703,829$ \\
\hline
\end{tabular}

Table 10. Share of Permitted Single Family Units, by Climate Region

\begin{tabular}{|l|r|r|r|r|r|r|r|r|r|}
\hline Climate Zone & $\mathbf{1 9 9 6}$ & $\mathbf{1 9 9 7}$ & $\mathbf{1 9 9 8}$ & $\mathbf{1 9 9 9}$ & $\mathbf{2 0 0 0}$ & $\mathbf{2 0 0 1}$ & $\mathbf{2 0 0 2}$ & $\mathbf{2 0 0 3}$ & $\mathbf{2 0 0 4}$ \\
\hline Hot-Humid & $15.2 \%$ & $14.9 \%$ & $15.1 \%$ & $15.4 \%$ & $16.2 \%$ & $16.7 \%$ & $17.1 \%$ & $18.3 \%$ & $19.3 \%$ \\
\hline Hot-Dry & $10.9 \%$ & $12.0 \%$ & $12.0 \%$ & $12.3 \%$ & $12.9 \%$ & $13.3 \%$ & $13.6 \%$ & $14.8 \%$ & $15.3 \%$ \\
\hline Marine & $5.2 \%$ & $5.5 \%$ & $5.1 \%$ & $4.8 \%$ & $4.5 \%$ & $4.1 \%$ & $4.1 \%$ & $4.0 \%$ & $3.9 \%$ \\
\hline Mixed-Humid & $34.2 \%$ & $34.4 \%$ & $34.4 \%$ & $34.3 \%$ & $33.4 \%$ & $33.5 \%$ & $33.6 \%$ & $32.2 \%$ & $32.2 \%$ \\
\hline Mixed-Dry & $0.8 \%$ & $0.7 \%$ & $0.8 \%$ & $0.8 \%$ & $0.8 \%$ & $0.8 \%$ & $0.9 \%$ & $1.0 \%$ & $0.9 \%$ \\
\hline Cold & $32.4 \%$ & $31.2 \%$ & $31.2 \%$ & $31.3 \%$ & $31.0 \%$ & $30.3 \%$ & $29.5 \%$ & $28.5 \%$ & $27.3 \%$ \\
\hline Very Cold & $1.1 \%$ & $1.0 \%$ & $1.1 \%$ & $1.0 \%$ & $1.0 \%$ & $1.0 \%$ & $1.0 \%$ & $1.0 \%$ & $1.0 \%$ \\
\hline Subarctic & $0.0 \%$ & $0.0 \%$ & $0.0 \%$ & $0.0 \%$ & $0.0 \%$ & $0.0 \%$ & $0.0 \%$ & $0.0 \%$ & $0.0 \%$ \\
\hline Not Assignable 3 & $0.2 \%$ & $0.2 \%$ & $0.3 \%$ & $0.2 \%$ & $0.2 \%$ & $0.2 \%$ & $0.2 \%$ & $0.2 \%$ & $0.1 \%$ \\
\hline US Total & $\mathbf{1 0 0 . 0 \%}$ & $\mathbf{1 0 0 . 0 \%}$ & $\mathbf{1 0 0 . 0 \%}$ & $\mathbf{1 0 0 . 0} \%$ & $\mathbf{1 0 0 . 0} \%$ & $\mathbf{1 0 0 . 0} \%$ & $\mathbf{1 0 0 . 0 \%}$ & $\mathbf{1 0 0 . 0} \%$ & $\mathbf{1 0 0 . 0} \%$ \\
\hline
\end{tabular}

Table 11. Valuation of Permitted Single Family Units, by Climate Region (Millions of Survey Year Dollars)

\begin{tabular}{|l|r|r|r|r|r|r|r|r|r|}
\hline $\begin{array}{l}\text { Climate } \\
\text { Zone }\end{array}$ & $\mathbf{1 9 9 6}$ & $\mathbf{1 9 9 7}$ & $\mathbf{1 9 9 8}$ & $\mathbf{1 9 9 9}$ & $\mathbf{2 0 0 0}$ & $\mathbf{2 0 0 1}$ & $\mathbf{2 0 0 2}$ & $\mathbf{2 0 0 3}$ & $\mathbf{2 0 0 4}$ \\
\hline Hot-Humid & $16,492.1$ & $16,906.2$ & $20,025.5$ & $22,432.4$ & $24,724.5$ & $26,776.9$ & $30,825.1$ & $38,253.0$ & $47,235.6$ \\
\hline Hot-Dry & $14,995.0$ & $17,102.5$ & $19,823.2$ & $22,994.6$ & $24,541.2$ & $26,529.6$ & $30,366.9$ & $36,761.2$ & $43,680.1$ \\
\hline Marine & $7,699.8$ & $8,672.3$ & $9,411.5$ & $9,275.8$ & $9,277.5$ & $9,224.8$ & $10,428.0$ & $11,661.2$ & $13,031.8$ \\
\hline $\begin{array}{l}\text { Mixed- } \\
\text { Humid }\end{array}$ & $37,950.0$ & $39,713.6$ & $46,834.3$ & $51,345.2$ & $50,669.3$ & $53,775.4$ & $60,404.2$ & $66,116.1$ & $77,677.2$ \\
\hline Mixed-Dry & $1,194.0$ & 962.2 & $1,096.0$ & $1,313.4$ & $1,404.7$ & $1,475.8$ & $1,882.9$ & $2,209.4$ & $2,585.3$ \\
\hline Cold & $40,620.3$ & $40,654.2$ & $47,598.4$ & $52,451.6$ & $52,799.4$ & $55,245.3$ & $60,456.0$ & $67,520.5$ & $76,229.5$ \\
\hline Very Cold & $1,278.2$ & $1,307.9$ & $1,640.9$ & $1,697.6$ & $1,842.5$ & $1,853.8$ & $2,061.2$ & $2,327.7$ & $2,616.8$ \\
\hline Subarctic & 31.5 & 40.4 & 30.4 & 31.0 & 21.2 & 26.4 & 31.0 & 44.4 & 53.5 \\
\hline $\begin{array}{l}\text { Not } \\
\text { Assignable }\end{array}$ & 275.8 & 273.6 & 374.4 & 330.3 & 376.1 & 389.5 & 439.6 & 454.9 & 352.7 \\
\hline & $120,536$. & & & & & & & & \\
US Total & 7 & $125,633.0$ & $146,834.5$ & $161,872.1$ & $165,656.4$ & $175,297.5$ & $196,894.8$ & $225,348.4$ & $263,462.5$ \\
\hline
\end{tabular}


Table 12. Share of Permitted Single Family Unit Valuation, by Climate Region

\begin{tabular}{|l|r|r|r|r|r|r|r|r|r|}
\hline Climate Zone & $\mathbf{1 9 9 6}$ & $\mathbf{1 9 9 7}$ & $\mathbf{1 9 9 8}$ & $\mathbf{1 9 9 9}$ & $\mathbf{2 0 0 0}$ & $\mathbf{2 0 0 1}$ & $\mathbf{2 0 0 2}$ & $\mathbf{2 0 0 3}$ & $\mathbf{2 0 0 4}$ \\
\hline Hot-Humid & $13.7 \%$ & $13.5 \%$ & $13.6 \%$ & $13.9 \%$ & $14.9 \%$ & $15.3 \%$ & $15.7 \%$ & $17.0 \%$ & $17.9 \%$ \\
\hline Hot-Dry & $12.4 \%$ & $13.6 \%$ & $13.5 \%$ & $14.2 \%$ & $14.8 \%$ & $15.1 \%$ & $15.4 \%$ & $16.3 \%$ & $16.6 \%$ \\
\hline Marine & $6.4 \%$ & $6.9 \%$ & $6.4 \%$ & $5.7 \%$ & $5.6 \%$ & $5.3 \%$ & $5.3 \%$ & $5.2 \%$ & $4.9 \%$ \\
\hline Mixed-Humid & $31.5 \%$ & $31.6 \%$ & $31.9 \%$ & $31.7 \%$ & $30.6 \%$ & $30.7 \%$ & $30.7 \%$ & $29.3 \%$ & $29.5 \%$ \\
\hline Mixed-Dry & $1.0 \%$ & $0.8 \%$ & $0.7 \%$ & $0.8 \%$ & $0.8 \%$ & $0.8 \%$ & $1.0 \%$ & $1.0 \%$ & $1.0 \%$ \\
\hline Cold & $33.7 \%$ & $32.4 \%$ & $32.4 \%$ & $32.4 \%$ & $31.9 \%$ & $31.5 \%$ & $30.7 \%$ & $30.0 \%$ & $28.9 \%$ \\
\hline Very Cold & $1.1 \%$ & $1.0 \%$ & $1.1 \%$ & $1.0 \%$ & $1.1 \%$ & $1.1 \%$ & $1.0 \%$ & $1.0 \%$ & $1.0 \%$ \\
\hline Subarctic & $0.0 \%$ & $0.0 \%$ & $0.0 \%$ & $0.0 \%$ & $0.0 \%$ & $0.0 \%$ & $0.0 \%$ & $0.0 \%$ & $0.0 \%$ \\
\hline Not Assignable & $0.2 \%$ & $0.2 \%$ & $0.3 \%$ & $0.2 \%$ & $0.2 \%$ & $0.2 \%$ & $0.2 \%$ & $0.2 \%$ & $0.1 \%$ \\
\hline US Total $^{3}$ & $\mathbf{1 0 0 . 0 \%}$ & $\mathbf{1 0 0 . 0 \%}$ & $\mathbf{1 0 0 . 0 \%}$ & $\mathbf{1 0 0 . 0} \%$ & $\mathbf{1 0 0 . 0} \%$ & $\mathbf{1 0 0 . 0} \%$ & $\mathbf{1 0 0 . 0 \%}$ & $\mathbf{1 0 0 . 0 \%}$ & $\mathbf{1 0 0 . 0} \%$ \\
\hline
\end{tabular}




\section{HOME PURCHASE MORTGAGE DATA ANALYSIS}

The building permit data, by definition, covers new home construction. Home purchase mortgage data covers the financing of both new and existing homes. As introduced earlier, we developed the analysis of trends in the residential sector using data published by the Federal Financial Institutions Examination Council (FFIEC 1997-2005). Specifically, we utilized just the data on home purchase mortgage originations. These data required extraction from the full FFIEC data sets to distinguish these types of mortgages from home refinances, home improvement loans, loans purchased by banks for investment purposes, and all denied or withdrawn mortgage applications. Table 13 illustrates the dimensions of the annual HMDA-required reported mortgage applications data.

Table 13. Dimensions of HMDA-Reported Mortgage Application Data

\begin{tabular}{|c|c|c|c|c|c|c|c|c|c|}
\hline Application Disposition & 1996 & 1997 & 1998 & 1999 & 2000 & 2001 & 2002 & 2003 & 2004 \\
\hline Originated & $7,465,915$ & $7,939,432$ & $12,207,047$ & $10,227,513$ & $8,138,192$ & $13,671,112$ & $16,112,062$ & $21,420,330$ & $15,028,550$ \\
\hline Approved, Not Accepted & $1,006,606$ & $1,358,519$ & $1,860,875$ & $2,010,202$ & $1,669,853$ & $1,882,482$ & $2,232,099$ & $2,736,941$ & $2,387,464$ \\
\hline Denied & $3,090,083$ & $3,758,900$ & $4,822,068$ & $4,964,100$ & $4,723,956$ & $5,092,040$ & $4,545,202$ & $5,830,700$ & $6,035,717$ \\
\hline Withdrawn & $1,163,855$ & $1,212,525$ & $1,968,253$ & $2,125,128$ & $1,799,785$ & $2,572,290$ & $2,735,555$ & $3,554,604$ & $3,612,831$ \\
\hline Incomplete & 205,367 & 279,072 & 457,806 & 578,924 & 503,752 & 603,714 & 815,973 & 779,496 & $1,064,503$ \\
\hline Purchased & $1,792,967$ & $2,143,517$ & $3,219,264$ & $3,005,492$ & $2,397,111$ & $3,755,395$ & $4,794,967$ & $7,234,705$ & $5,146,617$ \\
\hline Pre-Approval Denied & - & - & - & - & - & - & - & - & 165,186 \\
\hline Pre-Approval Not Accepted & - & - & - & - & - & - & - & - & 166,868 \\
\hline Miscoded/Unknown & 242 & - & - & 420 & 526 & 1,454 & 182 & 88 & - \\
\hline Total Applications Reported & $14,725,035$ & $16,691,965$ & $24,535,313$ & $22,911,779$ & $19,233,175$ & $27,578,487$ & $31,236,040$ & $41,556,864$ & $33,607,736$ \\
\hline
\end{tabular}

For this report, we are especially interested in the information content available from the single-family (SF) home purchase loans that actually were originated by the reporting institutions. These represent successful home buyers. These are a subset of the originated applications that appear in Table 13. Table 14 indicates the dimensions of this subset of loans by the principal purpose for the loan.

Table 14. Dimensions of HMDA-Reported Originated Loans by Intended Purpose

\begin{tabular}{|l|r|r|r|r|r|r|r|r|r|}
\hline Loan Purpose & \multicolumn{1}{|c|}{1996} & \multicolumn{1}{c}{1997} & \multicolumn{1}{c}{1998} & \multicolumn{1}{c}{1999} & \multicolumn{1}{c}{2000} & \multicolumn{1}{c}{2001} & \multicolumn{1}{c}{2002} & \multicolumn{1}{c}{2003} & 2004 \\
\hline Home Purchase & $3,789,219$ & $4,039,559$ & $4,528,525$ & $4,849,772$ & $4,783,183$ & $4,932,840$ & $5,095,867$ & $5,574,752$ & $6,452,860$ \\
\hline Home Improvement & $1,086,362$ & $1,024,152$ & 967,978 & 956,996 & 893,097 & 825,098 & 717,845 & 679,463 & 969,479 \\
\hline Mortgage Refinance & $2,567,071$ & $2,849,098$ & $6,676,193$ & $4,386,234$ & $2,433,619$ & $7,877,379$ & $10,256,766$ & $15,117,722$ & $7,606,211$ \\
\hline Multifamily & 23,263 & 26,623 & 34,351 & 34,511 & 27,846 & 35,563 & 41,465 & 48,379 & \\
\hline Total Loans & $7,465,915$ & $7,939,432$ & $12,207,047$ & $10,227,513$ & $8,137,745$ & $13,670,880$ & $16,111,943$ & $21,420,316$ & $15,028,550$ \\
\hline
\end{tabular}

Note: Multifamily loans were dropped as a HMDA requirement in 2004.

Two guiding observations from these data are possible. First, as would be expected in a climate of falling interest rates, SF home purchases are rising steadily over time. Second, interest rate movements also cause volatility in the refinance market. There can be large swings in year-over-year volumes of mortgage refinances. Figure 4 illustrates this point more clearly. Two clear "refinance booms" are discernable during the study period. The first peaks in 1998, and the second, more pronounced boom peaks in 2003. Notice that these booms correspond very closely with the path of interest rates shown in Figure 1. 


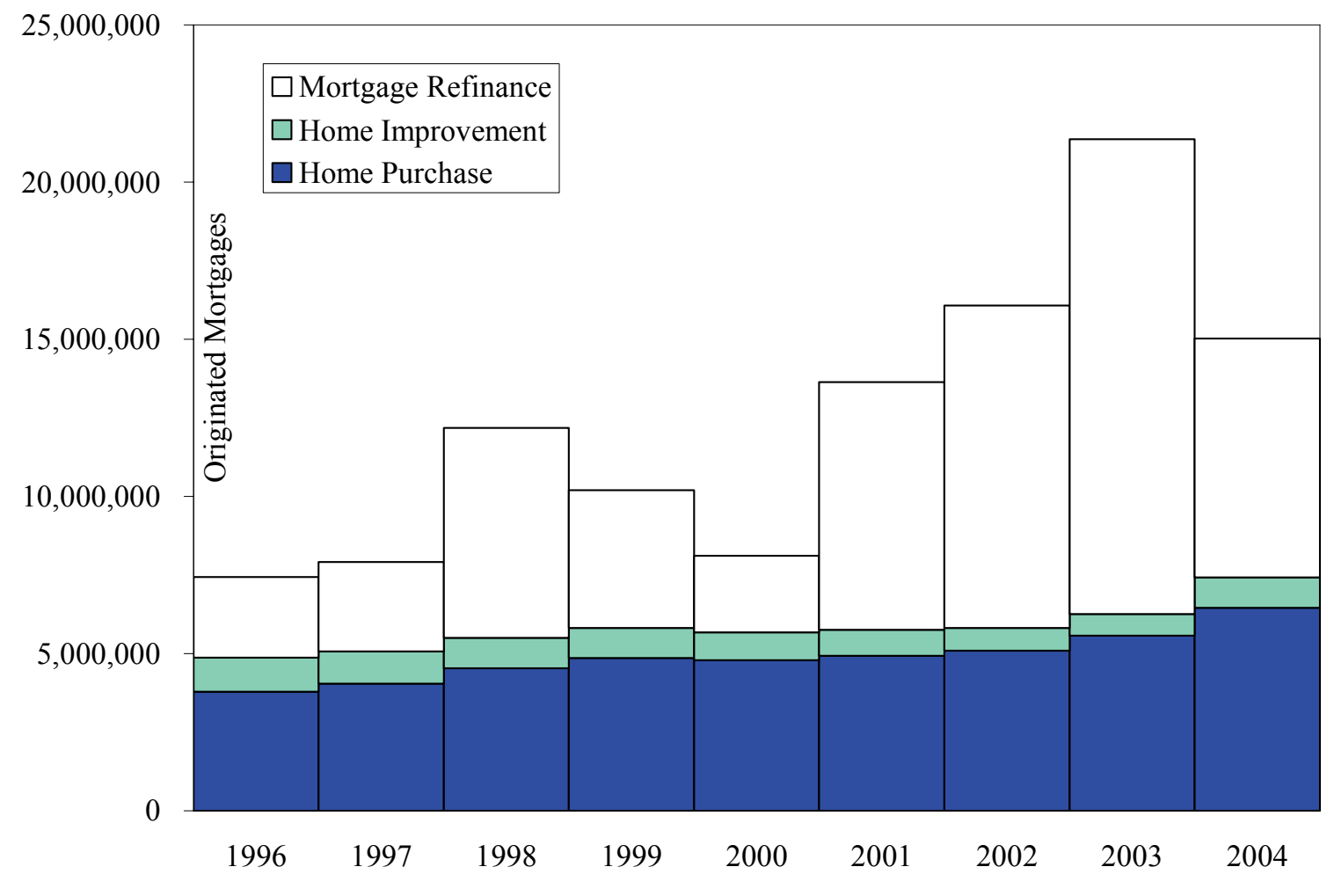

Figure 4. 1996-2004 Trends in Mortgage Originations

Falling interest rates have also contributed to the clear shift toward conventional mortgage products evident in Figure 5. Federal home loan programs have been a means for some disadvantaged segments of the market to acquire home financing. These include Veterans Administration (VA) loan products, Federal Housing Administration (FHA) loan products, and Farm Service Agency (FSA) or Rural Housing Service (RHS) loan products. This shift occurs in falling rate environments because the barriers to home ownership alleviated by the government loan products during higher rate periods are greatly reduced by reducing the cost of borrowing. This means that lenders can use standard (conventional) products to qualify borrowers who previously would have needed the benefits of government loan programs to qualify for a mortgage. 


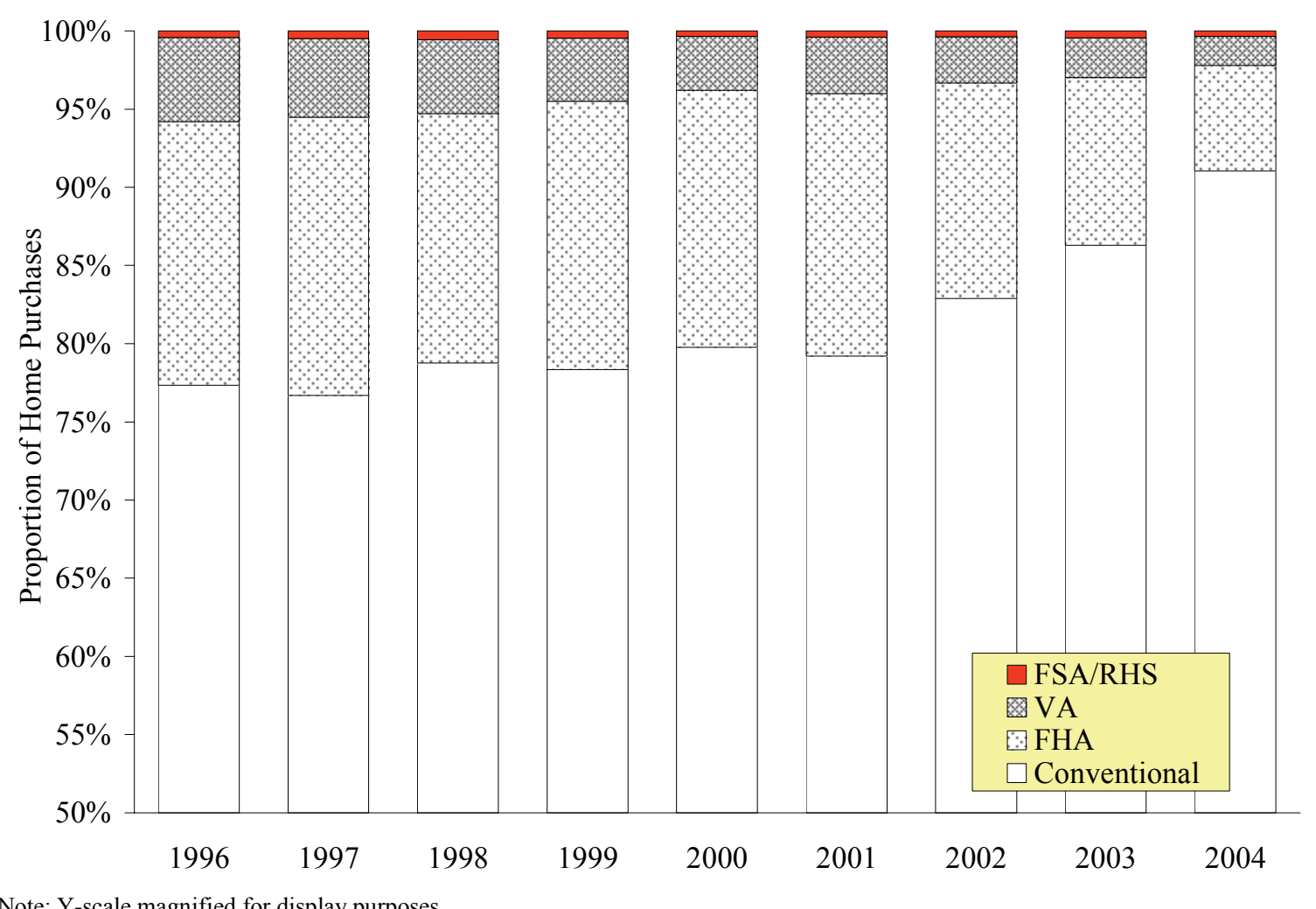

Figure 5. 1996-2004 Trends in Mortgage Products

The HMDA provisions do not require the collection of specific home mortgage products in any finer detail that the classifications shown in Figure 5. Therefore, it is not possible to determine the proportions of subproducts like fixed- versus adjustable-rate mortgages, 15year versus 30-year mortgages, and so on, using the HMDA data.

\section{Mortgage Lending Market Structure}

In a falling-rate environment, demand for home ownership increases. As demand for mortgage financing increases, new mortgage lenders enter the market to handle excess demand. Figure 6 illustrates the segmentation of the lending industry during the study period. The number of HMDA-reporting lenders has fluctuated between 7000 and 8000 over the 1996-2004 period. In general, the top ten lenders, based on number of conventional home mortgages financed, have declined in their share of the market. The second and third tiers of the market have slightly increased their shares over the same period. The top 50 lenders originate roughly $60-65 \%$ of the home purchase mortgages in the country. The remaining thousands of lenders originate the balance. The study period reflects a corresponding period of banking deregulation that has seen a very large number of mergers and acquisitions among the largest banking corporations. We have attempted to account for as many of the large acquisitions as possible for the study period. We consulted various trade publications (Lexis-Nexis 2006, Mortgage Mag 2006), government registries (FTC 2006, FRS 2006), and corporate websites to determine the nature and timing of major merger and acquisition activity in the industry. Using these sources, results in this report are presented by banking corporation. This means that all mortgage activity by all subsidiaries 
or acquired institutions are summarized at the corporation level. For example, during the study period, Washington Mutual Incorporated acquired Great Western Bank, American Savings Bank, Home Savings of America, Long Beach Mortgage, PNC Mortgage Bank, and Dime Bank, among others. This means that results presented by banking corporation will include all the transactions of Washington Mutual operating as the merged institution and all transactions of all predecessor lenders (and their acquisitions) that have subsequently been acquired by Washington Mutual - the current corporation.

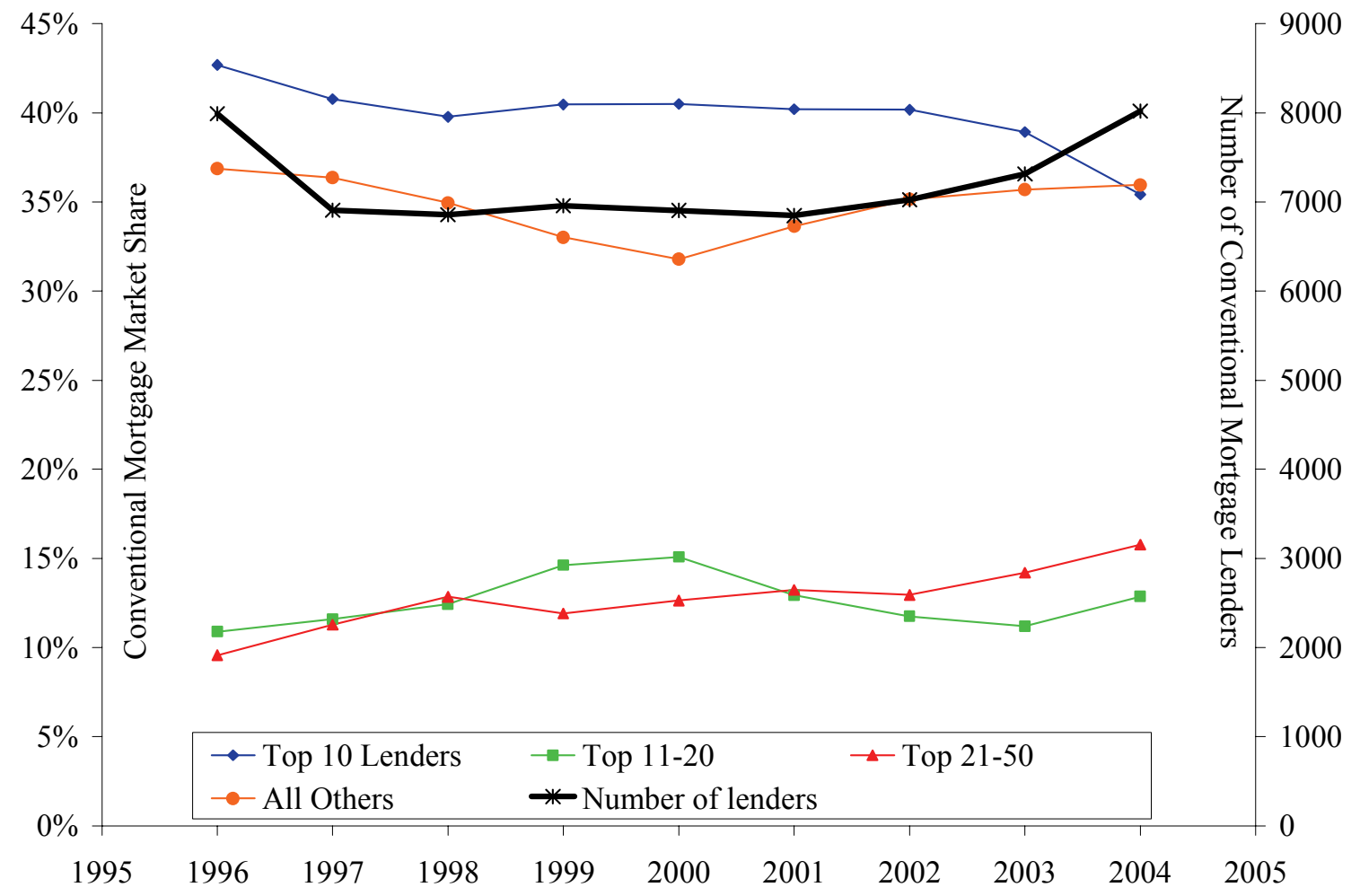

Figure 6. 1996-2004 Market Share Trends in the Conventional Home Purchase Mortgage Market

\section{Mortgage Data Inform Building America}

The Building Technologies Program (BTP) in EERE is home to the Building America program (BA), a Federal program that seeks to accelerate the adoption of advanced technologies in the residential sector by partnering with large builders around the country and getting them to use optimized packages of energy-efficient materials, appliances, and practices in the construction of new homes. Typically these builders develop entire subdivisions and housing communities utilizing the plans developed by the Building America program.

The largest of these communities cover entire census tracts or multiple tracts in some cases. That is, BA communities generally make up a significant portion of the housing units in the census tract(s) in which they are located. We utilized data from the BA program (EEREBTP 2006) to identify the largest BA communities in the country and matched their locations to the appropriate census tract(s). This permits the analysis of HMDA mortgage data specific 
to those tracts, and therefore BA communities generally. Table 15 highlights many of the largest BA communities around the country. This subset of the HMDA data has been analyzed for comparison with other geographic regions presented elsewhere in the report.

Table 15. Home Purchase Mortgage Activity in Building America Census Tracts (Loans)

\begin{tabular}{|c|c|c|c|c|c|c|c|c|c|}
\hline Community & 1996 & 1997 & 1998 & 1999 & 2000 & 2001 & 2002 & 2003 & 2004 \\
\hline Acacia Landing, AZ & 85 & 84 & 104 & 110 & 122 & 265 & 178 & 189 & 194 \\
\hline Adobe Highlands, AZ & 130 & 151 & 241 & 333 & 311 & 464 & 438 & 1,461 & 1,638 \\
\hline Arlington Estates, AZ & 63 & 56 & 67 & 117 & 141 & 153 & 369 & 1,061 & 2,424 \\
\hline Ashton Ranch, AZ & 18 & 64 & 316 & 527 & 884 & 1,338 & 1,578 & 1,919 & 5,128 \\
\hline Cambridge Estates, AZ & 62 & 72 & 102 & 71 & 64 & 157 & 539 & 842 & 1,447 \\
\hline Canyon Trails, AZ & 4 & 2 & 4 & 4 & 8 & 19 & 19 & 338 & 355 \\
\hline Carillon Lakes, IL & 124 & 123 & 177 & 182 & 186 & 185 & 231 & 439 & 504 \\
\hline Civano, AZ & 127 & 127 & 138 & 178 & 202 & 224 & 203 & 762 & 814 \\
\hline El Rancho Grande, NM & 233 & 240 & 250 & 324 & 299 & 317 & 332 & 490 & 668 \\
\hline Estrella Mountain Ranch, AZ & 100 & 109 & 114 & 82 & 93 & 121 & 150 & 508 & 797 \\
\hline Grand Haven, FL & 363 & 422 & 494 & 492 & 563 & 635 & 730 & 938 & 802 \\
\hline Hidden Springs, ID & 124 & 109 & 104 & 98 & 138 & 212 & 191 & 262 & 210 \\
\hline Mainland Square, TX & 102 & 84 & 96 & 117 & 129 & 151 & 151 & 333 & 329 \\
\hline Maple Lawn, WI & 100 & 100 & 106 & 108 & 122 & 156 & 162 & 179 & 161 \\
\hline Meadowview, CO & 126 & 144 & 181 & 179 & 150 & 99 & 115 & 151 & 135 \\
\hline Mentone, FL & 103 & 87 & 118 & 109 & 111 & 114 & 102 & 127 & 189 \\
\hline Misty Ridge, WI & 86 & 97 & 136 & 150 & 111 & 156 & 137 & 120 & 142 \\
\hline On Top of the World, FL & 71 & 70 & 115 & 108 & 148 & 145 & 140 & 180 & 299 \\
\hline Playa Vista, CA & 30 & 22 & 33 & 43 & 31 & 42 & 50 & 397 & 480 \\
\hline Prairie Crossing, IL & 304 & 346 & 354 & 453 & 377 & 446 & 450 & 434 & 605 \\
\hline Solera at Oak Valley Greens, CA & 27 & 34 & 47 & 58 & 62 & 106 & 137 & 206 & 248 \\
\hline Springfiled Golf Resort, AZ & 108 & 125 & 155 & 173 & 237 & 356 & 449 & 271 & 170 \\
\hline Stallion Mountain, NV & 7 & 10 & 7 & 9 & 10 & 12 & 12 & 163 & 173 \\
\hline Stevens Springs, ID & 128 & 172 & 202 & 162 & 187 & 178 & 139 & 248 & 344 \\
\hline Summerset at Frick Park, PA & 52 & 47 & 52 & 79 & 68 & 65 & 95 & 87 & 82 \\
\hline Sun Groves, AZ & 180 & 209 & 258 & 289 & 397 & 596 & 750 & 2,525 & 3,792 \\
\hline Waterford, NV & 37 & 36 & 28 & 36 & 38 & 57 & 83 & 85 & 104 \\
\hline Totals & 2,899 & 3,148 & 4,006 & 4,599 & 5,200 & 6,779 & 7,941 & 18,210 & 25,346 \\
\hline
\end{tabular}

Note: The data pertain to entire census tracts occupied by the BA communities listed, and are reflective of all home buying in these tracts, not just BA home purchases.

Two types of observations appear from examining Table 15. First, because BA communities are new housing developments, they - along with other non-BA developments - are filling previously undeveloped land or land that was not being used for residential development. For example, for Stallion Mountain in Las Vegas, and for Canyon Trails in Phoenix, mortgage activity goes from nearly nothing to significant numbers during the study period. The second observation indicates that in cases where the BA communities are not the only new housing or where a BA community is filling in areas adjacent to existing housing developments, a pronounced "bump" in mortgage activity can be seen. For example, in the case of the Springfield Golf Resort community near Chandler, Arizona, mortgage activity appears to steadily increase as other developments are built in that census tract during the period. Then a pronounced increase in mortgage activity occurs beginning in 2000 and subsiding in 2003. This may be the impact of the construction of the BA community.

We examined the data on lenders to determine which lenders are the most active in the census tracts of the largest BA communities. The results are summarized in Table 16. Not surprisingly, the largest mortgage lenders in the country also dominate home purchase 
lending in the BA communities. These include Wells Fargo, National City, Countrywide, Washington Mutual, JP Morgan-Chase, and Bank of America. Other national and regional lenders also originate a significant number of loans in these communities. Specialty lenders such as Pulte Mortgage and Lennar are the mortgage operations side of their larger home building businesses. Pulte and Lennar are significant Building America home building partners.

Table 16. Top Home Purchase Mortgage Originators in Building America Census Tracts (Loans)

\begin{tabular}{|c|c|c|c|c|c|c|c|c|c|c|}
\hline Banking Corporation & 1996 & 1997 & 1998 & 1999 & 2000 & 2001 & 2002 & 2003 & 2004 & Total \\
\hline Wells Fargo & 233 & 293 & 366 & 409 & 417 & 573 & 639 & 1,635 & 1,862 & 6,429 \\
\hline National City & 85 & 77 & 127 & 220 & 367 & 566 & 603 & 1,263 & 1,194 & 4,501 \\
\hline Countrywide & 114 & 155 & 209 & 206 & 274 & 342 & 360 & 1,007 & 1,422 & 4,089 \\
\hline Washington Mutual & 233 & 248 & 257 & 295 & 301 & 407 & 231 & 573 & 671 & 3,216 \\
\hline JP Morgan Chase & 245 & 251 & 243 & 209 & 206 & 205 & 200 & 379 & 567 & 2,505 \\
\hline Lennar & 36 & 38 & 71 & 127 & 226 & 236 & 314 & 506 & 820 & 2,373 \\
\hline Bank of America & 141 & 147 & 135 & 139 & 167 & 186 & 167 & 417 & 593 & 2,092 \\
\hline Allied Irish Banks & 84 & 66 & 43 & 57 & 41 & 54 & 131 & 554 & 807 & 1,837 \\
\hline KB Home Mortgage & 15 & 30 & 100 & 153 & 230 & 336 & 302 & 408 & 231 & 1,806 \\
\hline First Horizon & 33 & 58 & 223 & 240 & 95 & 64 & 93 & 262 & 540 & 1,609 \\
\hline Pulte Mortgage & 34 & 16 & 23 & 31 & 31 & 83 & 151 & 871 & 347 & 1,586 \\
\hline Home American Mortgage & 24 & 28 & 38 & 39 & 66 & 122 & 212 & 613 & 435 & 1,576 \\
\hline Citibank & 63 & 69 & 44 & 46 & 77 & 99 & 197 & 361 & 587 & 1,543 \\
\hline First Magnus Financial & - & - & - & 18 & 30 & 55 & 64 & 302 & 1,021 & 1,489 \\
\hline Suntrust & 30 & 48 & 78 & 79 & 65 & 66 & 149 & 393 & 510 & 1,418 \\
\hline ABN AMRO & 42 & 45 & 67 & 100 & 126 & 200 & 227 & 320 & 261 & 1,389 \\
\hline Centex & 40 & 46 & 61 & 92 & 125 & 176 & 177 & 230 & 238 & 1,184 \\
\hline Ryland Mortgage & 2 & 6 & 17 & 51 & 29 & 90 & 164 & 244 & 452 & 1,055 \\
\hline Shea Mortgage & - & - & - & - & - & 42 & 136 & 392 & 464 & 1,035 \\
\hline GMAC & 20 & 18 & 24 & 31 & 22 & 64 & 128 & 302 & 339 & 946 \\
\hline Totals & 1,473 & 1,637 & 2,128 & 2,540 & 2,895 & 3,968 & 4,644 & 11,032 & 13,361 & 43,678 \\
\hline
\end{tabular}

Note: The data pertain to entire census tracts occupied by the BA communities listed, and are reflective of all home buying in these tracts, not just BA home purchases.

It also may be useful to view trend information based on the climate regions used by the BA program. Briggs et al. (2002a, 2002b) provided the underlying geographical classification of climate regions adapted by the BA program. These regions are adaptations of the regions adopted by the International Energy Conservation Code (IECC) in 2006 - see Figure 3. Table 17 indicates the annual volume of home purchase mortgages by climate region. Table 18 provides shares of home purchase activity by climate region. 
Table 17. 1996-2004 SF Home Purchase Mortgage Volume by Building America Climate Region

\begin{tabular}{|l|r|r|r|r|r|r|r|r|r|}
\hline Region & \multicolumn{1}{|c|}{1996} & \multicolumn{1}{c|}{1997} & \multicolumn{1}{c}{1998} & \multicolumn{1}{c}{1999} & \multicolumn{1}{c}{2000} & \multicolumn{1}{c|}{2001} & \multicolumn{1}{c|}{2002} & \multicolumn{1}{c|}{2003} & 2004 \\
\hline Cold & $1,231,282$ & $1,271,551$ & $1,458,051$ & $1,553,906$ & $1,529,444$ & $1,555,047$ & $1,599,141$ & $1,715,896$ & $1,923,153$ \\
\hline Hot-Dry & 413,048 & 458,145 & 557,395 & 614,314 & 641,947 & 690,767 & 765,276 & 857,219 & $1,030,237$ \\
\hline Hot-Humid & 438,937 & 465,284 & 558,079 & 599,194 & 653,320 & 688,534 & 666,801 & 801,511 & 952,860 \\
\hline Marine & 197,846 & 244,349 & 282,487 & 296,293 & 280,496 & 266,565 & 298,800 & 328,932 & 380,328 \\
\hline Mixed-Dry & 23,566 & 25,289 & 30,611 & 31,656 & 32,267 & 34,767 & 37,354 & 40,660 & 48,167 \\
\hline Mixed-Humid & $1,177,853$ & $1,371,008$ & $1,474,306$ & $1,563,520$ & $1,533,825$ & $1,564,478$ & $1,575,648$ & $1,723,662$ & $1,985,372$ \\
\hline Subarctic & 633 & 770 & 974 & 957 & 826 & 914 & 901 & 1,098 & 1,588 \\
\hline Very Cold & 22,498 & 26,671 & 31,997 & 31,675 & 31,452 & 32,451 & 34,423 & 38,028 & 43,814 \\
\hline Total & $3,505,663$ & $3,863,067$ & $4,393,900$ & $4,691,515$ & $4,703,577$ & $4,833,523$ & $4,978,344$ & $5,507,006$ & $6,365,519$ \\
\hline
\end{tabular}

Table 18. 1996-2004 SF Home Purchase Shares by Building America Climate Region

\begin{tabular}{|l|r|r|r|r|r|r|r|r|r|}
\hline Region & 1996 & 1997 & 1998 & 1999 & 2000 & 2001 & 2002 & 2003 & 2004 \\
\hline Cold & $35.12 \%$ & $32.92 \%$ & $33.18 \%$ & $33.12 \%$ & $32.52 \%$ & $32.17 \%$ & $32.12 \%$ & $31.16 \%$ & $30.21 \%$ \\
\hline Hot-Dry & $11.78 \%$ & $11.86 \%$ & $12.69 \%$ & $13.09 \%$ & $13.65 \%$ & $14.29 \%$ & $15.37 \%$ & $15.57 \%$ & $16.18 \%$ \\
\hline Hot-Humid & $12.52 \%$ & $12.04 \%$ & $12.70 \%$ & $12.77 \%$ & $13.89 \%$ & $14.24 \%$ & $13.39 \%$ & $14.55 \%$ & $14.97 \%$ \\
\hline Marine & $5.64 \%$ & $6.33 \%$ & $6.43 \%$ & $6.32 \%$ & $5.96 \%$ & $5.51 \%$ & $6.00 \%$ & $5.97 \%$ & $5.97 \%$ \\
\hline Mixed-Dry & $0.67 \%$ & $0.65 \%$ & $0.70 \%$ & $0.67 \%$ & $0.69 \%$ & $0.72 \%$ & $0.75 \%$ & $0.74 \%$ & $0.76 \%$ \\
\hline Mixed-Humi & $33.60 \%$ & $35.49 \%$ & $33.55 \%$ & $33.33 \%$ & $32.61 \%$ & $32.37 \%$ & $31.65 \%$ & $31.30 \%$ & $31.19 \%$ \\
\hline Subarctic & $0.02 \%$ & $0.02 \%$ & $0.02 \%$ & $0.02 \%$ & $0.02 \%$ & $0.02 \%$ & $0.02 \%$ & $0.02 \%$ & $0.02 \%$ \\
\hline Very Cold & $0.64 \%$ & $0.69 \%$ & $0.73 \%$ & $0.68 \%$ & $0.67 \%$ & $0.67 \%$ & $0.69 \%$ & $0.69 \%$ & $0.69 \%$ \\
\hline
\end{tabular}

The data from Table 18 have been charted in Figure 7. The proportion of home purchase mortgage activity occurring in the Cold and Mixed-Humid climate regions appears to be in decline, while the proportions for the Hot-Dry and Hot-Humid climate regions appear to be increasing. This corresponds to the continuing population growth and migration to warm climes of the country - the Sun Belt extending from Texas to Florida along the gulf coast, and the southwestern deserts extending from California to Texas. The mortgage data suggest that this migration continues unabated. The other regions appear to be somewhat stable in terms of proportion to the other regions of the country. 


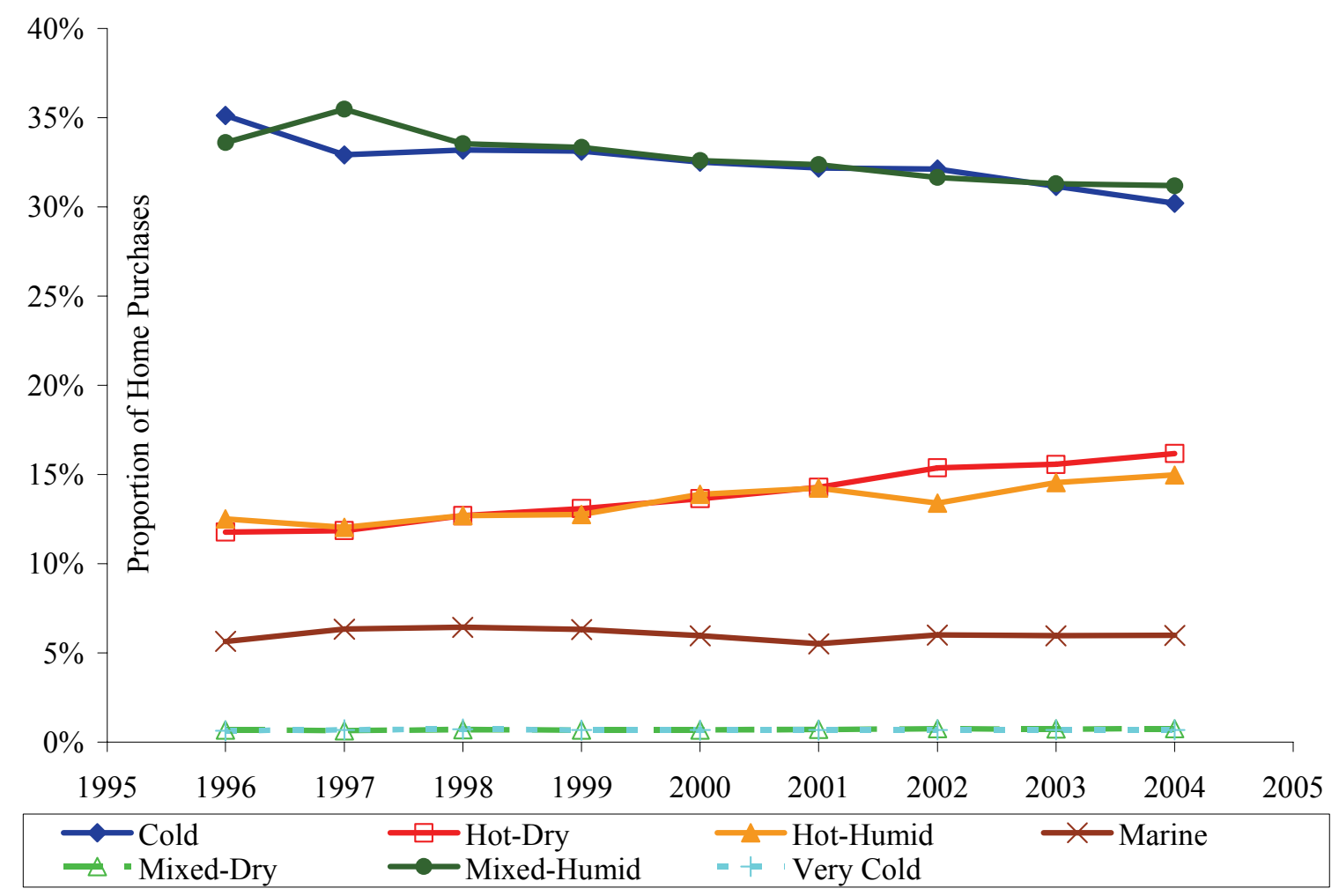

Figure 7. 1996-2004 Proportion of Home Buying by Building America Climate Region

\section{Geography of Lender Market Share}

To gain a further geographic snapshot of the structure of the home purchase financing market, first we segmented the market into quartiles based on the number of single-family home purchase mortgages originated in 2004 from the HMDA data. These quartiles are summarized in Table 19. Each quartile represents roughly 25 percent of the number of originated mortgages in 2004 , by definition.

Table 19. 2004 Market Share Quartile Constituents

\begin{tabular}{|c|l|}
\hline $\begin{array}{c}2004 \\
\text { Quartile }\end{array}$ & \multicolumn{1}{|c|}{ Lending Corporation } \\
\hline \multirow{4}{*}{$1^{\text {st }}$ Tier } & Bank of America \\
\cline { 2 - 2 } & Countrywide \\
\cline { 2 - 2 } & National City \\
\cline { 2 - 2 } & Washington Mutual \\
\cline { 2 - 2 } & Wells Fargo \\
\hline $2^{\text {nd }}$ Tier & ABN AMRO \\
\cline { 2 - 2 } & American Home Mortgage \\
\cline { 2 - 2 } & Argent Mortgage \\
\cline { 2 - 2 } & Centex \\
\cline { 2 - 2 } & Citibank \\
\cline { 2 - 2 } & First Horizon Bank \\
\cline { 2 - 2 } & \\
\hline
\end{tabular}




\begin{tabular}{|c|c|}
\hline $\begin{array}{c}2004 \\
\text { Quartile }\end{array}$ & Lending Corporation \\
\hline \multirow{12}{*}{$\begin{array}{c}2^{\text {nd }} \text { Tier } \\
\text { Continued }\end{array}$} & Flagstar Bank \\
\hline & Fremont Investment \& Loan \\
\hline & GMAC \\
\hline & HSBC \\
\hline & JP Morgan Chase \\
\hline & New Century Mortgage \\
\hline & North Fork Bank \\
\hline & Option One Mortgage \\
\hline & PNC Bank \\
\hline & Suntrust Bank \\
\hline & Wachovia \\
\hline & World Savings \\
\hline $3^{\text {rd }}$ Tier & 111 Lenders \\
\hline $4^{\text {th }}$ Tier & 7923 Lenders \\
\hline
\end{tabular}

From Table 19, the national structure of the home finance market becomes apparent. The top five lenders in the country account for 25 percent of the home purchase loans originated in 2004. The next 18 lenders account for the second 25 percent. The next 111 lenders account for the third 25 percent, and nearly 8,000 lenders account for the final 25 percent. Each of first-tier lenders is a large corporation with national reach and presence in all parts of the country - and may operate under several franchises. In the second tier, a few large regional lenders start to enter the structure, such as Suntrust Bank (The Southeast), North Fork Bank (CT, NJ, NY), and World Savings (California), but the tier is still dominated by national home lenders like Wachovia, GMAC, and Citibank. The third tier is made up largely of significant regional lenders. The fourth tier consists largely of local banks and small independent mortgage companies.

After segmenting the market into national quartiles, we further segmented at the state and county level. For each state and county in the country we determined which tier of the market $\left(1^{\text {st }}, 2^{\text {nd }}, 3^{\text {rd }}\right.$, or $\left.4^{\text {th }}\right)$ was the market leader and how strong that leadership was in terms of the leading tier's share of the county's home purchase loans originated. These results appear on Map A.9 and Map A.10 in the Map Appendix.

Several observations are possible from examination of the national county-level and statelevel maps of market share results. The first-tier lenders do not dominate the country geographically. Their influence is strongest on the west coast, Hawaii, and in the intermountain states. These are significant growth areas as continued migration drives strong demand for housing. These are also areas where home values are high relative to the rest of the country, which leads to relatively higher margins for each loan originated. Perhaps more interestingly, the fourth tier is the market leader for the largest geographic expanse. Much of the Plains, Midwest, and South are dominated by local banks and mortgage lenders with notable exceptions. In large metropolitan centers like Atlanta, Dallas, Houston, Chicago, 
Detroit, Cleveland, Miami, and along the eastern seaboard, second- and third-tier lenders lead the market. Local lenders still lead the majority of rural America in mortgage market share. The second-tier lenders appear to be strongest in the Northeast, Florida, Chicagoland, and Michigan. The third-tier lenders lead the market in Phoenix, Las Vegas, Dallas, Houston, Atlanta, Indianapolis, and Raleigh-Durham.

A similar geographic analysis is reported for home improvement loans in Map A.11, based on the home purchase lender tiers. First-tier lenders dominate much more of the geography in the home improvement lending market. Second-tier lenders appear to be strongest in the Mid-Atlantic (VA, MD, DC, NC, DE, PA). Third-tier lenders appear to be strongest in the Midwest and Deep South (MS, LA). Fourth-tier lenders are still prevalent in rural America, but much less so than in the home purchase market. They also lead in some large metropolitan areas (Boston, Chicago, Denver).

Table 20 illustrates the influence of each tier on the census tracts of Building America developments. Third-tier lenders currently lead the home purchase market in the BA census tracts.

Table 20. Building America Census Tracts Single-Family Home Purchase Lending Market Tiers

\begin{tabular}{|c|r|r|}
\hline $\begin{array}{c}\text { SF Market } \\
\text { Share Tier }\end{array}$ & $\begin{array}{r}\text { Home } \\
\text { Purchases }\end{array}$ & Percent \\
\hline $1^{\text {st }}$ & 5,742 & $22.7 \%$ \\
\hline $2^{\text {nd }}$ & 5,663 & $22.3 \%$ \\
\hline $3^{\text {rd }}$ & 8,526 & $33.6 \%$ \\
\hline $4^{\text {th }}$ & 5,415 & $21.4 \%$ \\
\hline Total & 25,346 & $100.0 \%$ \\
\hline
\end{tabular}

\section{Income Trends Derived from Mortgage Data}

Perhaps the most valuable data provided in the HMDA reporting are the borrower income data. The income values are those used to qualify for a home purchase mortgage. For regulatory examination purposes, the agencies that regulate the HMDA-reporting institutions compare the borrower income to the HUD Median Family Income for the specific county or metropolitan area of the property being financed. This ratio is used to classify borrowers as low-, moderate-, middle-, or upper-income. The specific income classifications appear below:

- Low $=$ Less than $50 \%$ of area median family income

- Moderate $=50 \% \leq$ area median family income $<80 \%$

- Middle $=80 \% \leq$ area median family income $<120 \%$

- Upper $=\geq 120 \%$ of area median family income

For summarization purposes and consistency with banking industry reporting, we utilize this classification to report income information in this report. Because we are always comparing to the area median income, borrower income is always normalized to that year's median 
income. The principal benefit of using the ratio is that it nulls the effect of inflation on the dollar values in the data. The estimation of HUD Median Family Income can be found in HUD (2006). "Family" refers to the Census definition of a family, which is a householder with one or more other persons living in the same household who are related to the householder by birth, marriage, or adoption. The definition of family excludes one-person households and multi-person households of unrelated individuals (HUD 2006). Table 21 provides the summary of home purchase lending by income class. These volumes of loans seed the proportions presented in Table 22. The data bear out the obvious conclusion that ability to purchase a home corresponds to income, as nearly half of all home purchase mortgages are obtained by upper-income borrowers alone.

Table 21. 1996-2004 Home Purchase Loans by Borrower Income Class

\begin{tabular}{|l|r|r|r|r|r|r|r|r|r|}
\hline Income Level & 1996 & 1997 & 1998 & 1999 & 2000 & 2001 & 2002 & 2003 & 2004 \\
\hline Low & 236,026 & 273,360 & 318,172 & 381,930 & 363,606 & 355,365 & 371,556 & 404,938 & 396,186 \\
\hline Moderate & 719,406 & 800,779 & 914,241 & $1,017,328$ & 977,685 & 990,933 & $1,030,238$ & $1,109,510$ & $1,236,306$ \\
\hline Middle & 999,563 & $1,072,586$ & $1,201,727$ & $1,269,265$ & $1,233,435$ & $1,263,071$ & $1,296,866$ & $1,418,876$ & $1,633,533$ \\
\hline Upper & $1,483,948$ & $1,630,412$ & $1,851,831$ & $1,922,005$ & $1,974,024$ & $2,008,649$ & $2,094,516$ & $2,325,970$ & $2,828,216$ \\
\hline Total & $3,438,943$ & $3,777,137$ & $4,285,971$ & $4,590,528$ & $4,548,750$ & $4,618,018$ & $4,793,176$ & $5,259,294$ & $6,094,241$ \\
\hline
\end{tabular}

Table 22. Income Characteristics of Home Purchase Mortgagees

\begin{tabular}{|c|c|c|c|c|c|c|c|c|c|}
\hline Income Level & 1996 & 1997 & 1998 & 1999 & 2000 & 2001 & 2002 & 2003 & 2004 \\
\hline Low & $6.9 \%$ & $7.2 \%$ & $7.4 \%$ & $8.3 \%$ & $8.0 \%$ & $7.7 \%$ & $7.8 \%$ & $7.7 \%$ & $6.5 \%$ \\
\hline Moderate & $20.9 \%$ & $21.2 \%$ & $21.3 \%$ & $22.2 \%$ & $21.5 \%$ & $21.5 \%$ & $21.5 \%$ & $21.1 \%$ & $20.3 \%$ \\
\hline Middle & $29.1 \%$ & $28.4 \%$ & $28.0 \%$ & $27.6 \%$ & $27.1 \%$ & $27.4 \%$ & $27.1 \%$ & $27.0 \%$ & $26.8 \%$ \\
\hline Upper & $43.2 \%$ & $43.2 \%$ & $43.2 \%$ & $41.9 \%$ & $43.4 \%$ & $43.5 \%$ & $43.7 \%$ & $44.2 \%$ & $46.4 \%$ \\
\hline Qualified Home Purchases & $3,438,943$ & $3,777,137$ & $4,285,971$ & $4,590,528$ & $4,548,750$ & $4,618,018$ & $4,793,176$ & $5,259,294$ & $6,094,241$ \\
\hline Mortgages with Missing Info & 350,276 & 262,422 & 242,554 & 259,244 & 234,433 & 314,822 & 302,691 & 315,458 & 358,619 \\
\hline All Home Purchase Mortgages & $3,789,219$ & $4,039,559$ & $4,528,525$ & $4,849,772$ & $4,783,183$ & $4,932,840$ & $5,095,867$ & $5,574,752$ & $6,452,860$ \\
\hline Valid Proportion of Mortgages & $90.8 \%$ & $93.5 \%$ & $94.6 \%$ & $94.7 \%$ & $95.1 \%$ & $93.6 \%$ & $94.1 \%$ & $94.3 \%$ & $94.4 \%$ \\
\hline Avg. Proportion of Median Income & 1.432 & 1.472 & 1.447 & 1.425 & 1.471 & 1.461 & 1.670 & 1.489 & 1.552 \\
\hline Percent $>=3 x$ Median Income & $5.7 \%$ & $6.0 \%$ & $6.1 \%$ & $6.0 \%$ & $6.6 \%$ & $6.4 \%$ & $6.8 \%$ & $6.9 \%$ & $7.7 \%$ \\
\hline
\end{tabular}

Note: Missing information in individual mortgage origination data needed for income statistics can include either missing income values or missing or incorrect geo-coding of the county by the submitting institution.

A few observations are possible using Table 22. First, it is important to note that about 5 percent of the mortgage records from the HMDA data are not valid for income analysis as stated in the table note because of either non-reporting of income or incomplete recording of geography by the submitting institution. However, the loss of 5 percent of the home purchase mortgage population of several million records annually is not deemed significant for analysis purposes. Second, the weighted national average ratio of borrower income to median income falls generally in the range of 1.4 to 1.5 . In 2004, the U.S. median family income was $\$ 57,500$. Further, the proportion of home buyers having greater than three times the area median income has been steadily rising over the study period. This could signal that the national income distribution is steadily widening. As a consequence, higher income borrowers can demand more expensive housing, indicated by the trends in Figure 8. Home values - here represented by home purchase mortgage amounts - as a proportion of area median income have been outpacing income growth over the length of the study period, and the rate of outpacing is increasing steadily, as well. These conditions and the associated rate environment have lead to the popular notion of the "housing bubble" in real estate parlance. Because home values continue to outpace family income in real terms and because more families appear to be entering the high end of the income distribution tail, demand for real estate continues to be strong. 


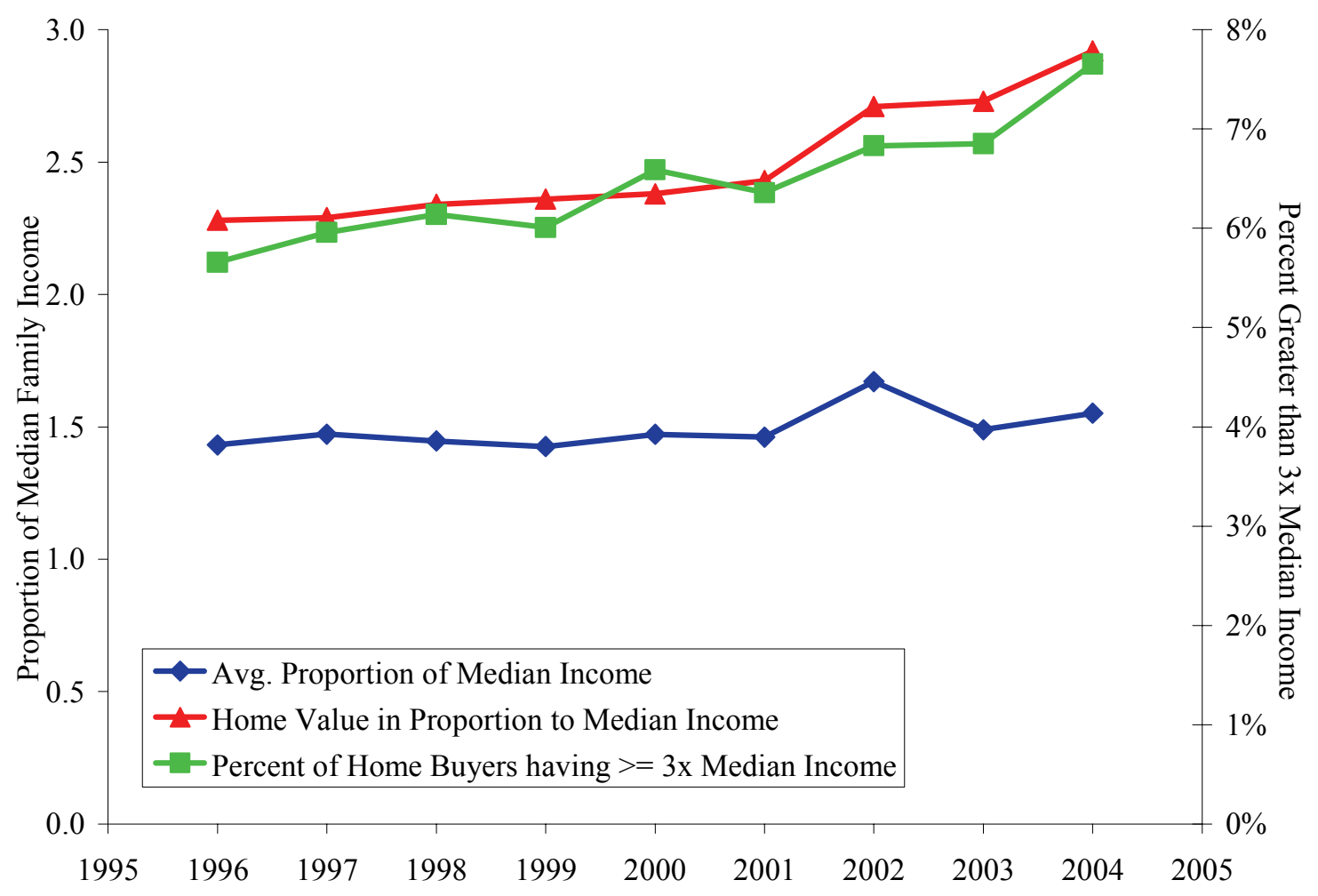

Figure 8. Trends in Family Income Relative to Home Values

Home ownership - here depicted by home purchasing activity - has been increasing steadily in terms of raw numbers of home owners. Figure 9 illustrates the income distribution of home buyers during the study period expressed in terms of the proportion of the area median family income. Because of the extremely long tail of the income distribution, we have truncated it for display purposes. In volume terms, the proportion of home buyers having more than three times the median family income is very large and getting larger. Figure 9 also illustrates the tremendous growth in the numbers of home buyers during the study period. 


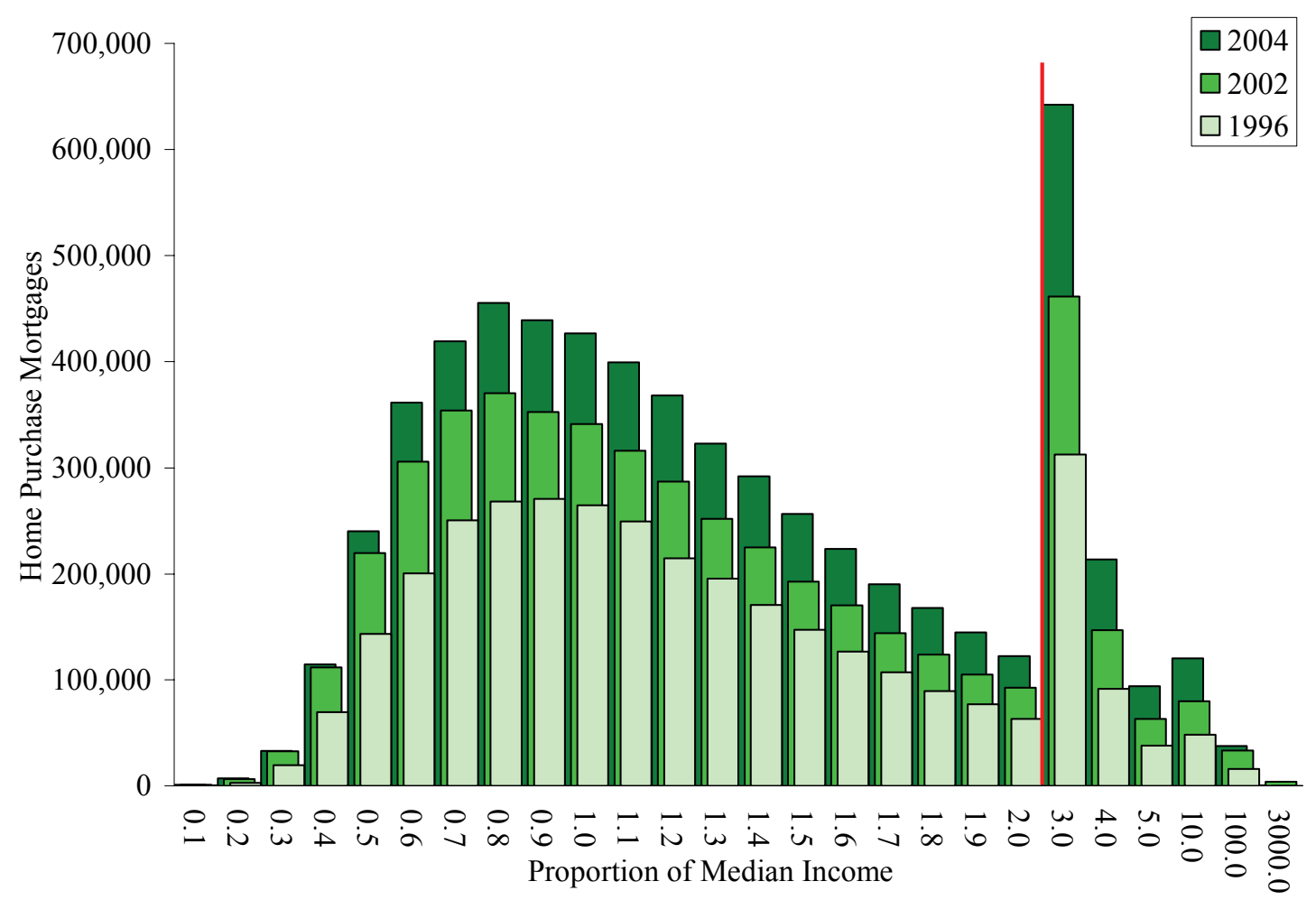

Note: The X-scale has been truncated for display purposes

Figure 9. Income Distribution of Home Buyers in 1996, 2002, and 2004

Drilling into the HMDA data specific to the BA communities, we have determined the family income segment of the top mortgage lenders serving the census tracts of the largest BA communities. Table 23 values indicate that on average, the family income of home buyers in the BA communities has been outpacing the income growth in general. This provides some indication of housing affordability. The steady outpacing of income growth suggests that home values for these areas have grown such that higher and higher incomes will be required over time to afford to live in these communities. That would be consistent with adding new single-family housing to any area previously undeveloped or minimally developed.

Extending the affordability discussion a little further, we also looked at regional differences in home value growth. Using the surrogate measure of the home purchase mortgage amount financed, we plotted home value against area median income in Figure 10 by BA climate region and include a U.S. series and a BA series for comparison. A few observations are possible. First, for the study period the data indicate the relative demand for single-family housing by the starting position of each region on the chart. For example, average home values in the Desert Southwest and along the West Coast were hovering around an average value of three times area median income, but have since risen sharply relative to incomes making housing much less affordable generally in those areas. Affordability is further exacerbated in these regions because median family incomes in California and the western portions of Oregon and Washington are much higher than the national average. Of interest, although home values are outpacing income growth in nearly all regions, the BA communities appear to be reflective of the national average growth in home value relative to income growth. 
Table 23. Annual Average Borrower Income as a Proportion of HUD Median Family Income in Building America Census Tracts by Lender

\begin{tabular}{|l|c|c|c|c|c|c|c|c|c|c|}
\hline Banking Corporation & 1996 & 1997 & 1998 & 1999 & 2000 & 2001 & 2002 & 2003 & 2004 & Average \\
\hline Suntrust & 1.236 & 1.655 & 1.852 & 1.581 & 1.689 & 1.615 & 1.404 & 1.390 & 2.155 & 1.697 \\
\hline Washington Mutual & 1.102 & 1.367 & 1.301 & 1.547 & 1.415 & 1.383 & 1.735 & 1.653 & 1.914 & 1.649 \\
\hline JP Morgan Chase & 1.071 & 1.515 & 1.427 & 1.658 & 1.590 & 1.602 & 1.577 & 1.346 & 1.890 & 1.612 \\
\hline Countrywide & 1.111 & 1.261 & 1.196 & 1.308 & 1.254 & 1.318 & 1.403 & 1.613 & 1.699 & 1.420 \\
\hline Lennar & 1.158 & 1.305 & 1.167 & 1.316 & 1.161 & 1.241 & 1.274 & 1.821 & 1.669 & 1.419 \\
\hline Shea Mortgage & - & - & - & - & - & 1.268 & 1.211 & 1.397 & 1.614 & 1.141 \\
\hline Bank of America & 0.689 & 1.286 & 1.366 & 1.536 & 1.517 & 1.521 & 1.565 & 1.462 & 1.608 & 1.456 \\
\hline Wells Fargo & 1.219 & 1.288 & 1.464 & 1.377 & 1.444 & 1.438 & 1.411 & 1.431 & 1.597 & 1.487 \\
\hline Citibank & 0.920 & 1.258 & 1.387 & 1.432 & 1.620 & 1.560 & 1.577 & 1.579 & 1.583 & 1.547 \\
\hline Centex & 1.067 & 1.332 & 1.241 & 1.257 & 1.147 & 1.300 & 1.121 & 1.086 & 1.570 & 1.318 \\
\hline First Horizon & 1.131 & 1.271 & 1.182 & 1.251 & 1.305 & 1.375 & 1.167 & 1.561 & 1.558 & 1.485 \\
\hline Allied Irish Banks & 1.105 & 1.236 & 1.136 & 1.108 & 1.275 & 1.343 & 1.263 & 1.233 & 1.536 & 1.324 \\
\hline First Magnus & - & - & - & 1.189 & 1.219 & 1.175 & 1.284 & 1.554 & 1.512 & 1.274 \\
\hline National City & 1.168 & 1.256 & 1.283 & 1.241 & 1.226 & 1.377 & 1.332 & 1.355 & 1.442 & 1.411 \\
\hline GMAC & 1.229 & 1.630 & 1.350 & 1.412 & 1.488 & 1.413 & 1.305 & 1.220 & 1.438 & 1.204 \\
\hline Ryland Mortgage & 1.205 & 1.190 & 1.366 & 1.458 & 1.365 & 1.400 & 1.336 & 1.411 & 1.412 & 1.381 \\
\hline Home American Mortgage & 1.003 & 1.030 & 1.220 & 1.406 & 1.215 & 1.197 & 1.157 & 1.325 & 1.361 & 1.255 \\
\hline ABN AMRO & 1.245 & 1.326 & 1.401 & 1.494 & 1.314 & 1.397 & 1.429 & 1.524 & 1.349 & 1.413 \\
\hline Pulte Mortgage & 1.294 & 0.960 & 1.199 & 1.192 & 1.211 & 1.236 & 1.447 & 1.192 & 1.289 & 1.271 \\
\hline KB Home Mortgage & 1.129 & 0.907 & 0.959 & 1.044 & 1.079 & 1.170 & 1.029 & 1.274 & 1.240 & 1.192 \\
\hline Weighted Average & 1.026 & 1.289 & 1.309 & 1.360 & 1.315 & 1.417 & 1.409 & 1.442 & 1.682 & 1.465 \\
\hline
\end{tabular}

Note: The data pertain to entire census tracts occupied by BA communities, and are reflective of all home buying in these tracts, not just BA home purchases.

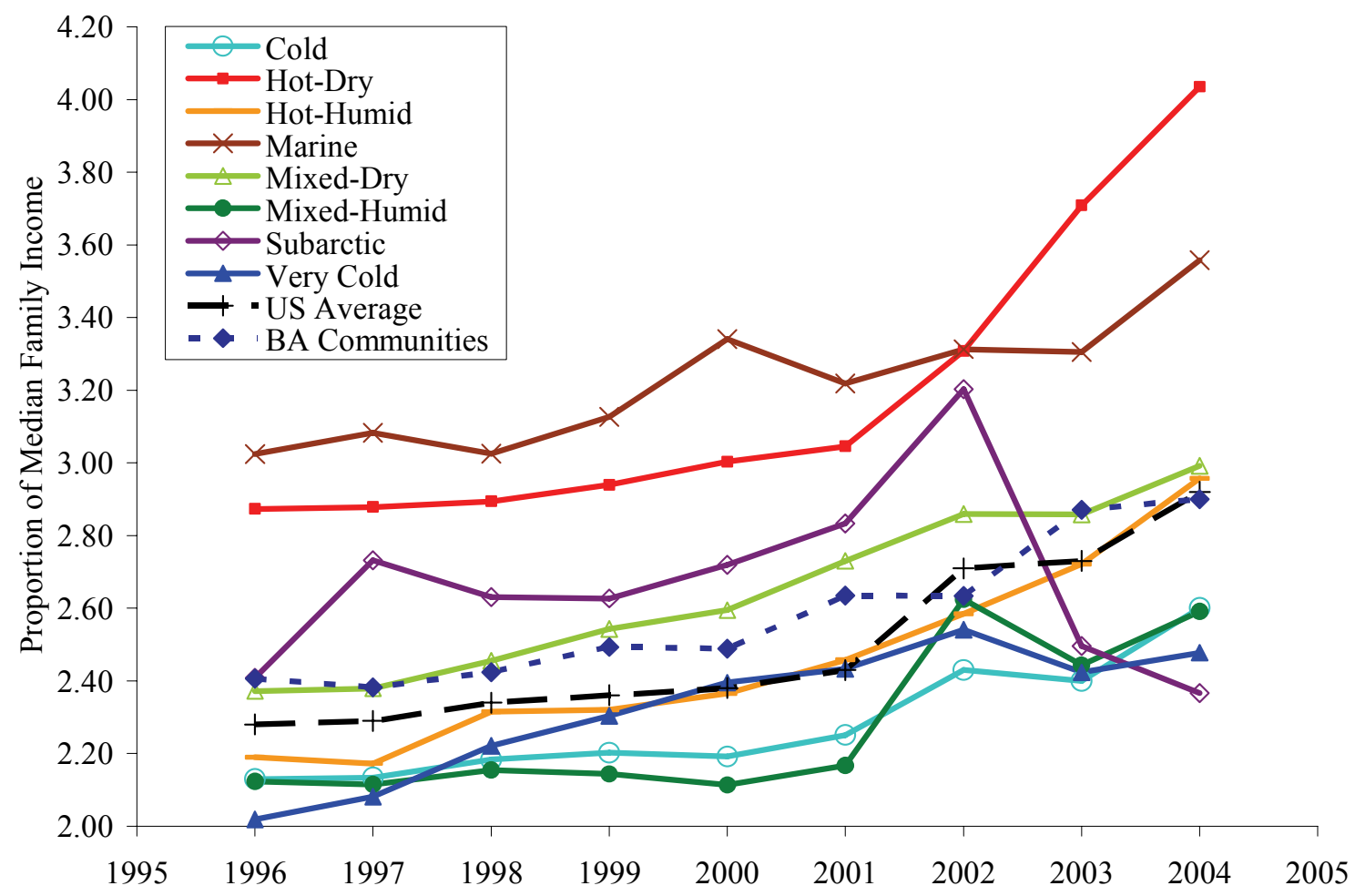

Note: The Y-scale has been magnified for display purposes.

Figure 10. 1996-2004 Home Values as a Proportion of Median Family Income 


\section{Demographic Trends in Home Ownership}

The HMDA data provide a clear view of the changing racial demographics of the United States. In 1996, nearly 80 percent of all home purchases were made by white buyers. By 2004 that number diminished to just over 65 percent. There are several factors contributing to this shift. Both Latino (Latino used in this report and refers to all races having Hispanic ethnicity) and Asian immigrants and their U.S.-born descendants are increasingly able to access credit and acquire a home mortgage. This is a well-documented signal that incomes for these minorities are growing with time and assimilation into American culture. The shift in racial proportions in the home ownership market is in part due to artifacts of technology change in the home loan industry. The number of loans originated on-line or over the phone has been acknowledged to cause a significant increase in the non-reporting of borrower race by the mortgage lender. The increasing share of "not provided" entries in the HMDA data corresponds to the emergence of these new technologies for acquiring home mortgages. Figure 11 below indicates that these responses peaked nationally in 2001, and the HMDA guidance to reporting institutions has suggested remedies since that time to restore more accurate reporting of borrower race and ethnicity - as evidenced by the period since 2001 .

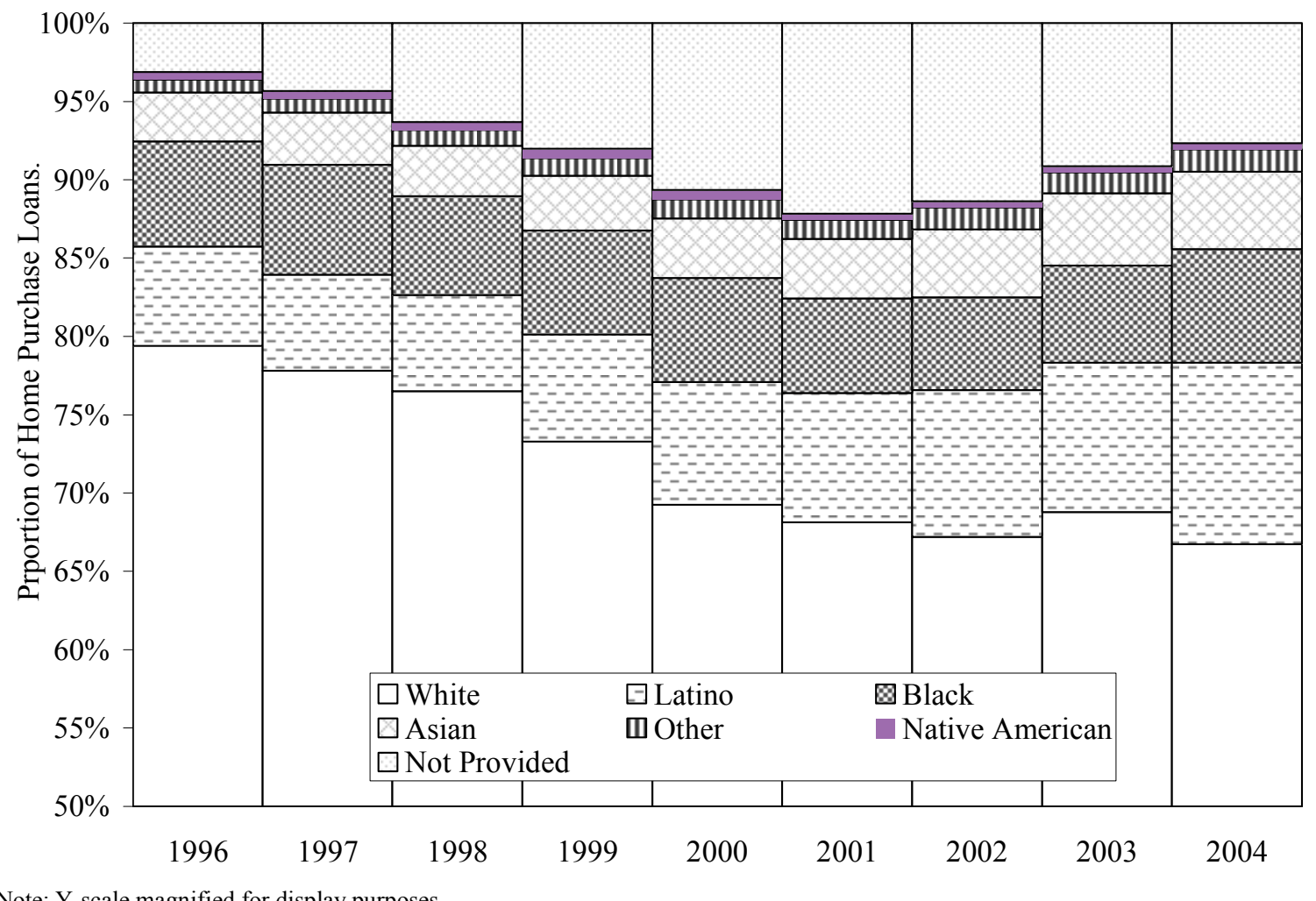

Note: Y-scale magnified for display purposes

Figure 11. Proportion of Home Purchase Mortgages Originated by Race of Borrower

Figure 12 presents the racial demographics for census tracts impacted by Building America developments. The sharp decline in the share of white home buyers is similar to the national trend, but the increase in shares for Latino and Asian home buyers is more pronounced. This is expected based on the heavy weighting of BA community locations to the Desert 
Southwest. Of interest, the trend of unreported race/ethnicity affects a solid 10 percent or more of the home buyer population, and has not receded with the national trend.

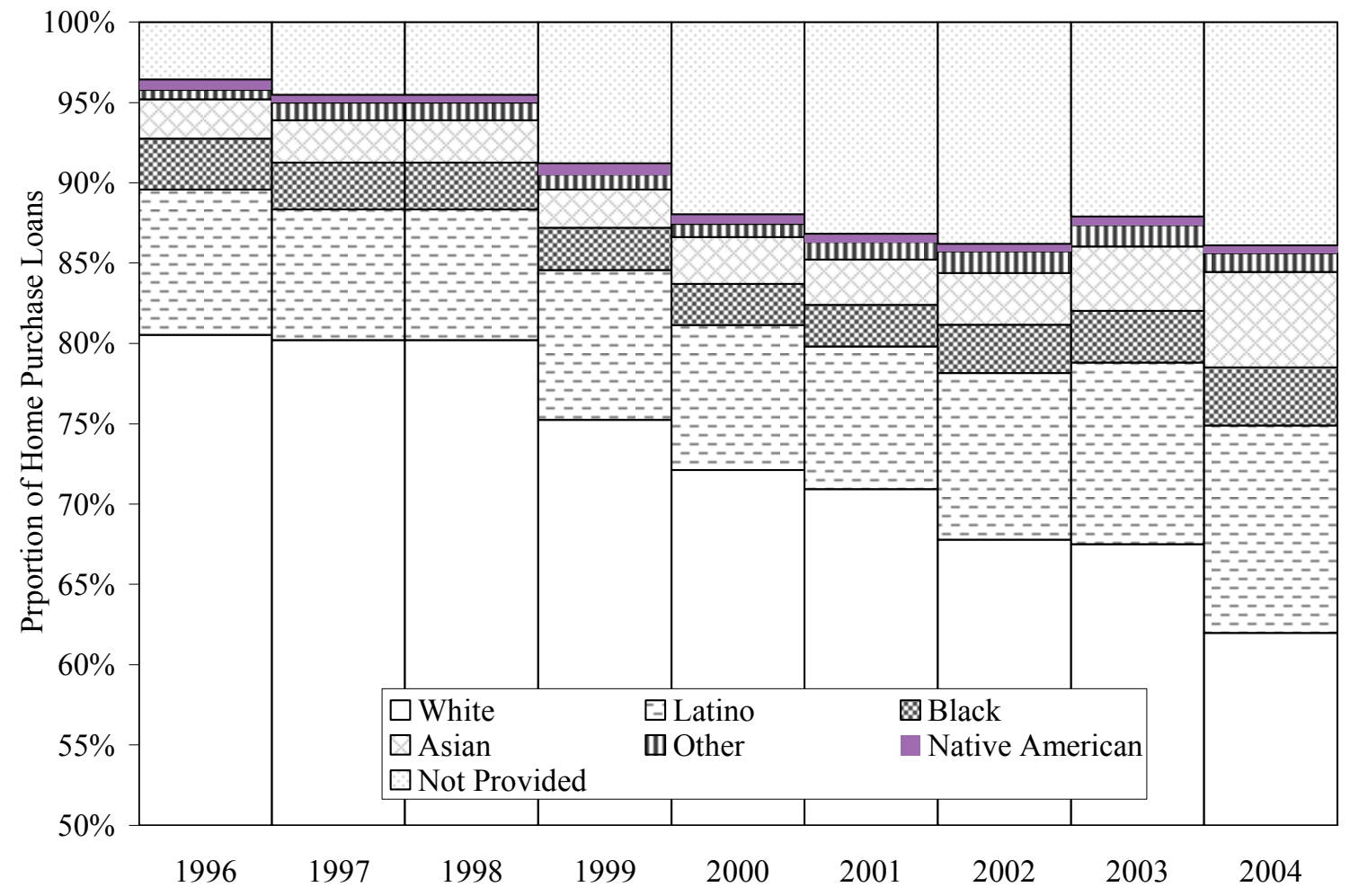

Note: Y-scale magnified for display purposes

Figure 12. Proportion of Home Purchase Mortgages Originated by Race of Borrower in BA Census Tracts.

Racial segmentation of the home buying market also varies widely by climate region as shown in Table 24. Recalling that the fastest growing regions include the Desert Southwest and the Sun Belt, those regions also feature the most racial diversity of the climate regions. These continuing trends pose challenges for the mortgage industry. Financial regulators are concerned with the practice of "subprime" lending. We will treat this topic only briefly to provide some background information.

Table 24. 2004 Home Purchase Proportions by Race and Building America Climate Region

\begin{tabular}{|l|r|r|r|r|r|r|r|r|r|}
\hline Race/Ethnicity & Cold & Hot-Dry & $\begin{array}{c}\text { Hot- } \\
\text { Humid }\end{array}$ & Marine & $\begin{array}{c}\text { Mixed- } \\
\text { Dry }\end{array}$ & $\begin{array}{r}\text { Mixed- } \\
\text { Humid }\end{array}$ & Subarctic & $\begin{array}{r}\text { Very } \\
\text { Cold }\end{array}$ & US \\
\hline Asian & $3.0 \%$ & $8.9 \%$ & $3.6 \%$ & $11.7 \%$ & $2.3 \%$ & $4.2 \%$ & $1.9 \%$ & $1.8 \%$ & $4.9 \%$ \\
\hline Black & $5.1 \%$ & $3.8 \%$ & $7.7 \%$ & $2.2 \%$ & $1.2 \%$ & $12.0 \%$ & $2.1 \%$ & $1.0 \%$ & $7.2 \%$ \\
\hline Latino & $6.5 \%$ & $24.6 \%$ & $18.1 \%$ & $12.0 \%$ & $17.2 \%$ & $6.3 \%$ & $2.9 \%$ & $2.2 \%$ & $11.5 \%$ \\
\hline Native American & $0.3 \%$ & $0.6 \%$ & $0.3 \%$ & $0.5 \%$ & $0.8 \%$ & $0.4 \%$ & $6.3 \%$ & $1.4 \%$ & $0.4 \%$ \\
\hline White & $77.4 \%$ & $49.4 \%$ & $60.4 \%$ & $63.1 \%$ & $69.1 \%$ & $67.0 \%$ & $79.8 \%$ & $88.0 \%$ & $66.2 \%$ \\
\hline Other & $7.0 \%$ & $12.2 \%$ & $8.9 \%$ & $9.9 \%$ & $8.9 \%$ & $9.1 \%$ & $6.5 \%$ & $4.8 \%$ & $8.9 \%$ \\
\hline Not Provided & $0.7 \%$ & $0.6 \%$ & $0.9 \%$ & $0.6 \%$ & $0.5 \%$ & $1.1 \%$ & $0.4 \%$ & $0.8 \%$ & $0.8 \%$ \\
\hline
\end{tabular}




\section{Subprime Lending}

Subprime lending is the practice of charging a cost premium for mortgage customers that pose higher default risks in the eye of the lender. For example, a borrower may demonstrate a very high legitimate income, but may have no credit history or may have a poor credit history. Rather than qualify this borrower for traditional conventional loan products, the lender may hedge its risk of default by charging an interest rate substantially higher than market rates. Beginning with the 2004 HMDA data submission, lenders were required to provide information characteristic of the practice of subprime lending. This includes the spread between the prime rate and the rate charged to the borrower, if that spread was three or more percentage points higher - a de facto definition of a subprime loan (FFIEC 2005a).

The accepted practice of subprime lending is often tied to the discredited practice of "predatory" lending - essentially taking advantage of uninformed borrowers by selling them a subprime mortgage when they might qualify for conventional mortgage products. While detailed analysis of these issues falls outside the scope of this study, Federal programs seeking to partner with mortgage lenders must be aware of the mortgage lending performance of those lenders. Knowing a lender's performance, and particularly its subprime lending performance, would avoid possible future embarrassment if such performance was found to be lower than peers in the industry, or otherwise questionable.

The sensitivity associated with subprime lending can be illustrated using Table 25. Using the HMDA definition of subprime lending, Table 25 illustrates that subprime home purchase mortgages do not follow the same racial proportions as all home purchase mortgages taken together as shown in Table 24. The fact that these mortgages disproportionately serve racial minorities is the cause of concerns by community advocacy groups in many parts of the country and nationally. The reader is directed to research these issues regionally. Further consideration of these impacts, and their potential causes falls beyond the scope of this report.

Table 25. 2004 Subprime Home Purchase Loans by Race of Borrower.

\begin{tabular}{|l|r|r|r|r|}
\hline Race / Ethnicity & $\begin{array}{c}\text { Subprime } \\
\text { Home } \\
\text { Purchases }\end{array}$ & $\begin{array}{c}\text { Subprime } \\
\text { Share }\end{array}$ & $\begin{array}{l}\text { All Home } \\
\text { Purchase } \\
\text { Loans } \\
\text { Share }\end{array}$ & $\begin{array}{c}\text { Percent } \\
\text { Difference } \\
\text { in Shares }\end{array}$ \\
\hline Asian & 29,052 & $3.1 \%$ & $4.9 \%$ & $-35.8 \%$ \\
\hline Black & 145,707 & $15.8 \%$ & $7.2 \%$ & $119.1 \%$ \\
\hline Latino & 182,726 & $19.8 \%$ & $11.5 \%$ & $72.0 \%$ \\
\hline Native American & 6,242 & $0.7 \%$ & $0.4 \%$ & $69.0 \%$ \\
\hline Other or Not Provided & 88,420 & $9.6 \%$ & $9.0 \%$ & $6.7 \%$ \\
\hline White & 471,447 & $51.0 \%$ & $66.2 \%$ & $-22.9 \%$ \\
\hline Total & 923,594 & & & \\
\hline
\end{tabular}

We performed a geographic market share analysis of 2004 subprime home purchase loans, similar to that reported for prime home purchase loans and home improvement loans. The 
results are presented in Map A.12 of the Map Appendix. Using the national home purchase mortgage market share tiers, the results indicate that the first-tier lenders have relatively little presence in the subprime mortgage market or they do not show significant market share geographically. The market is dominated by second- and third-tier lenders. The second-tier lenders are strongest in the Northwest, California's Central Valley, and the Northeast, while the third-tier lenders dominate most of rest of the country's metropolitan areas. Fourth-tier lenders in the subprime market generally are confined to the most rural counties of rural America.

\section{The Emergence of Exurbs}

Another goal of this study was to determine whether the HMDA mortgage data could be used to determine patterns of exurban development on a national scale. Exurban development is a variant of urban sprawl. While urban sprawl is commonly acknowledged to refer to the process of suburban expansion along the fringes of metropolitan areas, exurban development refers to the appearance of relatively disconnected islands of new urban development, somewhat beyond the fringes of suburbia. These islands of residential and some associated services are popularly termed "exurbs", short for "extra-urban".

For this study, we attempted to identify exurbs ex ante using the home purchase data at the census tract level. We have developed a crude classification mechanism based on home buying activity. The ranges specified below were selected arbitrarily to minimize the number of potential census tracts that might satisfy these definitions - providing potentially clear examples of each classification when viewed on a map.

- Significant Emergent: Home purchases go from a minimum of less than 10 to 100 or more within the study period. We suggest that this could indicate the emergence of large new residential areas that could be detached from neighboring developments. (53 census tracts)

- Emergent: Home purchases go from a minimum of 5 or less to between 50 and 100 within the study period. These areas may be similar to the significant emergent tracts, but may be in the middle of a build-out. They may also be simply new small residential areas somewhat isolated from other development. (72 census tracts)

- Significant Infill: Home purchases go from a minimum range of 10 to 100 , to 1000 or more within the study period. We suggest that these tracts have had some small, steady, level of existing home sales that quickly mushroomed due to significant development in the surrounding tracts. (20 census tracts)

- Suburban Expansion: Remaining tracts with a change in home purchases greater than 100 during the study period. (461 census tracts)

These selected census tracts were mapped and appear in Maps A.13-A.21 in the Map Appendix. To examine the effectiveness of the classification, we mapped several metropolitan regions including Northern Virginia, Dallas, Houston, Las Vegas, Denver, Atlanta, the San Francisco Bay Area, and Los Angeles. The exurban characteristics of each are covered in Table 26. 
Table 26. Exurban Characterization of Significantly Growing Urban Areas

\begin{tabular}{|c|c|}
\hline $\begin{array}{c}\text { Metropolitan } \\
\text { Region }\end{array}$ & Exurban Characterization \\
\hline $\begin{array}{l}\text { Atlanta } \\
\text { (Map A.13) }\end{array}$ & $\begin{array}{l}\text { The home purchase data reveal significant suburban expansion at } \\
\text { the far outskirts of the Atlanta metroplex (Polk, Paulding, Butts, } \\
\text { Jasper Cherokee, Borrow, and Jackson Counties). There also } \\
\text { appears to be an inner ring of census tracts identified as suburban } \\
\text { expansion or emergent tracts. Inspection of recent satellite photos } \\
\text { (Microsoft 2006) of the area suggests that these tracts are urban } \\
\text { infill. In several cases, it appears that brownfield (former industrial } \\
\text { site) conversion to residential is underway. Redevelopment } \\
\text { activities around the Turner Field complex may also be contributing } \\
\text { to the increased residential. }\end{array}$ \\
\hline $\begin{array}{l}\text { Dallas } \\
\text { (Map A.14) }\end{array}$ & $\begin{array}{l}\text { The Dallas-Fort Worth metroplex shows classic suburban } \\
\text { expansion, based on the home purchase data. Many of the census } \\
\text { tracts ringing the outer edge of the existing developed region show } \\
\text { increasing levels of home buying. The data do not indicate any } \\
\text { exurbs, although there have been some significant infill or } \\
\text { brownfield conversion activities near downtown Dallas. }\end{array}$ \\
\hline $\begin{array}{l}\text { Denver } \\
\text { (Map A.15) }\end{array}$ & $\begin{array}{l}\text { Eastward expansion of the Denver metro area appears to be } \\
\text { continuing. Development around the Denver International Airport } \\
\text { began with the airport's opening in 1995. This is a truly exurban } \\
\text { community by design (DIA Partnership, 2005). Redevelopment of } \\
\text { the former Stapleton Airport represents residential infill of a } \\
\text { converted land use. }\end{array}$ \\
\hline $\begin{array}{l}\text { Houston } \\
\text { (Map A.16) }\end{array}$ & $\begin{array}{l}\text { Houston displays the expected mix of residential redevelopment in } \\
\text { the urban center and classic suburban expansion in all directions on } \\
\text { the metropolitan edge. During the study period development in } \\
\text { Fort Bend County has been significant around Sugarland and } \\
\text { Mission Bend. North Houston also continues on an increasing rate } \\
\text { of residential development. }\end{array}$ \\
\hline $\begin{array}{l}\text { Las Vegas } \\
\text { (Map A.17) }\end{array}$ & $\begin{array}{l}\text { Bounded by mountains on the east, Las Vegas continues to expand } \\
\text { its suburban borders significantly in all other directions into the } \\
\text { surrounding desert. Expansion to the north has been particularly } \\
\text { heavy. }\end{array}$ \\
\hline $\begin{array}{l}\text { Los Angeles } \\
\text { (Map A.18) }\end{array}$ & $\begin{array}{l}\text { The Riverside County portion of the Los Angeles metro area } \\
\text { continues significant suburban expansion and infill of less- } \\
\text { developed areas. Particularly intensive residential development is } \\
\text { occurring in the Murrieta-Temecula-Hemet area of the county and } \\
\text { along the Chino Hills. }\end{array}$ \\
\hline
\end{tabular}




\begin{tabular}{|l|l|}
\hline \multicolumn{1}{|c|}{$\begin{array}{c}\text { Metropolitan } \\
\text { Region }\end{array}$} & \multicolumn{1}{c|}{ Exurban Characterization } \\
\hline $\begin{array}{l}\text { Northern Virginia } \\
\text { (Map A.19) }\end{array}$ & $\begin{array}{l}\text { Significant redevelopment in the vicinity of the new Convention } \\
\text { Center in Washington, D.C., and lesser such activity in the Adams } \\
\text { Morgan and Silver Spring areas show up for the tightly packed } \\
\text { census tracts of the D.C. area. Continued suburban expansion on } \\
\text { the fringes of the greater Washington metropolitan area is evident. } \\
\text { Of note, there are census tracts somewhat equidistant from the } \\
\text { southern fringes of the Washington metropolitan region and the } \\
\text { northern edge of the Richmond area that are developing into } \\
\text { bedroom communities to either city. }\end{array}$ \\
\hline $\begin{array}{l}\text { Phoenix } \\
\text { (Map A.20) }\end{array}$ & $\begin{array}{l}\text { Phoenix continues rapid new residential development to its west } \\
\text { and south, with heavy infill of tracts that have been previously leap- } \\
\text { frogged by developers. The upscale golf course community of Rio } \\
\text { Verde, northwest of Phoenix is a truly exurban development, quite } \\
\text { isolated from the rest of the Phoenix area. }\end{array}$ \\
\hline $\begin{array}{l}\text { San Francisco Bay } \\
\text { Area } \\
\text { (Map A.21) }\end{array}$ & $\begin{array}{l}\text { California. The Ladoga-Leesville area of Colusa County has } \\
\text { sprouted new housing developments in undeveloped areas that } \\
\text { would be isolated alternatives to living in the Sacramento area, } \\
\text { other Central Valley cities in the Interstate 5 corridor, or the North } \\
\text { Bay. Suburban expansion is evident all through the Central Valley- } \\
\text { extending into the foothills of the Sierra Nevada mountains. } \\
\text { Significant farmland conversion to residential communities } \\
\text { continues in Sacramento County in the region between the outskirts } \\
\text { of Sacramento and the Sacramento Airport. }\end{array}$ \\
\hline
\end{tabular}

When viewed on the maps, results of the ex ante classification were mixed. It became apparent that while the classification was able to correctly detect significant exurbs, residential redevelopment activities in established urban areas also were flagged as "emergent" exurbs. This occurred because redevelopment typically converts land from commercial or industrial use to intensive residential - giving the appearance of a new isolated residential area. These tracts appear as relatively small and tightly-packed census tracts within the established cities on the maps. Truly exurban developments appear as tracts covering very large areas - beyond the established cities on the maps.

The classification did the best job of identifying suburban development. In some cases, smaller redevelopment activities or minor residential infill was misclassified as suburban development, but for the most part, the suburban development was found to occur on the fringes of existing metropolitan areas in geographically large census tracts. Large census tracts are an indication that population was relatively sparse in that area at the time of the last Census. We suggest adding a control for the geographic area of the tract to perhaps distinguish between urban redevelopment and exurban development in the identification of emergent developments.

\section{Single-Family Home Purchase Geographic Trends}


The HMDA data were summarized at the census tract level to determine the change in home purchases during the study period. Map A.22 illustrates the average annual number of single-family home purchases by census tract during the study period. As would be expected, higher averages cluster around metropolitan areas and other urban centers, while rural areas show much more sparse home buying activity. Map A.23 illustrates the significant and widespread growth in home buying during the study period. Of interest, many areas of the country show declines in home purchasing between 1996 and 2004. Several explanations are possible. First, if build out of a particular tract was peaking in the late 1990's, a drop in home buying would be expected in the early part of the current decade. Second, "hot" real estate markets in some metropolitan areas may have cooled off in response to economic conditions. Finally, because the map displays all negative changes in home buying as red, even very slight decreases in home buying are lumped in with those that could be expected under the two previous cases described.

Another key geography-dependent result is the distribution of average home values. This distribution for 2004 appears on Map A.24. Average home value by census tract has been segmented fairly coarsely to illustrate geographic differences more clearly. The national average home value, based on the 2004 HMDA data, was $\$ 178,900$. However, relatively little of the country, geographically, approaches this average. The vast majority of land area in the country has values that fall well below the national average.

Results on home values lead to results on affordability. Map A.25 presents the 2004 countylevel median family income geographically. Recall that the 2004 national median was $\$ 57,500$. However, again we see that the vast majority of the counties in the country have median incomes below the national average. In general, only the largest metropolitan areas have median incomes above the national average. These two maps, A.22 and A.23 illustrate one aspect of the much-studied urban-rural divide.

Drilling a little deeper, we overlaid home buyer income change with home value change in Maps A.26 and A.27. This was done only for urban tracts that were not redefined as part of the 2000 Census to avoid redefinition issues. Consequently a few key metropolitan centers like Seattle, Houston, and Milwaukee do not appear. Map A.26 illustrates how incomes perform compared to home values. Map A.27 shows how home buyer incomes and home values jointly perform compared to the area median family income. Map A.27 may be the more interesting of the two. In general, it appears that both income and home value are outpacing median income growth. This suggests that healthy amounts of lower wage jobs are dampening growth in median income, while higher paid home buyers are seeing their incomes and home values rise in tandem. The opposite appears to be true in Portland, Oregon, and Indianapolis. Home values and home buyer incomes are not keeping pace with growth in median income. More investigation is needed to determine whether the cause in both locations might be high-income renters or perhaps some slowing of in-migration from mid-1990's levels.

\section{Metropolitan Census Tracts}


Results have been plotted for the specific high-growth metropolitan areas illustrated in Maps A.28-32. The following metropolitan areas or Census Bureau Statistical Areas (CBSAs) were selected to be geographically representative of high-growth in home buying activity during the study period. These 12 areas appear on the 11-window maps in Maps A.28-32. Portions of Los Angeles and Riverside CBSAs appear in the same map window.

- Atlanta - Sandy Springs - Marietta, GA

- Denver - Aurora, CO

- Houston - Baytown - Sugar Land, TX

- Las Vegas - Paradise, NV

- Los Angeles - Long Beach - Glendale, CA

- Minneapolis - St. Paul - Bloomington, MN

- New York - Wayne - White Plains, NY-NJ

- Phoenix - Mesa - Scottsdale, AZ

- Portland - Vancouver - Beaverton, OR-WA

- Riverside - San Bernardino - Ontario, CA

- Tampa - St. Petersburg - Clearwater, FL

- Washington - Arlington - Alexandria, DC-VA-MD-WV

These metropolitan maps need to be used or otherwise read together to begin to derive meaningful analysis of dynamic market conditions affecting specific metropolitan areas. Of intersest, the maps work well to explain home buying trends in some markets. For example, the maps show cases where there is a visible gradient in average home values and an associated trend in home buying from relatively expensive areas to realtively inexpensive areas. In those cases, rural build-out in locations where home values are low relative to the rest of the metro area probably is driving the shifting home buying patterns.

The study-period average number of single-family home purchases by tract is depicted in Map A.28. This map provides a good refrerence point for determining relative home buying activity between census tracts, and provided numerical context to subsequent maps indicating percent changes.

The 2004 average home values by tract appear in Map A.29. Using Map A.29 with Map A.30, some interesting results appear. Pronounced shifts in growth patterns from one side of the metro area to another side affect Denver (west to east), Atlanta (north to south), and Phoenix (east to west). These shifts correspond to home value gradients. The shift in home buying in these metro areas has been from relatively high-value areas to relatively low-value areas. This would be consistent with the building out of rural areas where land values may have been relatively low, prior to development.

The differences between major metropolitan areas in their growth patterns are interesting. Based on Map A.30, some areas appear to be growing in relatively uniform fashion, such as the D.C. area, New York, and to some degree the Tampa and Las Vegas areas. Las Vegas is interesting because the map suggests the effects of successive suburban rings of development. The outside edges of Las Vegas are growing rapidly, but just in from the outer edges, home buying activity has slowed or is declining. Significant growth during the study 
area has been occurring in essentially all parts of these metro areas. Houston also provides an interesting study. Home buying activity has been relatively minimal in the tract developments surrounding the urban core of the city, and values are also relatively low. However there is some evidence that activity in the residential core of the city is increasing. This also appears to be happening in the greater Los Angeles metroplex, as well. Relatively light volumes of home buying have been occurring in the oldest neighborhoods, but the rate of activity is increasing steadily.

Los Angeles, Portland, and Minneapolis display attributes of distant suburban development (Map A.30). The trend is similar to Las Vegas' rapidly expanding suburbs, but in these larger metropolitan areas the growth is occurring beyond the portion of the area shown on the map. The home buying rate generally is decreasing in established suburbs and increasing the further out one goes.

The analysis attempted to look at the urban gentrification issue by evaluating the change in home buyer income for urban census tracts during the study period. We screened tracts for those having a real change in average home buyer income greater than 1.5 times the 1996 tract average. These results appear in Map A.31 as degrees of income change exceeding 1.5 times the 1996 tract average. Income growth in the urban core is one recognized indicator of gentrification. That, coupled with increased home buying activity, could suggest areas where urban "mansionization" could be likely. This is one phenomenon of gentrifying neighborhoods where higher income home buyers purchase a modest, older-vintage, home on a modest urban lot and proceed to either radically upsize the home on the same lot via extensive remodeling or they raze the home and build a custom home in its place. Two somewhat recent news stories introduce this trend (Christie 2005 and Risen 2005).

We also analyzed the tract-level single-family home purchase lender market share for the listed metropolitan areas. Such analysis gives the "lay of the land" in terms of which lenders play in these markets, which serve growing suburbs, and which serve urban cores. Several observations are possible by viewing the results that appear in Map A.32. First, for some metro areas, visual inspection provides a quick sense of which lender tier of the market leads the home purchase market. In the case of Los Angeles, Portland, New York, and Minneapolis, one of the lender tiers clearly dominates mortgage activity. Phoenix and Atlanta are interesting because the urban core and close suburban areas are dominated by second-tier lenders while the outer suburbs (faster growing areas) are dominated by third-tier lenders. Denver and Houston show a similar pattern of first- and second-tier lenders leading mortgage activity in the established residential areas of those cities, while third-tier lenders lead in the growing suburban areas. 


\section{DISCUSSION}

Falling mortgage interest rates have spurred widespread expansion of home buying activity in the U.S. Nationally, the annual build out of new housing increased 50 percent during the study period. In the same period, annual mortgage financed home buying increased by over 80 percent. These are significantly large numbers representing housing units, not dollars. The housing boom, as it has been termed commonly, with the associated rise in home values and equity has contributed to a boom in home owner disposable income. As rates have fallen, home buyers can and have demanded more house for the same money or have used increased home equity and stepped up in price range to higher-end homes.

Incomes of home buyers are growing relative to median family income. The national median family income in 2004 was $\$ 57,500$; however that number varies widely by geography as shown on Map A.27. Based on the national median, a significant (hundreds of thousands) and growing proportion of home buyers have incomes in excess of \$172,500 (2004 dollars). More research into the income characteristics of home buyers would result in more specific income segmentation of the market.

Migration to the South, Southwest, and West continues to be very strong. There is also evidence that the Plains and Midwest are on the verge of residential expansion. The Hot-Dry and Hot Humid climate zones have grown the fastest during the study period. The Cold and Mixed Humid climate zones' share of housing activity has declined steadily during the study period.

Government loan products (one tool for achieving energy efficiency improvements in homes) are declining in popularity with lenders. The principle behind energy-efficiency mortgages is that lenders would bundle financing of a package of energy efficient technology options on a new home or improvements on an existing home into the primary mortgage being offered to the home buyer. Low rates have resulted in a move away from government loan products by lenders. This occurs for two reasons in such an environment. First, under low conventional rates, the home buyers previously helped by government loan products are able to qualify for conventional products. Second, in high-volume environments (during low interest rates), lenders favor products that are the quickest to originate, such as their own conventional products.

The tiers of the home purchase lending market are quite varied by geography and surprisingly more regionalized than we expected. First-tier lenders lead the markets of the West Coast, Northern Rockies, and Minnesota. Second tier lenders lead in the Northeast and Florida. Third tier lenders lead in the growth markets of Las Vegas, Phoenix, Dallas, Atlanta, and Raleigh-Durham. Fourth tier lenders dominate rural America and cover the greatest geographic expanse.

\section{Geographic Display of Results}


Spatial display of geographic results challenges the best analysts. There are information value trade-offs that must be made. Viewing results graphically on a map logically would seem preferable to tabular presentations. The use of color and geographic references adds information value to spatially significant results otherwise presented in tabular form.

Trend information is more difficult to view using maps. Color variations must represent changes over a time period. Individual steps in a trend can only be displayed using multiples of each map corresponding to the time steps of the analysis. In addition, to combine measures for more effective spatial analysis, the reader may need to refer to two to three maps simultaneously.

\section{$\underline{\text { Study Limitations }}$}

We acknowledge several aspects of the study that could be improved given more time and resources.

- Developing linkage to the 2000 Census microdata from the long form household survey would provide helpful linkage to household management affecting residential energy use. It might also be used to illuminate inevitable differences between family incomes in general and those of home buyers for the same geography. The data quality issues with vendor-customized census data were significant enough to require too much time and expense to facilitate incorporation of those data into this report.

- No research was done to establish the effect of down payments on the mortgage amounts reported in the HMDA data. As stated, we assumed that in the whole of all mortgage transactions, the effect of some borrowers making large down payments would have minimal impact on average values. We can offer no evidence that this assumption is reasonable other than general knowledge of industry practice. Because we have extended the assumption to suggest that mortgage amounts serve as a reasonable proxy for actual home sales prices, care should be taken that perhaps home values reported could be understated by some unknown amount.

- Income data or geographic data were missing for a nontrivial amount (6\%-10\%) of the HMDA records in all years of the analysis. Missing either of these data for any one mortgage record invalidates that record for median income analysis. Both an income and a valid geographic code (to at least the county) are required. Improper geocoding by the submitting institutions causes the bulk of this problem with the data.

- Broad brush studies, by definition, avoid depth that some analysts would appreciate. We welcome suggestions on more in-depth studies that might follow from this study. Several examples of potential extensions of this work are provided below.

\section{CONCLUSION}

The report is intended to provide market information to technology research managers addressing the residential sector. The trends presented should help inform decisions about how best to pursue energy efficiency with residential home buyers. At a minimum, gaining 
an understanding of the information here should provide rich background and context for planning technology research in the future.

\section{$\underline{\text { Key Findings }}$}

- The share of home building and home buying activity continues to rise steadily in the Hot-Dry and Hot-Humid climate zones, while the Mixed-Humid and Cold climate zone shares continue to decline. Other zones remain relatively stable in terms of share of housing activity.

- The proportion of home buyers having three times the median family income for their geography has been steadily increasing during the study period.

- Growth in the Latino population and to a lesser degree in the Asian population has translated into proportional increases in share of home purchasing by both groups. White home buyers continue to decline as a proportion of all home buyers.

- Low interest rate climate resulted in lenders moving back to conventional financing, as opposed to government-backed financing, for cases that would be harder to finance in higher rate environments. Government loan products are one mechanism for affecting energy efficiency gains in the residential sector.

- The home finance industry quartiles (2004) feature 5 lenders making up the first quartile of home purchase loans, 18 lenders making up the second quartile, 111 lenders making up the third quartile, and the remaining nearly 8,000 lenders make up the fourth quartile.

- We explored the emergence of extra-urban or "exurb" development patterns in several major metropolitan areas. The mortgage data can be used to identify new "islands" of residential development, but effectively classifying these islands as exurban needs additional effort.

- We illustrated the issue of subprime mortgage lending and its implications for federal programs seeking to partner with mortgage lenders. Federal programs seeking to partner with residential lenders need to do their homework on the lending performance of potential partners. Rich public data exist on this topic.

\section{$\underline{\text { Areas Needing Further Research }}$}

The BT portfolio of R\&D programs (and any R\&D portfolio addressing the residential sector), looking out to 2025, needs to consider the impact of the changing landscape of home ownership to ensure its program is correctly targeted and positioned, given expected changes in the market place. Several factors influencing demographic shifts in the U.S. also affect the demand for residences. These include racial and cultural shifts, migration, architectural considerations, wealth, and the interest rate environment.

Because this analysis has provided broad coverage of the national home buying market, little depth into some specific issues has been provided in this report. The following areas constitute suggestions for addition research that could follow from this study.

- With improved projections of heating and cooling in particular, we can weigh the cost-effectiveness of regional investment in residential energy efficiency with 
comparable investments in electricity and natural gas infrastructure and help determine where new generation, transmission, and distribution capacity may be avoided.

- Using the HMDA data, we can perform detailed lender performance analysis of DOE-partner lenders, Building America development lenders, or prospective residential lending partners. Such analysis can include low-income and minority lending performance, subprime lending performance, geographic segmentation, and comparison to industry peers in all cases.

- Much more depth is possible in the area of home buyer income analysis. We can develop income-based market segments and perform detailed market analysis on each segment. This could include lender peer performance analysis, race-by-income analysis, and loan amount-income analysis.

- Racial demographics of home buyers can be analyzed in much greater detail - similar to the income analyses suggested above.

- Greater spatial detail and segmentation of specific results is possible. In addition to the national display of detailed geographic results, we can perform analyses at the state, county, congressional district, or any other custom spatial aggregation.

- The current study period ends at 2004 due to the availability of the HMDA data. As subsequent years are released, these results should be updated and extended. 


\section{REFERENCES}

Briggs, Robert S., Z. Todd Taylor, and Robert G. Lucas, 2002a. "Climate Classification for Building Energy Codes and Standards: Part 1 -- Development Process." ASHRAE Transactions 2002, V. 109, Pt. 1.

Briggs, Robert S., Z. Todd Taylor, and Robert G. Lucas, 2002b. "Climate Classification for Building Energy Codes and Standards: Part 2 -- Zone Definitions, Maps, and Comparisons." ASHRAE Transactions 2002, V. 109, Pt. 1.

Christie, Les, 2005. “Die, Die, Monster Home! Die!”, CNNMoney,com, August 18, 2005. Accessed online on June 8, 2006:

http://money.cnn.com/2005/08/18/real_estate/monster_home_backlash/index.htm

EERE-BTP 2006. Building America: Research Project Locations, Project website and database accessed online on 11/5/2005:

http://www.eere.energy.gov/buildings/building_america/cfm/project_locations.cfm.

Davis, Morris A. and Jonathan Heathcote, 2004. "The Price and Quantity of Residential Land in the United States," Finance and Economics Discussion Series 2004-37, Federal Reserve Board 2004. Accessed online on March 20, 2006:

http:/www.federalreserve.gov/pubs/feds/2004/200437/200437pap.pdf

FFIEC 1997. 1996 Loan Application Register (LAR) \& Transmittal Sheet (TS) Raw Data on CD-ROM, Federal Financial Institutions Examination Council, Washington DC.

FFIEC 1998. 1997 Loan Application Register (LAR) \& Transmittal Sheet (TS) Raw Data on CD-ROM, Federal Financial Institutions Examination Council, Washington DC.

FFIEC 1999. 1998 Loan Application Register (LAR) \& Transmittal Sheet (TS) Raw Data on CD-ROM, Federal Financial Institutions Examination Council, Washington DC.

FFIEC 2000. 1999 Loan Application Register (LAR) \& Transmittal Sheet (TS) Raw Data on CD-ROM, Federal Financial Institutions Examination Council, Washington DC.

FFIEC 2001. 2000 Loan Application Register (LAR) \& Transmittal Sheet (TS) Raw Data on CD-ROM, Federal Financial Institutions Examination Council, Washington DC.

FFIEC 2002. 2001 Loan Application Register (LAR) \& Transmittal Sheet (TS) Raw Data on CD-ROM, Federal Financial Institutions Examination Council, Washington DC.

FFIEC 2003. 2002 Loan Application Register (LAR) \& Transmittal Sheet (TS) Raw Data on CD-ROM, Federal Financial Institutions Examination Council, Washington DC.

FFIEC 2004. 2003 Loan Application Register (LAR) \& Transmittal Sheet (TS) Raw Data on CD-ROM, Federal Financial Institutions Examination Council, Washington DC. 
FFIEC 2005. 2004 Loan Application Register (LAR) \& Transmittal Sheet (TS) Raw Data on CD-ROM, Federal Financial Institutions Examination Council, Washington DC.

FFIEC 2005a. 2004 Guide to HMDA Reporting, Federal Financial Institutions Examination Council, Washington DC. Accessed online on March 31, 2006:

http://www.ffiec.gov/hmda/pdf/2004guide.pdf.

Federal Reserve System (FRS) 2006. National Information Center website. Financial data and institution characteristics collected by the Federal Reserve System. Accessed online during Jan., Feb., Mar. 2006: http://www.ffiec.gov/nicpubweb/nicweb/nichome.aspx.

Federal Trade Commission (FTC) 2006. Early Termination Notices under Pre-Merger, HartScott-Rodino Act. Accessed online during Jan., Feb., Mar. 2006:

http://www.ftc.gov/bc/earlyterm/.

Freddie Mac 2006. Primary Mortgage Market Survey Archives, accessed online on February 10, 2006: http://www.freddiemac.com/pmms/pmms archives.html.

Lexis-Nexis 2006. Corporate Affiliations.com, website indicating mergers and acquisitions activity. Accessed online during Jan., Feb., Mar. 2006:

http://www.corporateaffiliations.com/executable/cn mergers.asp.

Mortgage Mag 2006. Mortgage Magazine Online. Accessed online during Jan., Feb., Mar. 2006: http://www.mortgagemag.com/.

Risen, Clay, 2005. "McMansion Meets SoHo: Suburbanites' Penchant for Urban Lofts", Slate.com, February 16, 2005. Accessed online on June 8, 2006:

http://www.slate.com/id/2113614/

U.S. Census Bureau 1997. 1996 Annual Housing Units Authorized by Building Permits, U.S. Census Bureau, Washington D.C. Data provided via email on July 28, 2005.

U.S. Census Bureau 1998. 1997 Annual Housing Units Authorized by Building Permits, U.S. Census Bureau, Washington D.C. Data provided via email on July 28, 2005.

U.S. Census Bureau 1999. 1998 Annual Housing Units Authorized by Building Permits, U.S. Census Bureau, Washington D.C. Data provided via email on July 28, 2005.

U.S. Census Bureau 2000. 1999 Annual Housing Units Authorized by Building Permits, U.S. Census Bureau, Washington D.C. Data provided via email on July 28, 2005.

U.S. Census Bureau 2001. 2000 Annual Housing Units Authorized by Building Permits, U.S. Census Bureau, Washington D.C. Data provided via email on July 28, 2005.

U.S. Census Bureau 2002. 2001 Annual Housing Units Authorized by Building Permits, U.S. Census Bureau, Washington D.C. Data provided via email on July 28, 2005. 
U.S. Census Bureau 2003. 2002 Annual Housing Units Authorized by Building Permits, U.S. Census Bureau, Washington D.C. Data provided via email on July 28, 2005.

U.S. Census Bureau 2004. 2003 Annual Housing Units Authorized by Building Permits, U.S. Census Bureau, Washington D.C. Data provided via email on July 28, 2005.

U.S. Census Bureau 2005. 2004 Annual Housing Units Authorized by Building Permits, U.S. Census Bureau, Washington D.C. Data provided via email on July 28, 2005. 
Map Appendix

\author{
PNNL-15925 \\ DM Anderson \\ DB Elliott
}




\section{$\underline{\text { Spatial Analysis Presentation }}$}

This appendix contains the maps cited in the report text. All the maps developed for this report were generated using ArcGIS 9.0, the recognized industry standard in geographic information systems. Specific data coverages or other additional information may be made available for future analyses by consulting with the authors.

There are essentially three types of maps used in the report. These include county-level national maps, census tract-level national maps, and census tract-level regional maps. The analysis of the building permit data is presented on county-level maps because that is the resolution of the data. This also applies to the HUD median income data. The mortgage data are available at the census tract level of geographic detail; however for some analyses, such as the lender market share analysis, the data have been aggregated for display at the county level. Market share information is not really meaningful below the county level. We also include a state-level map of lender market share. Census tractlevel maps are useful for seeing the finest level of resolution in the mortgage data. County, state, and national averages cannot accurately reflect the spatial diversity available by seeing results at the census tract.

There are issues with census tract resolution displayed at the national level that become apparent by examining the national census tract maps in this appendix. Census tracts are developed based on the location of groups of the population. Therefore, where population is more densely housed, census tracts are smaller geographically. This presents a problem for national level census tract mapping that can lead to misinterpretation of map data. Urban census tracts are more densely packed together as would be expected for large cities having relatively large amounts of multifamily housing or otherwise densely packed residential neighborhoods. These tracts are barely distinguishable on a national map, while sparsely populated rural tracts are, by definition, much larger and easily seen on a national map. The map reader's eye might lead to placing undue emphasis on rural census tracts at the expense of urban tracts when viewed on a national map. County-level maps mitigate this visual conflict to some degree, because counties are not sized based on population. Tract-level maps are quite valuable for metropolitan level visual analysis, however.

For those interested in the specific geographic projections used in the GIS analysis, we provide the following documentation. The national level maps are presented using the North American Albers Equal Area Conic projection at a scale of 1:16 million. The Alaska inset is presented using the North American Datum 1983 (NAD 83) State Plane Alaska 1 FIPS 5001 projection at a scale of 1:60 million. The Hawaii inset is presented using the NAD 83 State Plane Hawaii 1 FIPS 5101 projection at a scale of 1:13.5 million. The maps of the exurban extent analysis are simply zooms of the North American Albers Equal Area Conic projection. The other metropolitan-level maps are presented using the Mercator projection at scales ranging from 1:1 million to 1:2 million. 


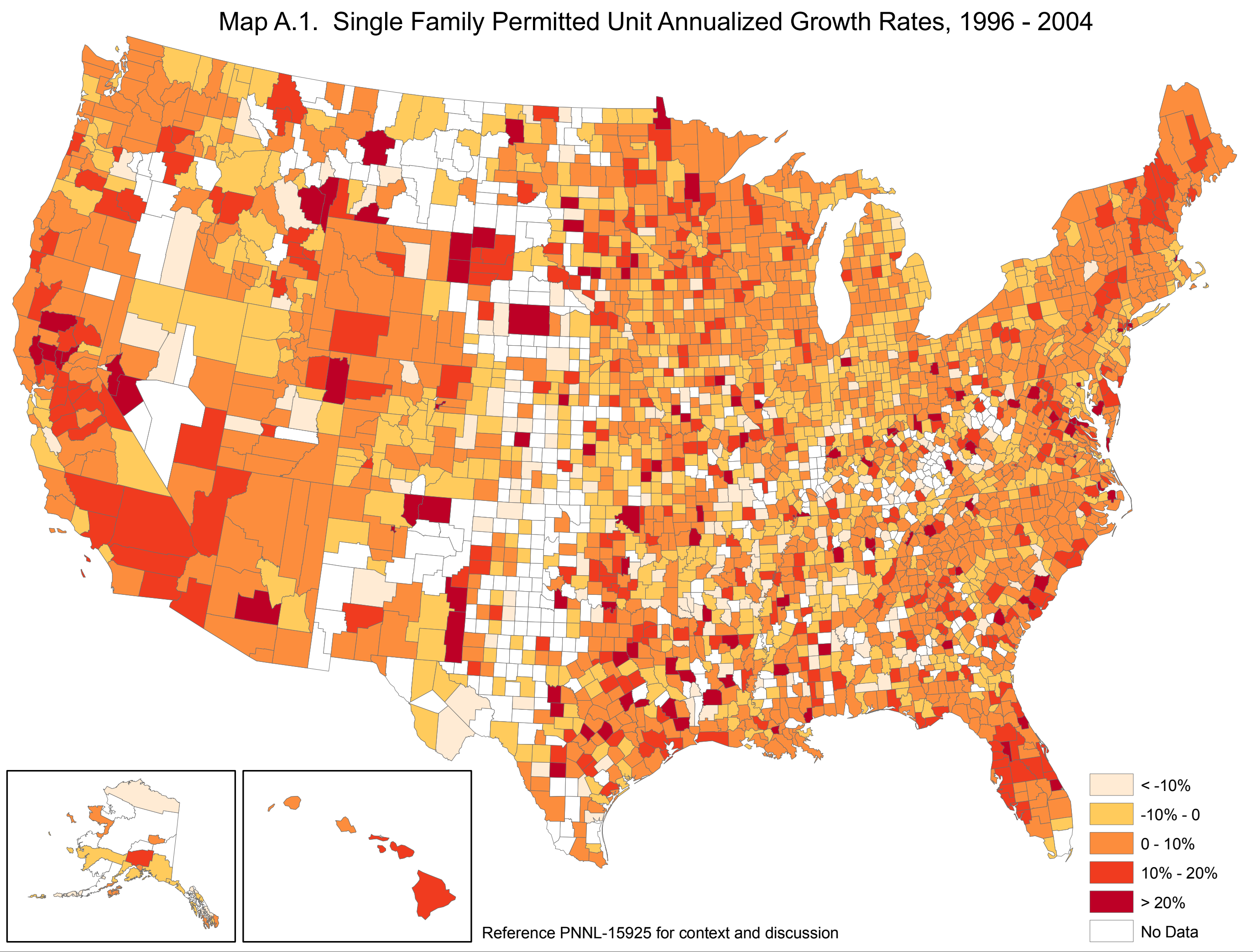


Map A.2. Single Family Permitted Unit Annualized Growth Rates, 2000 - 2004

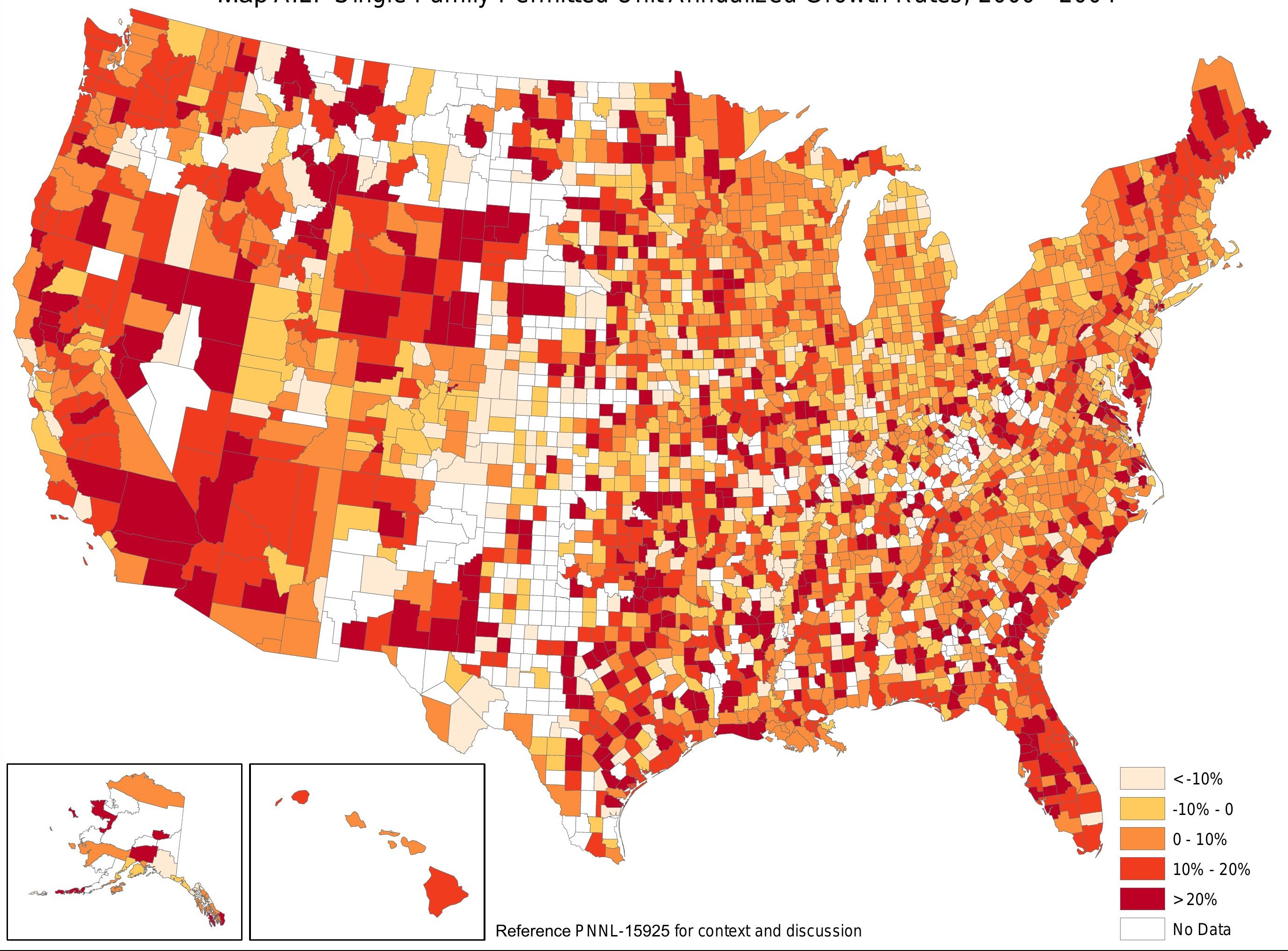


Map A.3. Single Family Permitted Valuation Annualized Growth Rates, 1996 - 2004

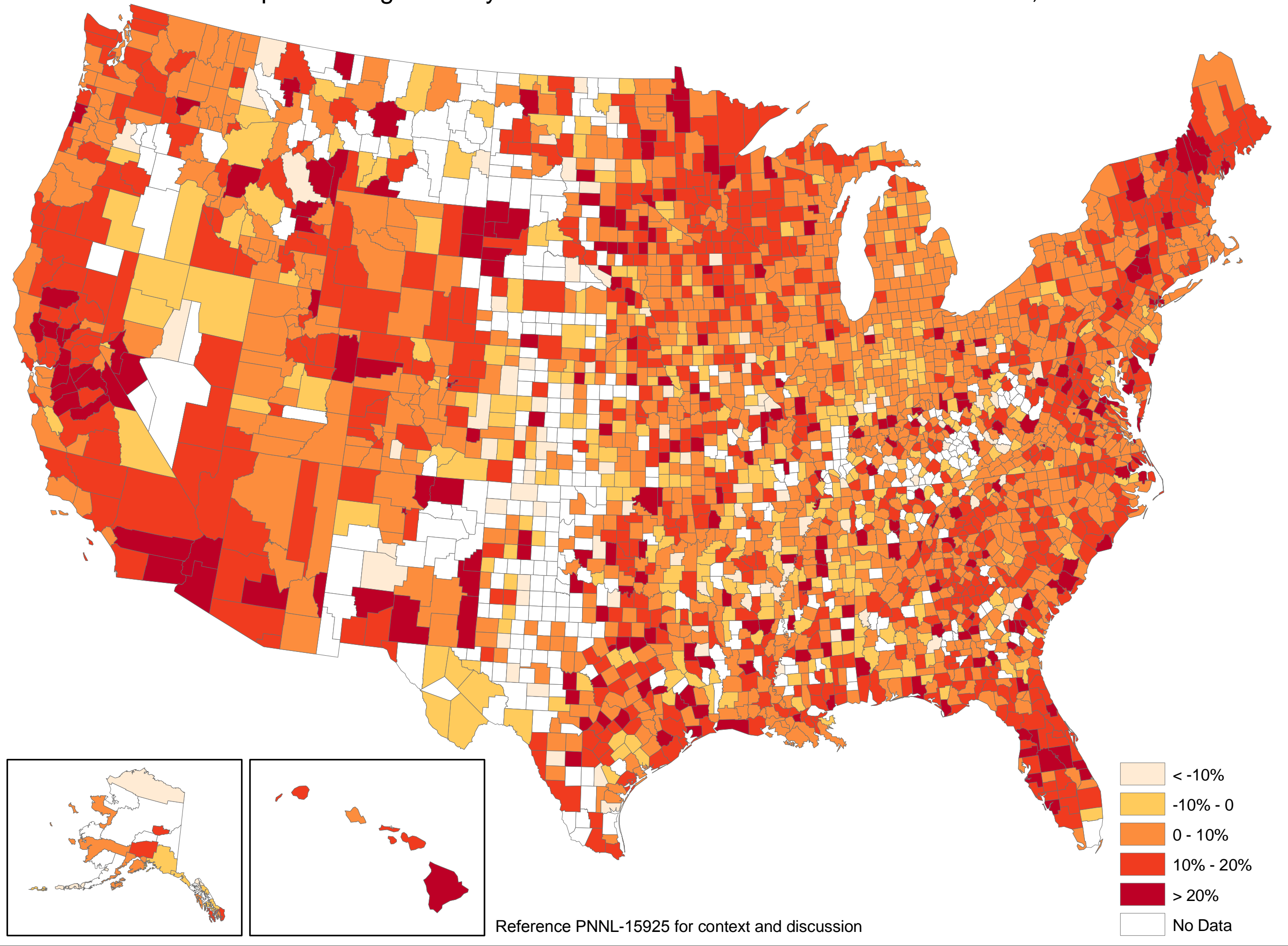


Map A.4. Single Family Permitted Valuation Annualized Growth Rates, 2000 - 2004









Map A.7. Single Family Permitted Unit Absolute Growth, 1996 - 2004

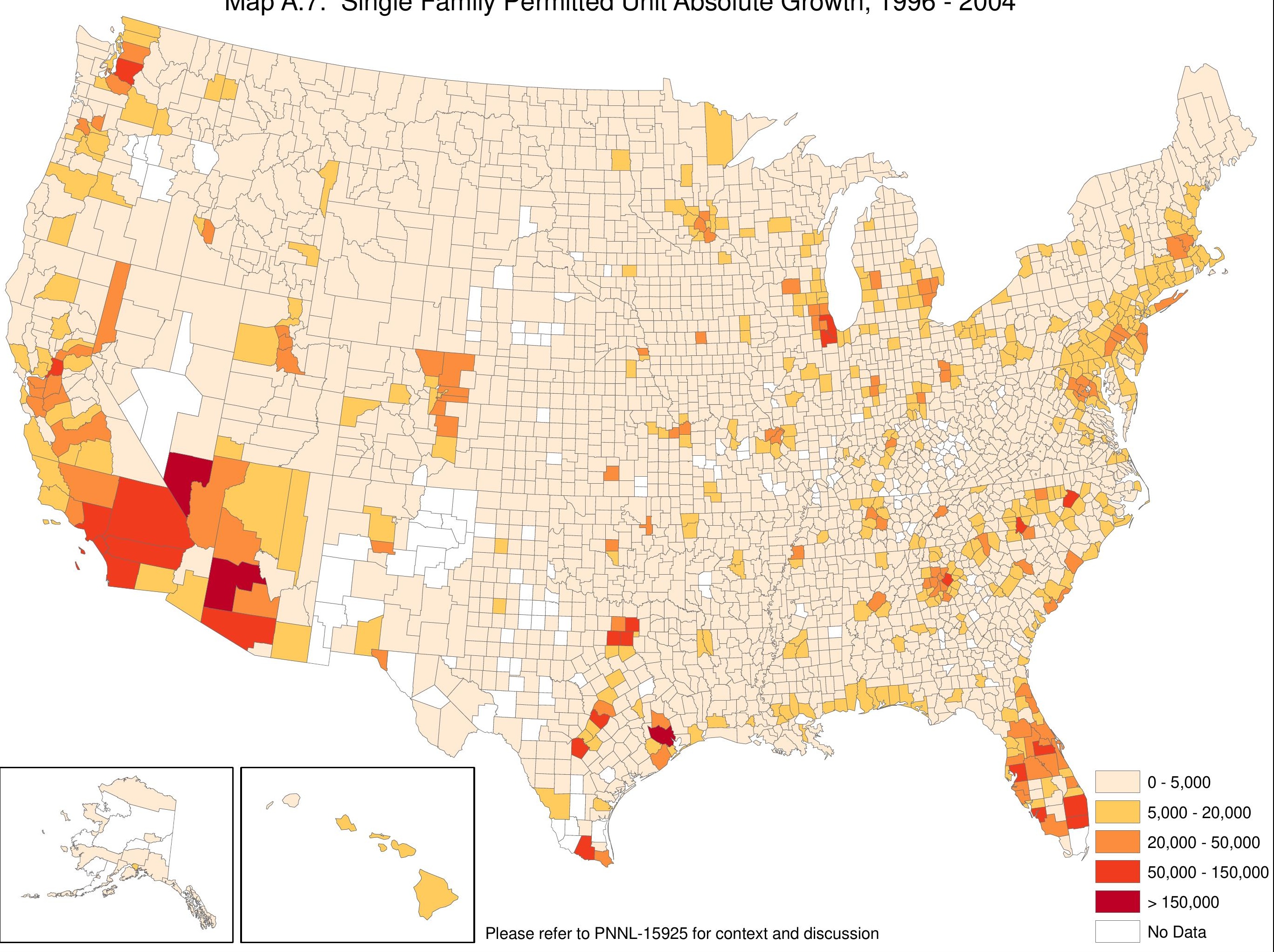


Map A.8. Single Family Permitted Unit Absolute Growth, 2000 - 2004

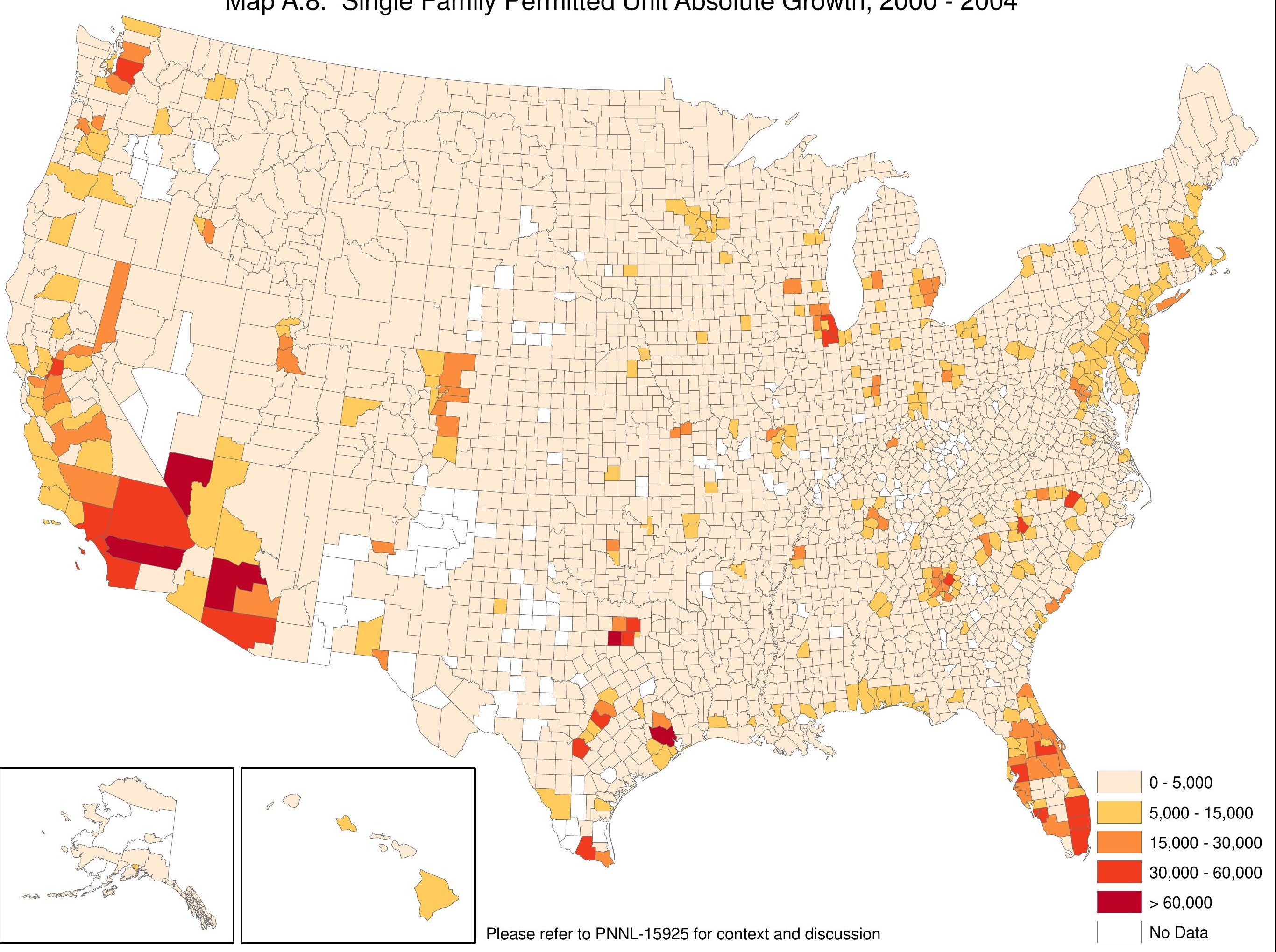




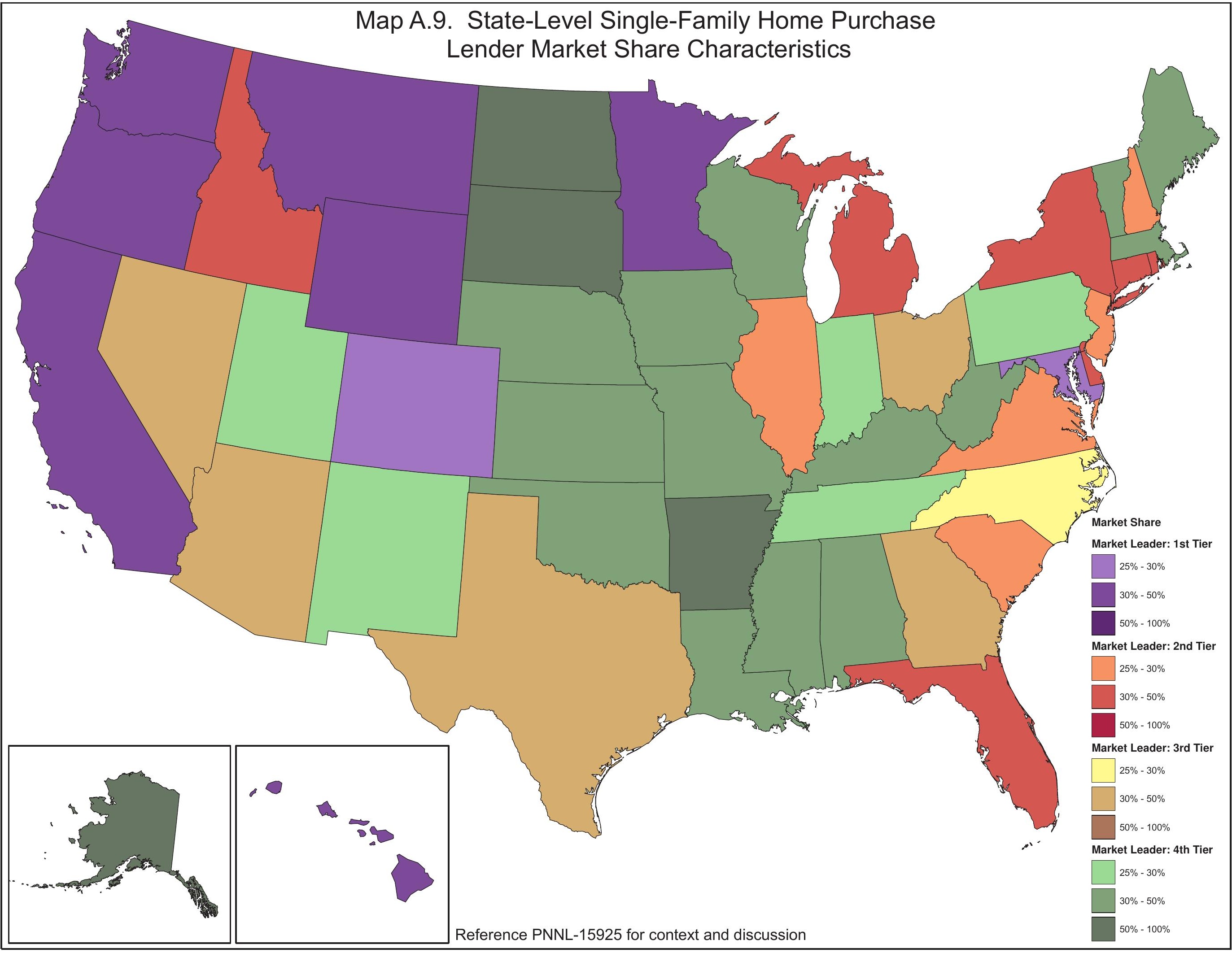




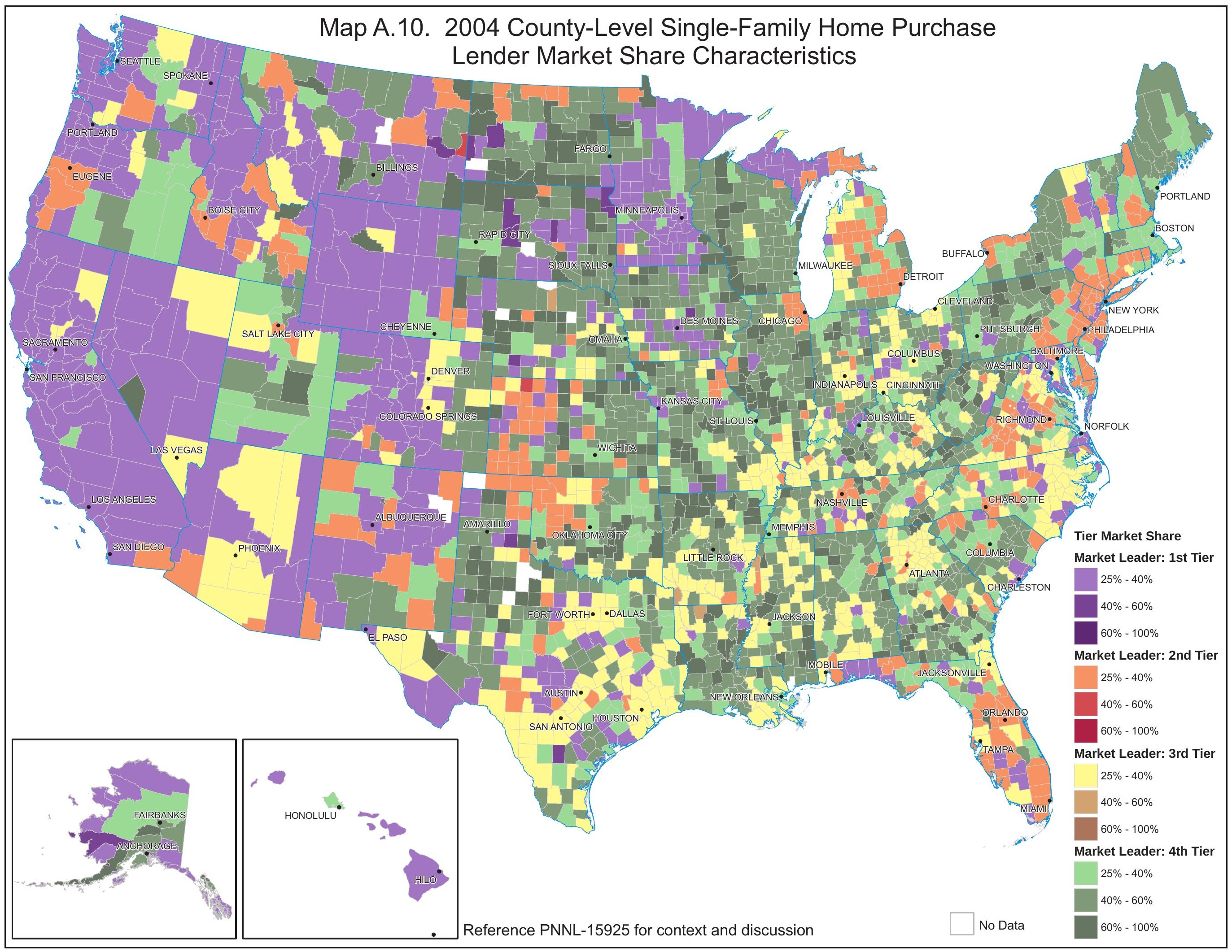




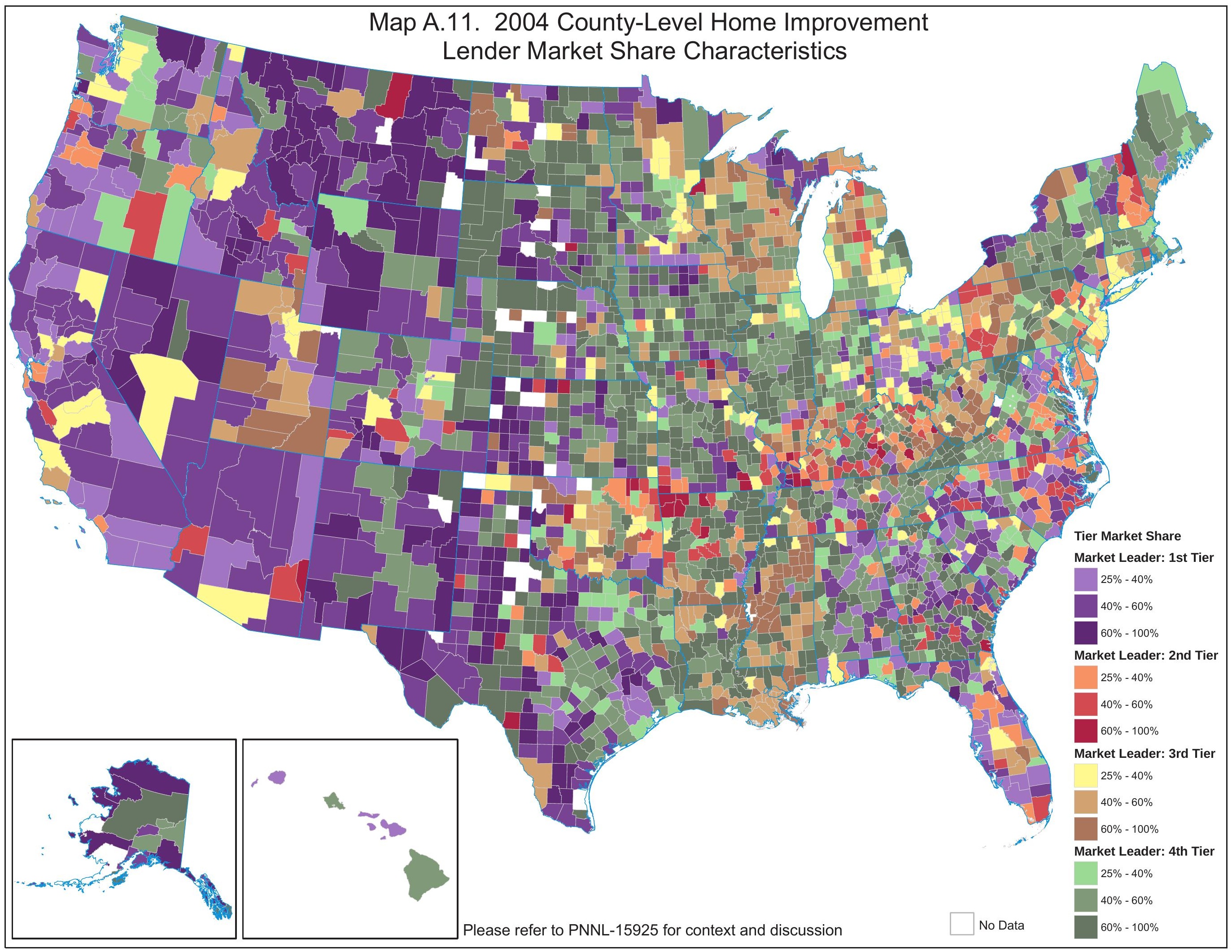




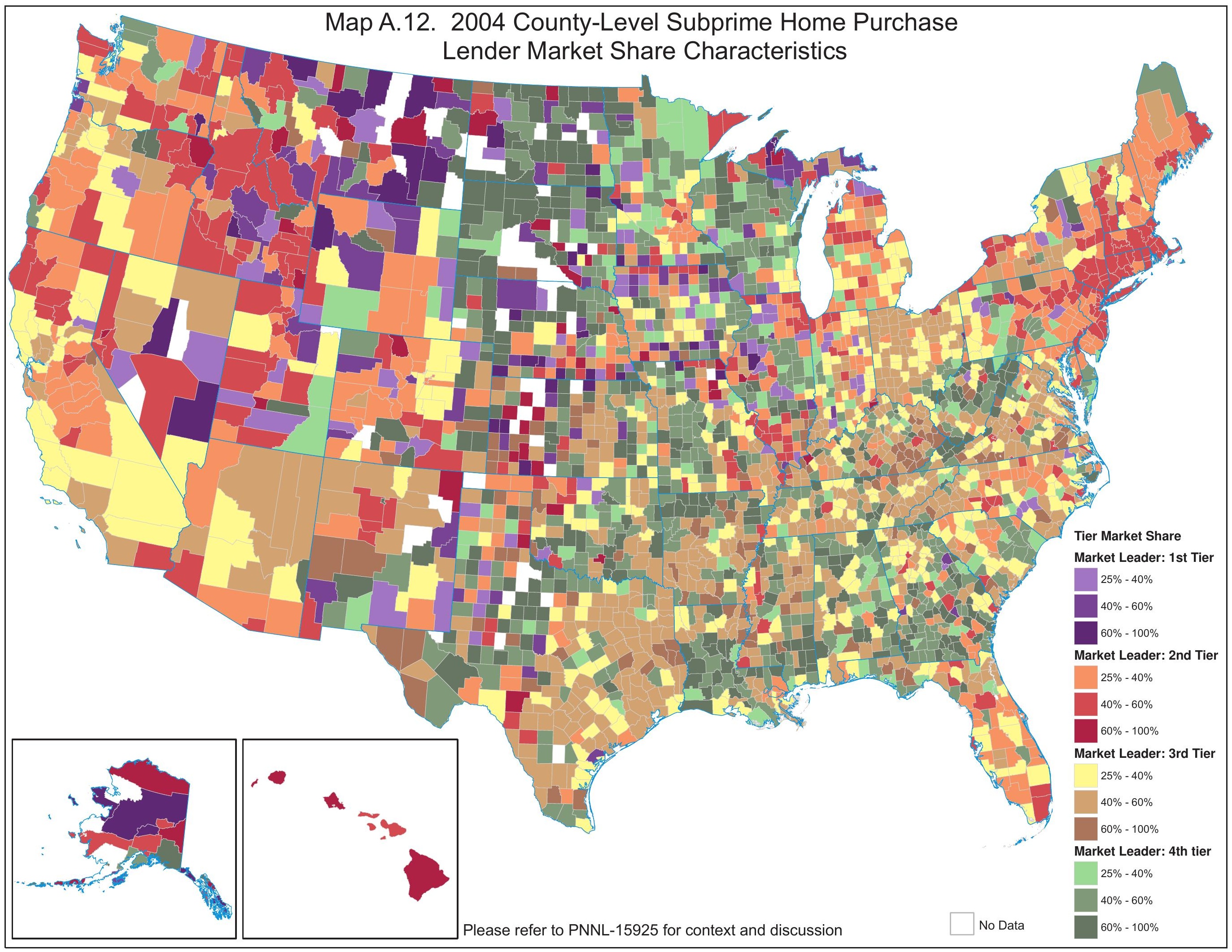




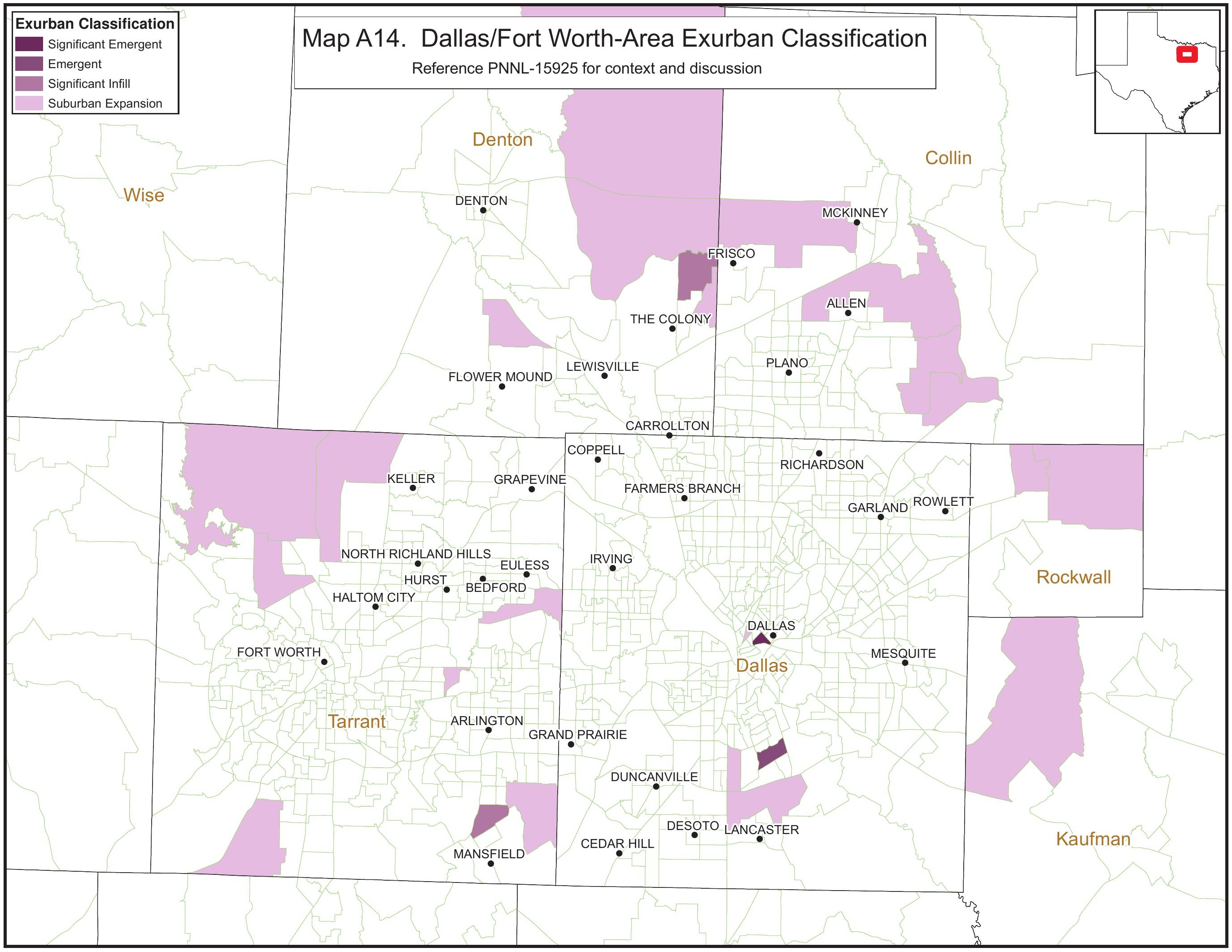

\section{$\sqrt{\text { Exurban Classification }}$}

Significant Emergent

Significant Infill

Suburban Expansion

Wise

\section{Map A14. Dallas/Fort Worth-Area Exurban Classification}

Reference PNNL-15925 for context and discussion

$$
\text { GARL }
$$

Rockwall 


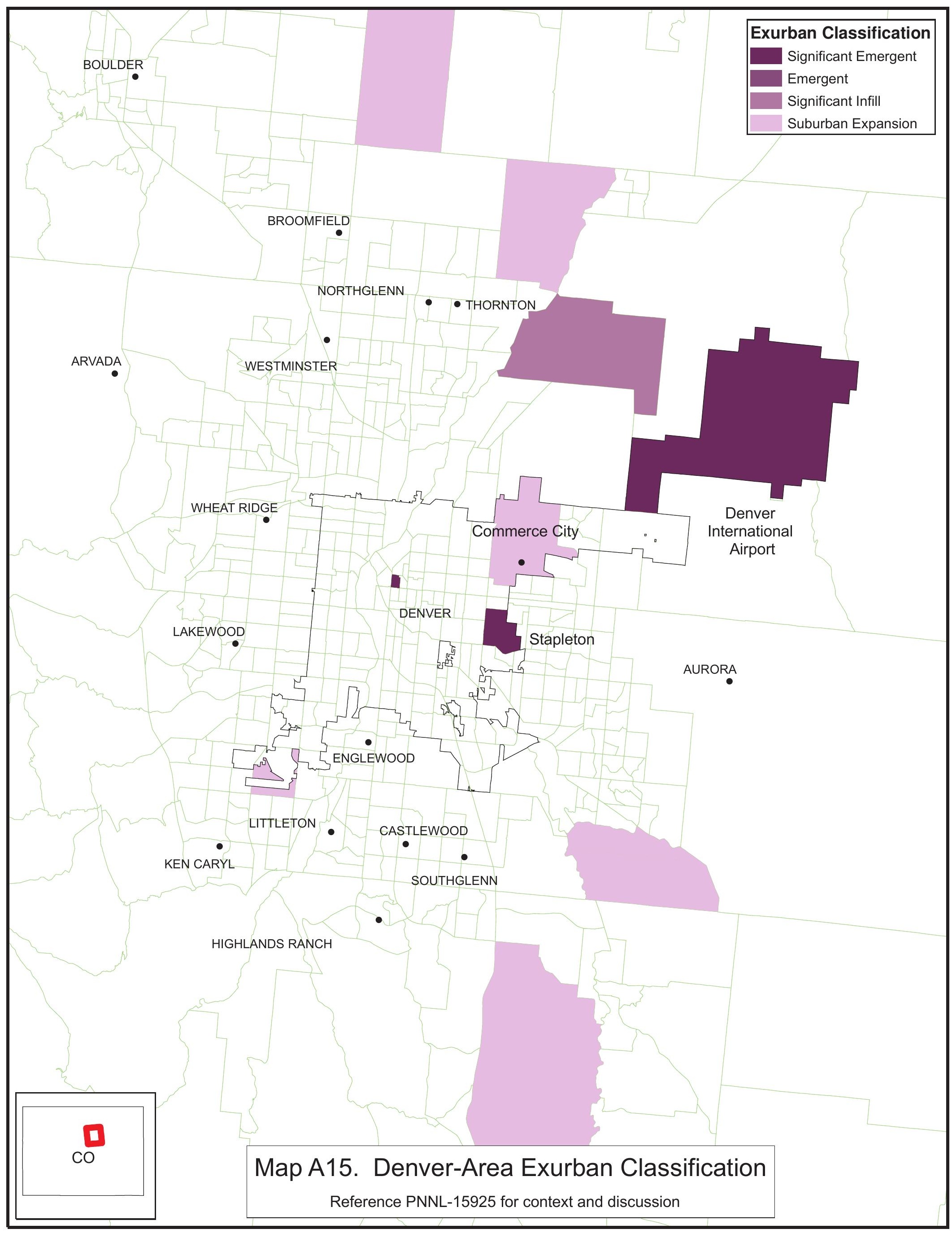


Exurban Classification

Significant Emergent

Emergent

Significant Infill

Suburban Expansion

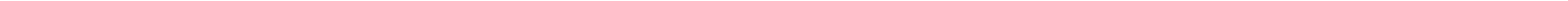




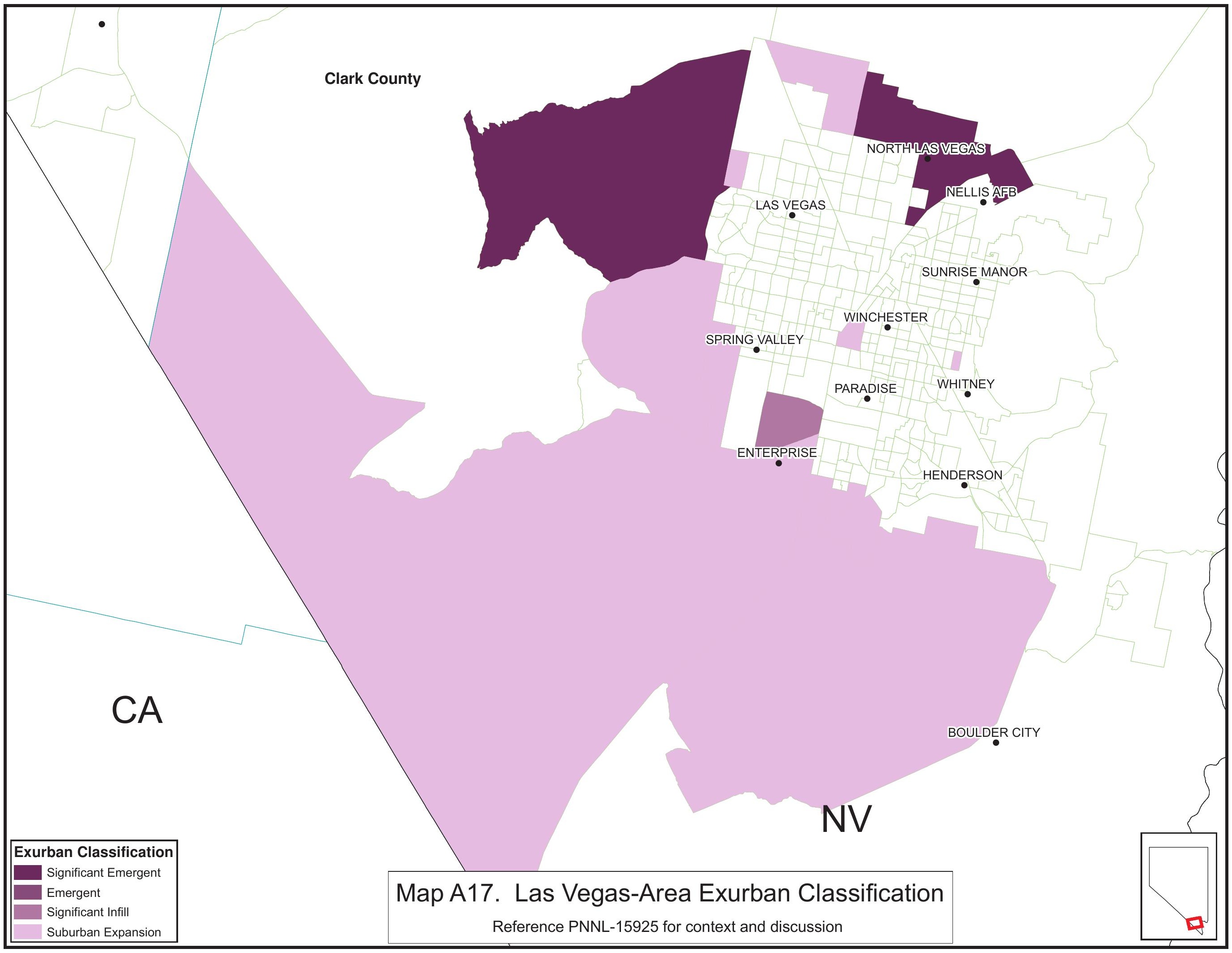




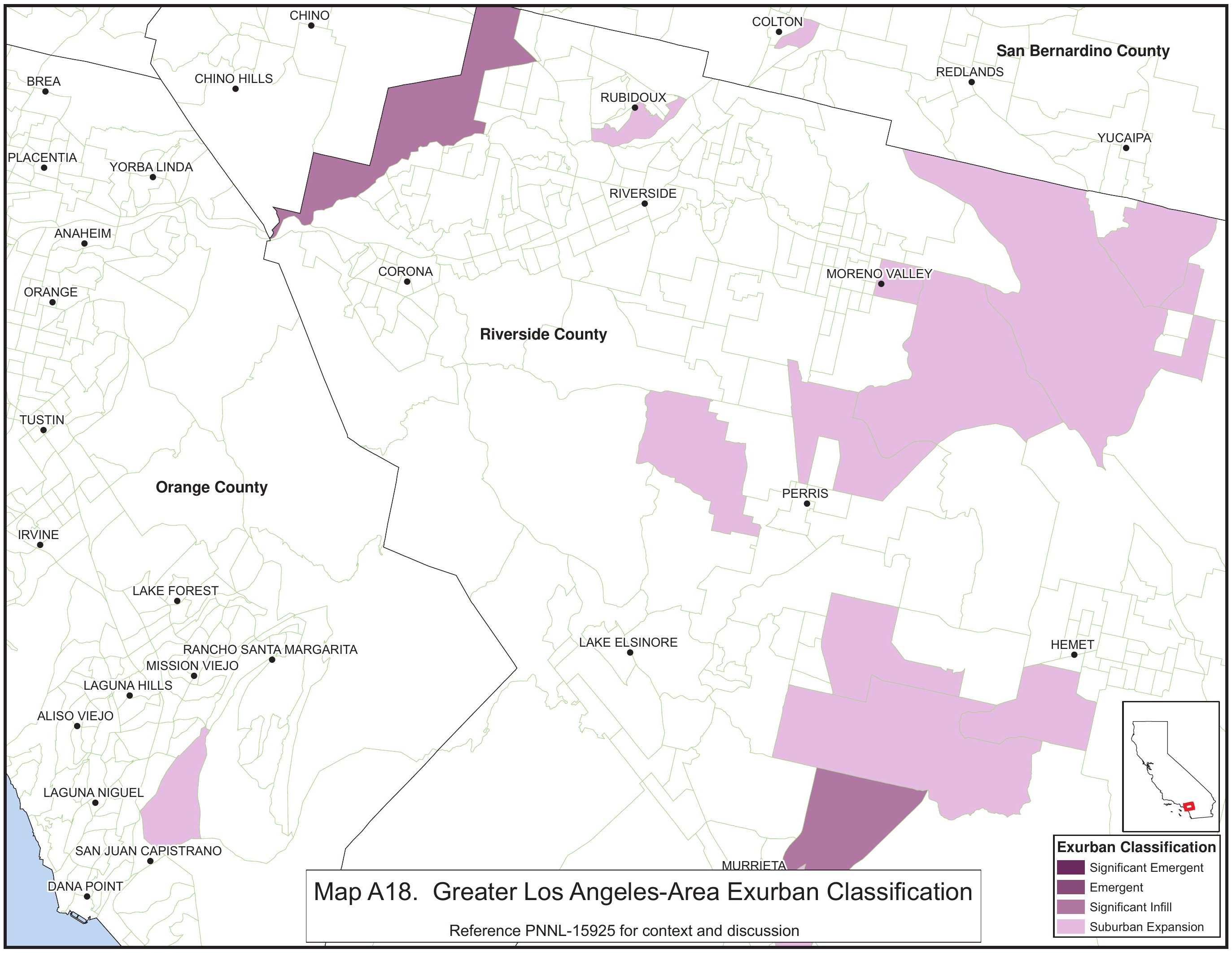




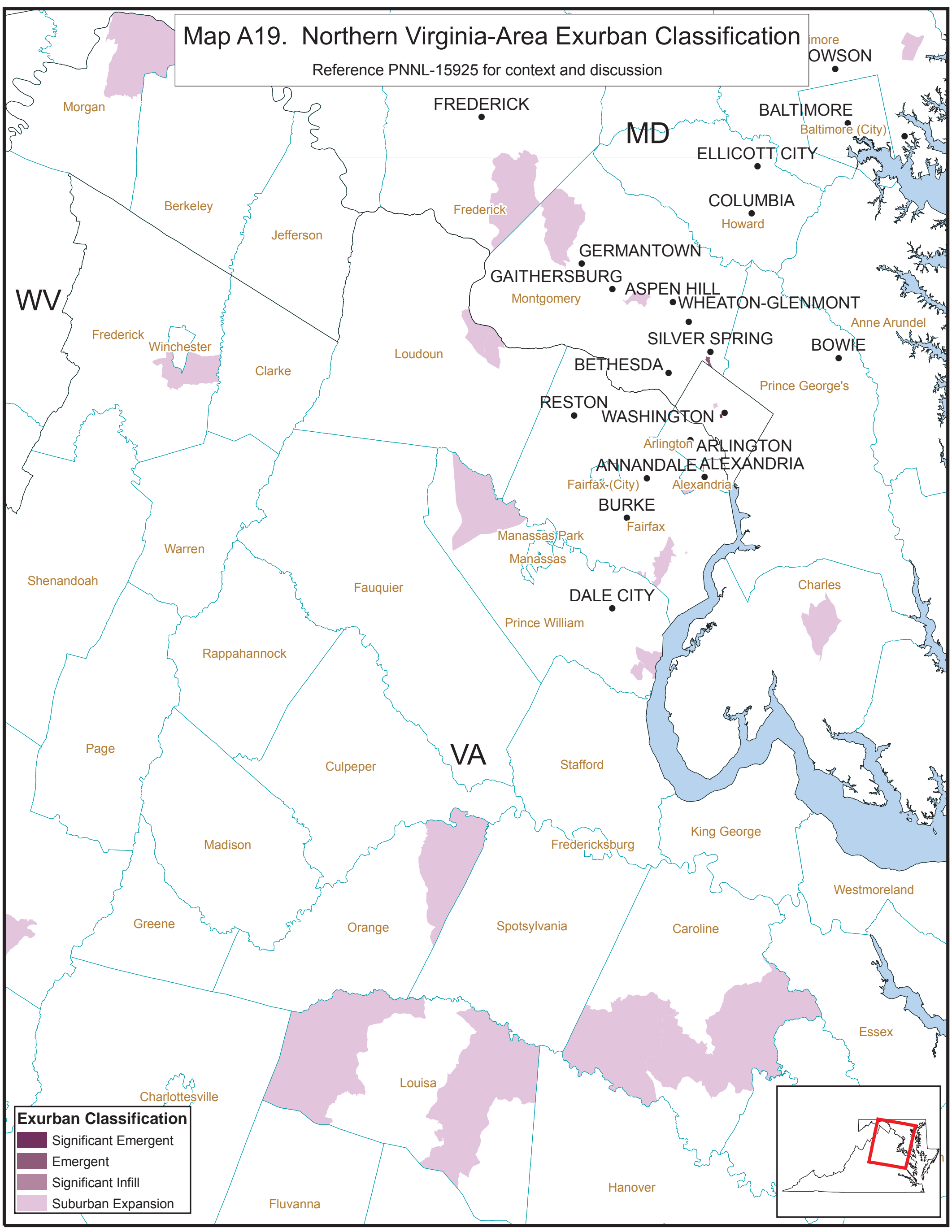




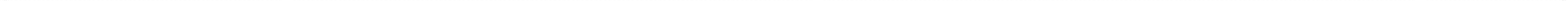




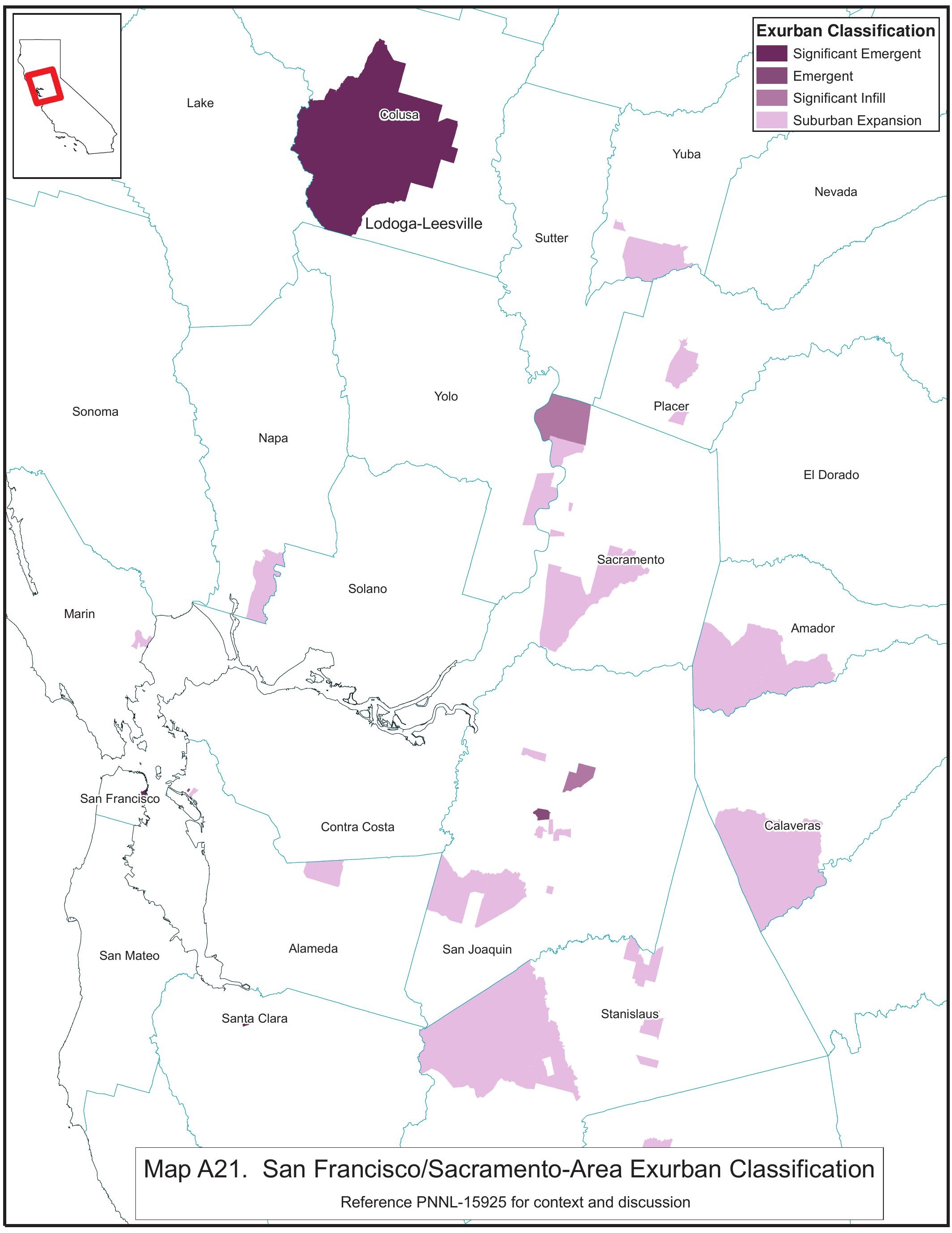









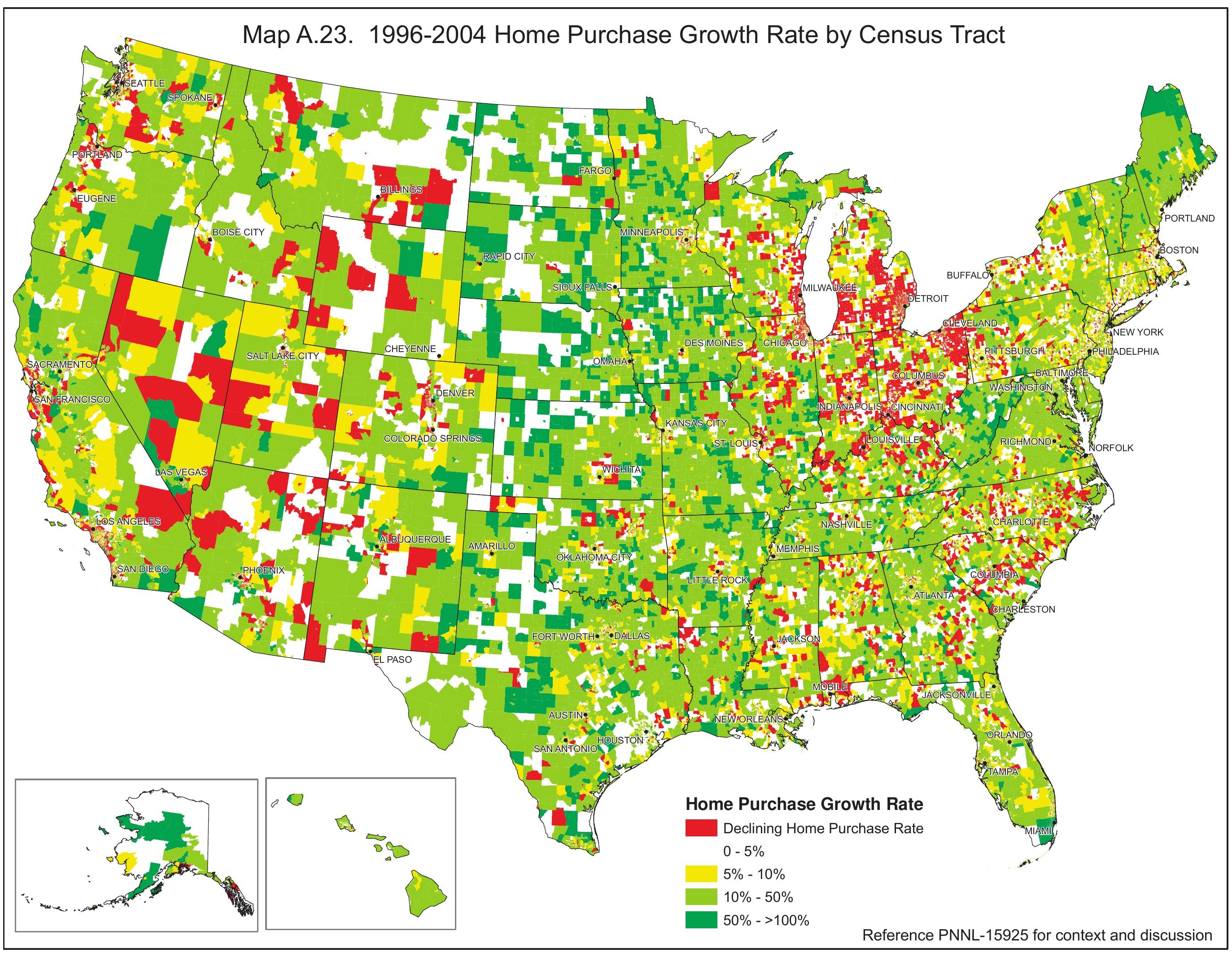




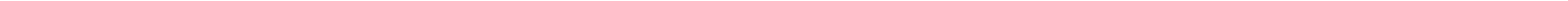









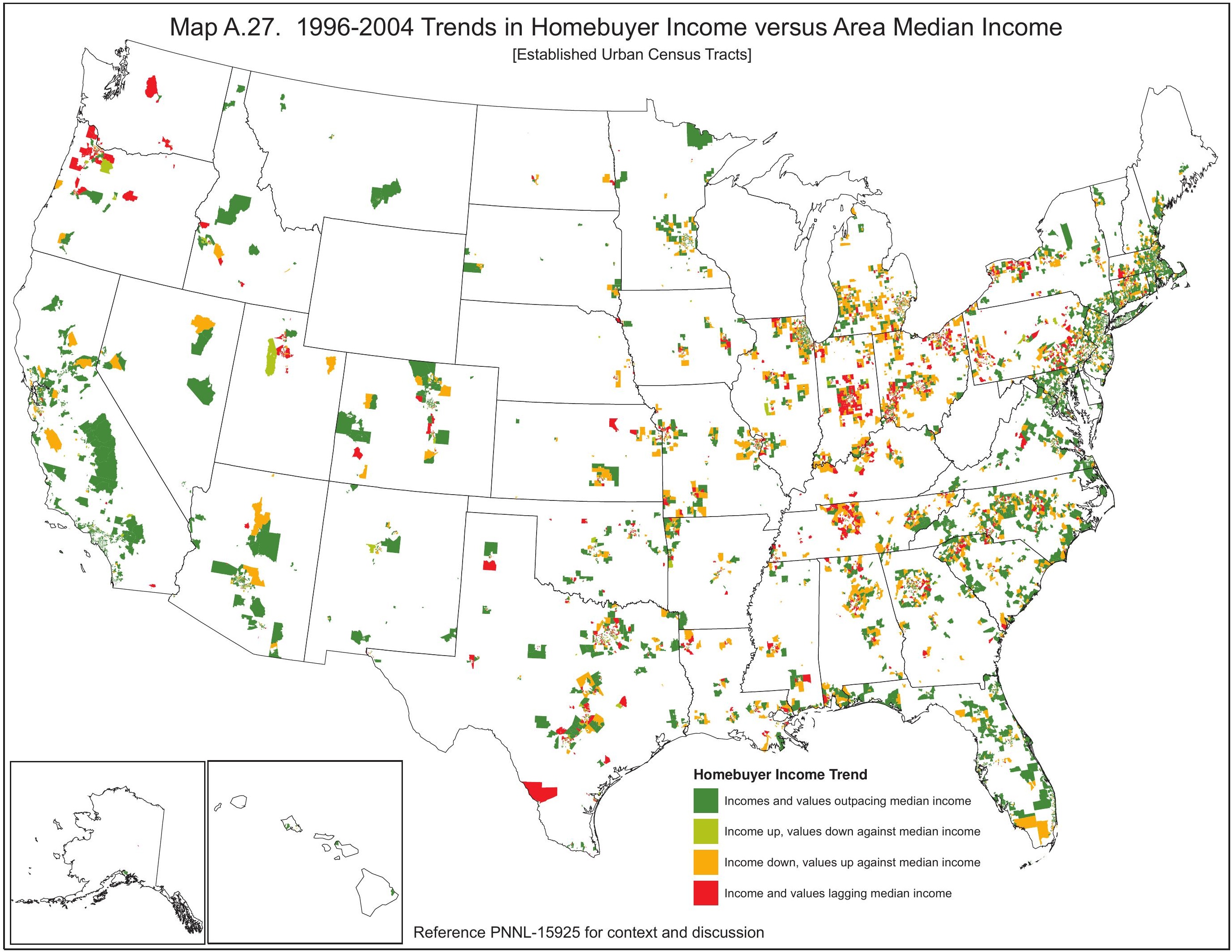


Map A.28. 1996-2004 Average Number of Home Purchases by Census Tract
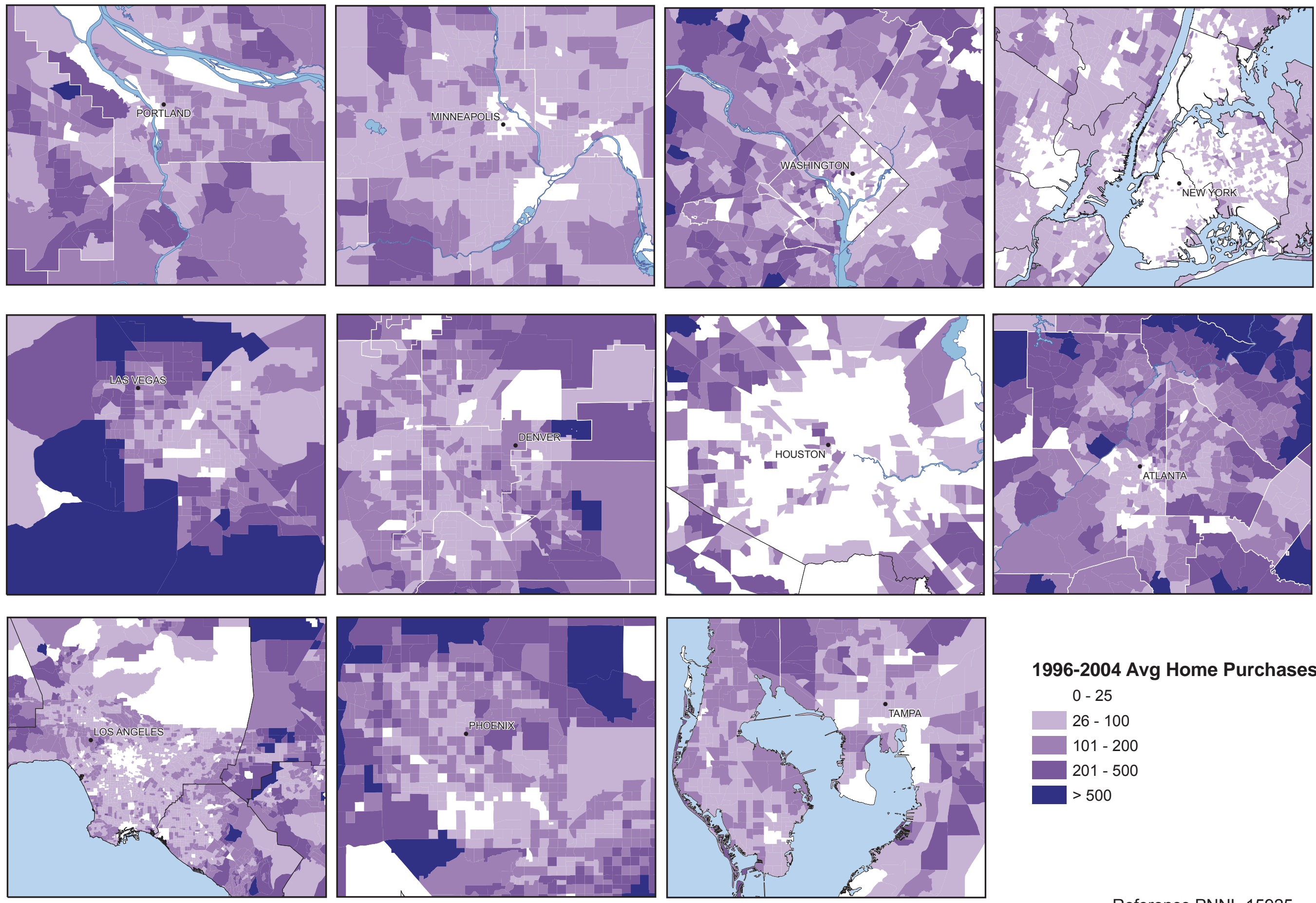

1996-2004 Avg Home Purchases $0-25$

$26-100$

$101-200$

$201-500$

$>500$ 
Map A.30. 1996-2004 Home Purchase Growth Rate by Census Tract
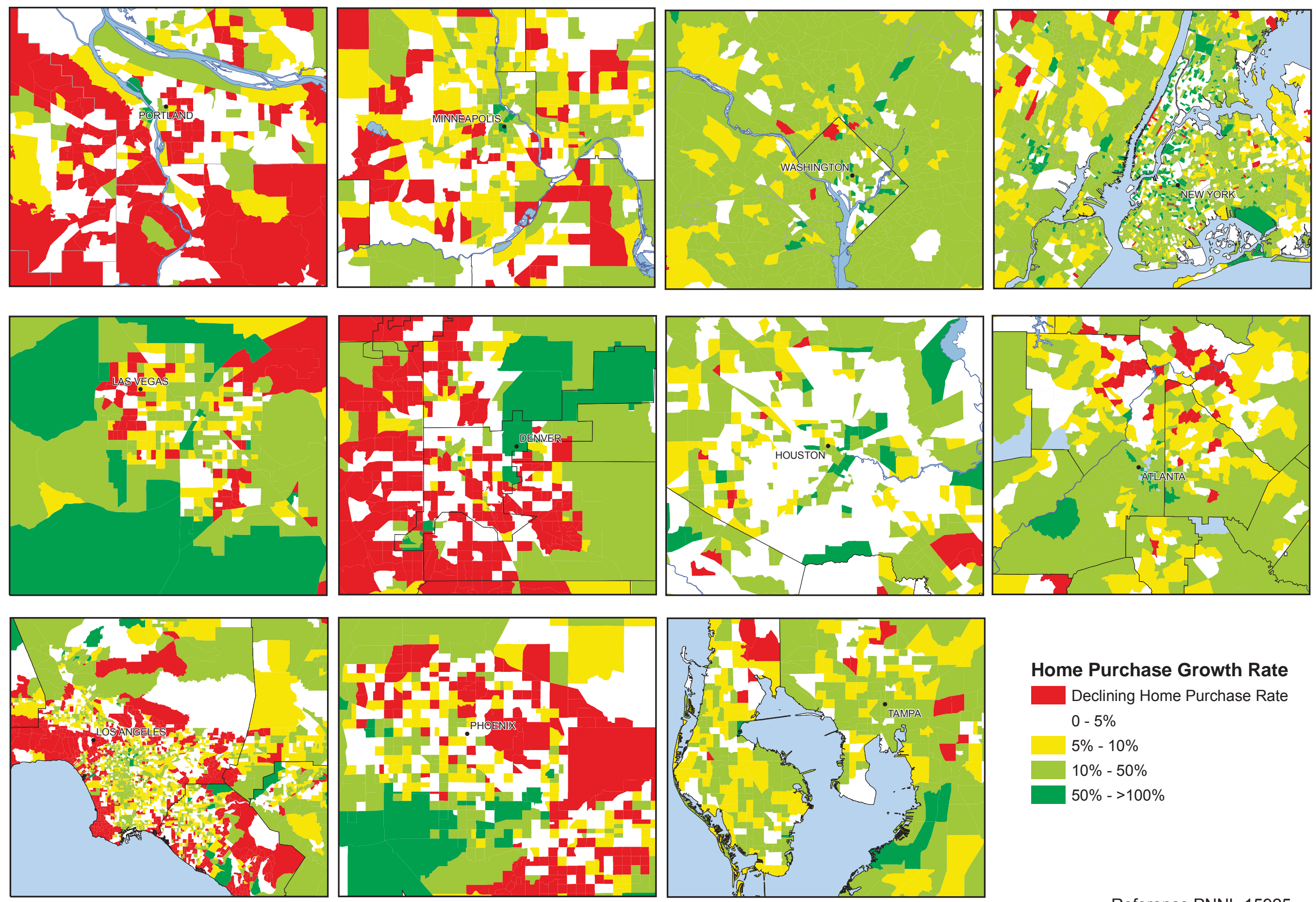

Home Purchase Growth Rate

Declining Home Purchase Rate $0-5 \%$

$5 \%-10 \%$

$10 \%-50 \%$

$50 \%->100 \%$ 


\section{Map A.32. 2004 Home Purchase Lender Market Share by Census Tract}
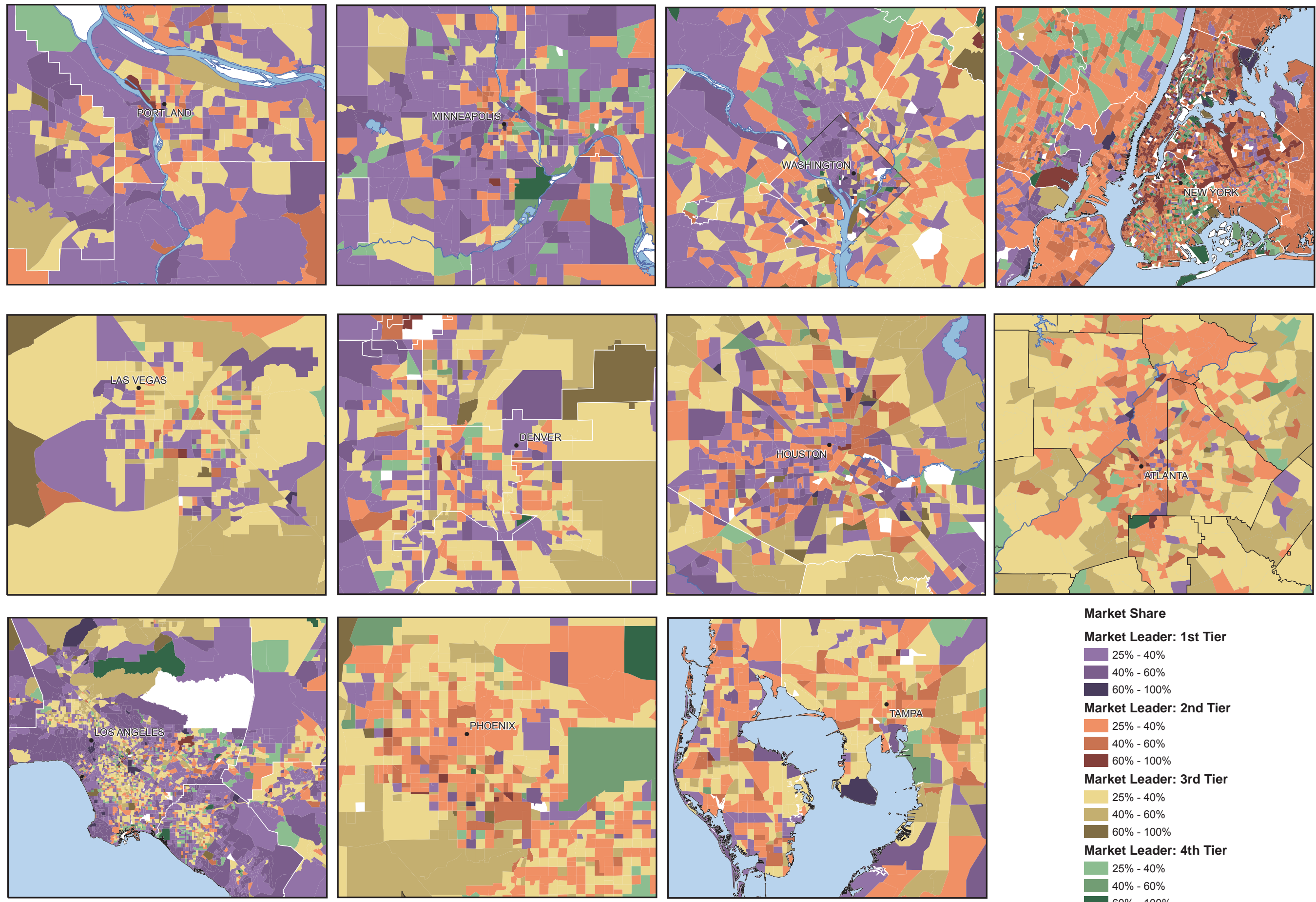

Market Share

Market Leader: 1st Tier

$25 \%-40 \%$

$40 \%-60 \%$

$60 \%-100 \%$

Market Leader: 2nd Tier

$25 \%-40 \%$

$40 \%-60 \%$

$60 \%-100 \%$

Market Leader: 3rd Tier

$25 \%-40 \%$

$40 \%-60 \%$

$60 \%-100 \%$

Market Leader: 4th Tier

$25 \%-40 \%$

$40 \%-60 \%$
$60 \%-100 \%$

Reference PNNL-15925 for context and discussion 